OBLIQUE SURFACE CRACKING AND CRACK CLOSURE IN AN ORTHOTROPIC MEDIUM UNDER CONTACT LOADING

A THESIS SUBMITTED TO

THE GRADUATE SCHOOL OF NATURAL AND APPLIED SCIENCES $\mathrm{OF}$

MIDDLE EAST TECHNICAL UNIVERSITY

BY

SELIM ERCİHAN TOKTAŞ

IN PARTIAL FULFILLMENT OF THE REQUIREMENTS

FOR

THE DEGREE OF MASTER OF SCIENCE

IN

MECHANICAL ENGINEERING

SEPTEMBER 2018 

Approval of the thesis:

\section{OBLIQUE SURFACE CRACKING AND CRACK CLOSURE IN AN ORTHOTROPIC MEDIUM UNDER CONTACT LOADING}

submitted by SELIM ERCIHAN TOKTAŞ in partial fulfillment of the requirements for the degree of Master of Science in Mechanical Engineering Department, Middle East Technical University by,

Prof. Dr. Halil Kalıpçılar

Dean, Graduate School of Natural and Applied Sciences

Prof. Dr. Mehmet Ali Sahir Arıkan

Head of Department, Mechanical Engineering

Prof. Dr. Serkan Dağ

Supervisor, Mechanical Engineering Dept., METU

\section{Examining Committee Members:}

Prof. Dr. Hakan Işı1k Tarman

Mechanical Engineering Dept., METU

Prof. Dr. Serkan Dağ

Mechanical Engineering Dept., METU

Asst. Prof. Dr. Hüsnü Dal

Mechanical Engineering Dept., METU

Asst. Prof. Dr. Sezer Özerinç

Mechanical Engineering Dept., METU

Asst. Prof. Dr. Tunç Apatay

Mechanical Engineering Dept., Gazi University

Date: 07.09.2018 
I hereby declare that all information in this document has been obtained and presented in accordance with academic rules and ethical conduct. I also declare that, as required by these rules and conduct, I have fully cited and referenced all material and results that are not original to this work.

Name, Last name : Selim Ercihan TOKTAŞ

Signature : 


\title{
ABSTRACT \\ OBLIQUE SURFACE CRACKING AND CRACK CLOSURE IN AN ORTHOTROPIC MEDIUM UNDER CONTACT LOADING
}

\author{
Toktaş, Selim Ercihan \\ M.S., Department of Mechanical Engineering \\ Supervisor: Prof. Dr. Serkan Dağ \\ September 2018, 123 pages
}

In this study, inclined surface cracks in an elastic orthotropic half-plane exposed to contact loading at the material surface are examined for both open and closed crack assumptions. At first, mixed mode stress intensity factors are computed for fully open crack assumption depending on the solution of singular integral equations, obtained by Full Fourier Transform techniques. Unless fully open crack assumption is valid, the suitable crack closure mode is determined. Both crack tip and full length closure modes are examined. Singular integral equations are solved with a suitable expansion-collocation technique depending on the closure mode. Finally, the closed portions of the cracks, contact pressure distributions between crack faces and modified mode II stress intensity factors are computed considering sliding crack face conditions. The main result of the analyses is the influence of the material parameters, the crack orientation angle and applied load on mixed mode stress intensity factors, closed portions of the cracks and the contact pressure distributions in the closed parts.

Keywords: Inclined Edge Crack, Stress Intensity Factors, Crack Closure Problems, Singular Integral Equations 


\title{
TEMAS YÜKLEMESİ ALTINDA ORTOTROPIKK ORTAMDAKİ EĞİK YÜZEY ÇATLAĞI VE ÇATLAK KAPANMASI
}

\author{
Toktaş, Selim Ercihan \\ Yüksek Lisans, Makine Mühendisliği Bölümü \\ Tez Yöneticisi: Prof. Dr. Serkan Dağ \\ Eylül 2018, 123 sayfa
}

$\mathrm{Bu}$ çalışmada, malzeme yüzeyinden temas yüklemesine maruz kalan elastik ortotropik yarı düzlemdeki eğik yüzey çatlakları, açık ve kapalı çatlak varsayımlarıyla incelenmiştir. İlk olarak karışı mod gerilme şiddet çarpanları tamamen açık çatlak varsayımıyla, Tam Fourier Dönüşümü teknikleriyle elde edilen tekil integral denklemlerinin çözümüne göre hesaplanmıştır. Tamamen açık çatlak varsayımı geçerli değilse, uygun olan çatlak kapanma modu tespit edilmiştir. Hem çatlak ucu hem de çatlağın tüm uzunluğunda oluşan kapanma modları incelenmiştir. Tekil integral denklemleri çatlak kapanma moduna bağlı olarak uygun bir siralama-düzenleme tekniğiyle çözülmüştür. Son olarak çatlakların kapanan bölümleri, çatlak yüzeyleri arasındaki temas gerilme dağılımları ve modifiye edilmiş ikinci mod gerilme şiddet çarpanları kayan yüzey şartlarına göre hesaplanmıştır. Analizlerin ana sonucu, malzeme parametrelerinin, çatlak açısının ve uygulanan yükün gerilme şiddet çarpanlarına, çatlakların kapanan kısımlarına ve çatlakların kapanan kısımlarındaki temas gerilme dağglımlarına olan tesiridir.

Anahtar Kelimeler: Eğik Yüzey Çatlağı, Gerilme Şiddet Çarpanları, Çatlak Kapanma Problemleri, Tekil İntegral Denklemleri. 
To my parents Zehra TOKTAŞ and Ali Kemal TOKTAŞ 


\section{ACKNOWLEDGMENTS}

The author owes a deep debt of gratitude to his supervisor Prof.Dr. Serkan DAĞ for his precious advices, collegiality and mentorship throughout this study. It would not have been possible to complete this thesis without the help, guidance and expertise of him and it was also a pleasure to work with him. 


\section{TABLE OF CONTENTS}

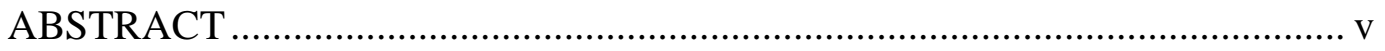

ÖZ

ACKNOWLEDGMENTS ............................................................................ vii

TABLE OF CONTENTS ………………………………............................. ix

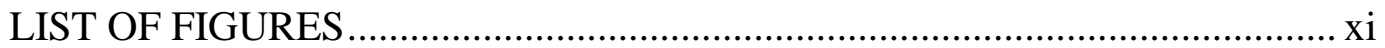

LIST OF SYMBOLS AND ABBREVIATIONS............................................. xix

\section{CHAPTERS}

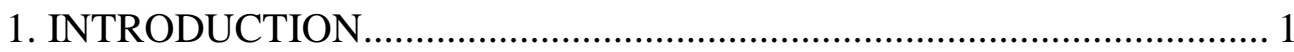

1.1 Literature Survey ………………………………………………….. 1

1.2 Scope of the Study ………………………………………………... 7

2. PROBLEM STATEMENT AND FORMULATION ………...................... 11

2.1 Problem Definition................................................................................ 11

2.2 Formulation of the Problem ................................................................ 11

2.3 The Contact Loading Problem ……………………………………... 16

2.4 Determination of Loading Function $\left(\sigma\left(x_{2}\right)\right)$................................ 20

2.4.1 Pressure Distribution for Flat Stamp..................................... 22

2.4.2 Pressure Distribution for Triangular Stamp ………………..... 25

2.4.3 Pressure Distribution for Circular Stamp................................ 26

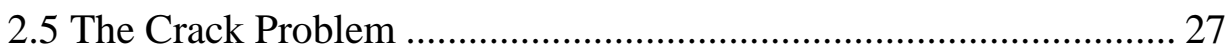

2.6 The Solution of Singular Integral Equations...................................... 41 
2.7 Implementation of Crack Closure 46

2.7.1 Full Length Closure Mode ................................................... 50

2.7.2 Crack Tip Closure Mode .................................................... 52

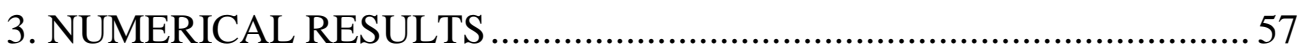

3.1 Figures for Orthotropic Half-Plane Indented by Flat Stamp (Fully

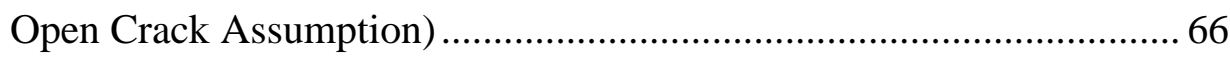

3.2 Figures for Orthotropic Half-Plane Indented by Triangular Stamp (Fully Open Crack Assumption) .......................................................... 77

3.3 Figures for Orthotropic Half-Plane Indented by Circular Stamp (Fully Open Crack Assumption) ........................................................ 88

3.4 Figures for Orthotropic Half-Plane Indented by Flat Stamp (Crack Tip and Full Closure Modes) ............................................................. 99

4. CONCLUSIONS AND FUTURE WORK …......................................... 107

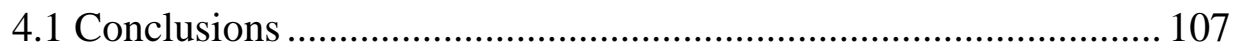

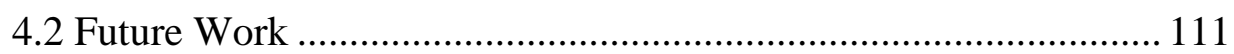

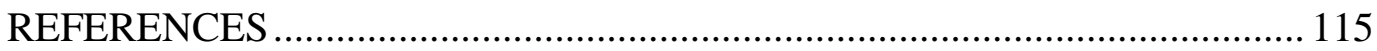

APPENDIX A. EXPLICIT RELATIONS OF THE KERNELS OF CONTACT

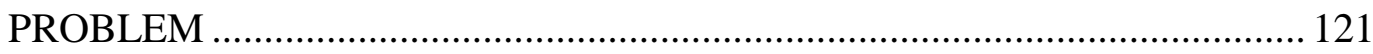

APPENDIX B. CLOSED FORM EXPRESSIONS FOR CAUCHY PRINCIPAL

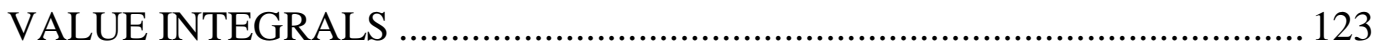




\section{LIST OF FIGURES}

Figure 2.1 The General Description of Problem ............................................... 12

Figure 2.2 Solution Procedure of the Coupled Problem .................................... 15

Figure 2.3 Solution Procedure of the Crack Problem ........................................ 16

Figure 2.4 Geometry of Contact Loading Problem........................................ 17

Figure 2.5 Types of Stamp Profiles................................................................. 22

Figure 2.6 Sample Pressure Distributions for Plane Stress Case, $\eta_{1}=0.6 \ldots \ldots \ldots .27$

Figure 2.7 Geometry of Crack Problem ........................................................ 28

Figure 2.8 Infinite Plane Problem with a Crack ............................................. 29

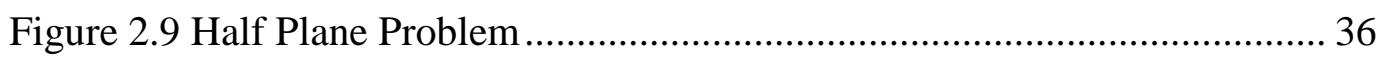

Figure 2.10 The Contour for Evaluation of Integral ....................................... 39

Figure 2.11 Crack Closure Mode Shapes ...................................................... 47

Figure 2.12 Sample Contact Pressure Distributions for Fully Closed Cracks

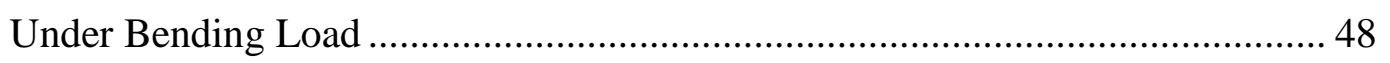

Figure 2.13 Sample Contact Pressure Distributions for Fully Closed Cracks Loaded by Punch Indentation........................................................................ 48

Figure 3.1 Representation of the Problem by Beghini et al [42]........................ 57

Figure 3.2 Comparison of SIF's for Inclined Edge Cracks in a) Isotropic Material

b) Orthotropic Material under Uniform Tension................................................ 59

Figure 3.3 Effect of Elastic Modulus Ratio $E_{2} / E_{1}$ on Mixed Mode Stress Intensity Factors for Inclined Edge Crack in Orthotropic Half-Plane under Uniform Tension for Plane Strain Case......................................................................... 59 
Figure 3.4 Comparison of Mixed Mode Stress Intensity Factors for Straight Edge Crack Provided by Sarikaya [2] (Markers) and Present Study (Lines) in Orthotropic Half-Plane of Plasma Sprayed Alumina Loaded by Flat Punch for Plane Strain Case and Fully Open Crack Assumption, $(b-a) / d=1$ 60

Figure 3.5 Comparison of Mixed Mode Stress Intensity Factors for Straight Edge Crack Provided by Sarikaya [2] (Markers) and Present Study (Lines) in Orthotropic Half-Plane of Plasma Sprayed Alumina Loaded by Triangular Punch for Plane Strain Case and Fully Open Crack Assumption, $(b-a) / d=1$. 61

Figure 3.6 Comparison of Mixed Mode Stress Intensity Factors for Straight Edge Crack Provided by Sarikaya [2] (Markers) and Present Study (Lines) in Orthotropic Half-Plane of Plasma Sprayed Alumina Loaded by Circular Punch for Plane Strain Case and Fully Open Crack Assumption, $(b-a) / d=1$. 61

Figure 3.7 Representation of the Problem by Beghini et al [43]. (Inclined Edge Crack under Bending Load) 62

Figure 3.8 Comparison of SIF's by Beghini et al [43] (Markers) and Present Study (Lines) for Inclined Edge Crack under Bending Load. (Orthotropic Material Constants: $\left.\mathrm{E}_{1}=116.37 \mathrm{GPa}, \mathrm{E}_{2}=116.35 \mathrm{GPa}, \mu_{12}=46.54 \mathrm{GPa}, \boldsymbol{v}=0.25\right) \ldots 64$ Figure 3.9 Comparison of Normal Contact Pressure Distributions by Beghini et al [43] (Markers) and Present Study (Lines) for Frictionless Contact. (Orthotropic Material Constants: $\left.\mathrm{E}_{1}=116.37 \mathrm{GPa}, \mathrm{E}_{2}=116.35 \mathrm{GPa}, \mu_{12}=46.54 \mathrm{GPa}, \boldsymbol{v}=0.25\right) \ldots 64$ Figure 3.10 Mixed Mode Stress Intensity Factors for Inclined Edge Crack in Orthotropic Half-Plane of Plasma Sprayed Alumina Loaded by Flat Punch for Plane Strain Case and Fully Open Crack Assumption, $(b-a) / d=1$, Crack Angle $\theta=\pi / 12$. 66

Figure 3.11 Mixed Mode Stress Intensity Factors for Inclined Edge Crack in Orthotropic Half-Plane of Plasma Sprayed Alumina Loaded by Flat Punch for Plane Strain Case and Fully Open Crack Assumption, $(b-a) / d=1, \eta_{1}=0.2 .67$ 
Figure 3.12 Mixed Mode Stress Intensity Factors for Inclined Edge Crack in Orthotropic Half-Plane of Plasma Sprayed Alumina Loaded by Flat Punch for Plane Strain Case and Fully Open Crack Assumption, $(b-a) / d=1, \eta_{1}=0.4 .68$

Figure 3.13 Effect of $(b-a) / d$ Variation on Mixed Mode Stress Intensity Factors for Inclined Edge Crack in Orthotropic Half-Plane of Plasma Sprayed Alumina Loaded by Flat Punch for Plane Strain Case and Fully Open Crack Assumption, Crack Angle $\theta=\pi / 12, \eta_{1}=0.2$.

Figure 3.14 Effect of $(b-a) / d$ Variation on Mixed Mode Stress Intensity Factors for Inclined Edge Crack in Orthotropic Half-Plane of Plasma Sprayed Alumina Loaded by Flat Punch for Plane Strain Case and Fully Open Crack Assumption, Crack Angle $\theta=\pi / 12, \eta_{1}=0.4$. 70

Figure 3.15 Effect of Elastic Modulus Ratio $E_{2} / E_{1}$ on Mixed Mode Stress Intensity Factors for Inclined Edge Crack in Orthotropic Half-Plane Loaded by Flat Punch for Plane Strain Case and Fully Open Crack Assumption, $(b-a) / d=$

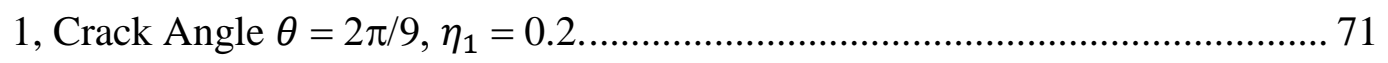

Figure 3.16 Effect of Elastic Modulus Ratio $E_{2} / E_{1}$ on Mixed Mode Stress Intensity Factors for Inclined Edge Crack in Orthotropic Half-Plane Loaded by Flat Punch for Plane Strain Case and Fully Open Crack Assumption, $(b-a) / d=$

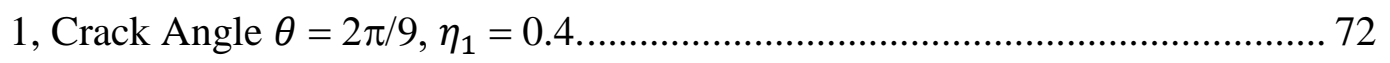

Figure 3.17 Effect of Elastic Modulus Ratio $E_{3} / E_{1}$ on Mixed Mode Stress Intensity Factors for Inclined Edge Crack in Orthotropic Half-Plane Loaded by Flat Punch for Plane Strain Case and Fully Open Crack Assumption, $(b-a) / d=$

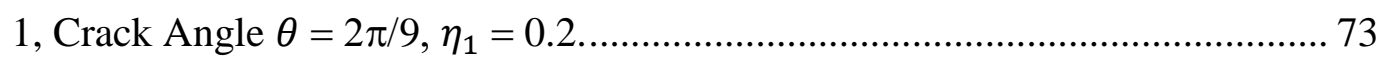

Figure 3.18 Effect of Elastic Modulus Ratio $E_{3} / E_{1}$ on Mixed Mode Stress Intensity Factors for Inclined Edge Crack in Orthotropic Half-Plane Loaded by Flat Punch for Plane Strain Case and Fully Open Crack Assumption, $(b-a) / d=$ 1 , Crack Angle $\theta=2 \pi / 9, \eta_{1}=0.4$. 74 
Figure 3.19 Effect of Crack Angle Variation on Mixed Mode Stress Intensity Factors for Inclined Edge Crack in Orthotropic Half-Plane of Plasma Sprayed Alumina Loaded by Flat Punch for Plane Strain Case and Fully Open Crack Assumption, $(b-a) / d=1, a / d=1$. 75

Figure 3.20 Effect of Crack Angle Variation on Mixed Mode Stress Intensity Factors for Inclined Edge Crack in Orthotropic Half-Plane of Plasma Sprayed Alumina Loaded by Flat Punch for Plane Strain Case and Fully Open Crack Assumption, $(b-a) / d=1, a / d=2$. .76

Figure 3.21 Mixed Mode Stress Intensity Factors for Inclined Edge Crack in Orthotropic Half-Plane of Plasma Sprayed Alumina Loaded by Triangular Punch for Plane Strain Case and Fully Open Crack Assumption, $(b-a) / d=1$, Crack Angle $\theta=\pi / 12$. .77

Figure 3.22 Mixed Mode Stress Intensity Factors for Inclined Edge Crack in Orthotropic Half-Plane of Plasma Sprayed Alumina Loaded by Triangular Punch for Plane Strain Case and Fully Open Crack Assumption, $(b-a) / d=1, \eta_{1}$ $=0.2$. 78

Figure 3.23 Mixed Mode Stress Intensity Factors for Inclined Edge Crack in Orthotropic Half-Plane of Plasma Sprayed Alumina Loaded by Triangular Punch for Plane Strain Case and Fully Open Crack Assumption, $(b-a) / d=1, \eta_{1}$ $=0.4$.

Figure 3.24 Effect of $(b-a) / d$ Variation on Mixed Mode Stress Intensity Factors for Inclined Edge Crack in Orthotropic Half-Plane of Plasma Sprayed Alumina Loaded by Triangular Punch for Plane Strain Case and Fully Open Crack Assumption, Crack Angle $\theta=\pi / 12, \eta_{1}=0.2$. 80

Figure 3.25 Effect of $(b-a) / d$ Variation on Mixed Mode Stress Intensity Factors for Inclined Edge Crack in Orthotropic Half-Plane of Plasma Sprayed Alumina Loaded by Triangular Punch for Plane Strain Case and Fully Open Crack Assumption, Crack Angle $\theta=\pi / 12, \eta_{1}=0.4$. 81 
Figure 3.26 Effect of Elastic Modulus Ratio $E_{2} / E_{1}$ on Mixed Mode Stress Intensity Factors for Inclined Edge Crack in Orthotropic Half-Plane Loaded by Triangular Punch for Plane Strain Case and Fully Open Crack Assumption, $(b-a) / d=1$, Crack Angle $\theta=2 \pi / 9, \eta_{1}=0.2$. 82

Figure 3.27 Effect of Elastic Modulus Ratio $E_{2} / E_{1}$ on Mixed Mode Stress Intensity Factors for Inclined Edge Crack in Orthotropic Half-Plane Loaded by Triangular Punch for Plane Strain Case and Fully Open Crack Assumption, $(b-a) / d=1$, Crack Angle $\theta=2 \pi / 9, \eta_{1}=0.4$.

Figure 3.28 Effect of Elastic Modulus Ratio $E_{3} / E_{1}$ on Mixed Mode Stress Intensity Factors for Inclined Edge Crack in Orthotropic Half-Plane Loaded by Triangular Punch for Plane Strain Case and Fully Open Crack Assumption, $(b-a) / d=1$, Crack Angle $\theta=2 \pi / 9, \eta_{1}=0.2$. 84

Figure 3.29 Effect of Elastic Modulus Ratio $E_{3} / E_{1}$ on Mixed Mode Stress Intensity Factors for Inclined Edge Crack in Orthotropic Half-Plane Loaded by Triangular Punch for Plane Strain Case and Fully Open Crack Assumption, $(b-a) / d=1$, Crack Angle $\theta=2 \pi / 9, \eta_{1}=0.4$. 85

Figure 3.30 Effect of Crack Angle Variation on Mixed Mode Stress Intensity Factors for Inclined Edge Crack in Orthotropic Half-Plane of Plasma Sprayed Alumina Loaded by Triangular Punch for Plane Strain Case and Fully Open Crack Assumption, $(b-a) / d=1, a / d=1$. 86

Figure 3.31 Effect of Crack Angle Variation on Mixed Mode Stress Intensity Factors for Inclined Edge Crack in Orthotropic Half-Plane of Plasma Sprayed Alumina Loaded by Triangular Punch for Plane Strain Case and Fully Open Crack Assumption, $(b-a) / d=1, a / d=2$. 87

Figure 3.32 Mixed Mode Stress Intensity Factors for Inclined Edge Crack in Orthotropic Half-Plane of Plasma Sprayed Alumina Loaded by Circular Punch for Plane Strain Case and Fully Open Crack Assumption, $(b-a) / d=1$, Crack Angle $\theta=\pi / 12$. 88 
Figure 3.33 Mixed Mode Stress Intensity Factors for Inclined Edge Crack in Orthotropic Half-Plane of Plasma Sprayed Alumina Loaded by Circular Punch for Plane Strain Case and Fully Open Crack Assumption, $(b-a) / d=1, \eta_{1}$ $=0.2$. 89

Figure 3.34 Mixed Mode Stress Intensity Factors for Inclined Edge Crack in Orthotropic Half-Plane of Plasma Sprayed Alumina Loaded by Circular Punch for Plane Strain Case and Fully Open Crack Assumption, $(b-a) / d=1, \eta_{1}$ $=0.4$

Figure 3.35 Effect of $(b-a) / d$ Variation on Mixed Mode Stress Intensity Factors for Inclined Edge Crack in Orthotropic Half-Plane of Plasma Sprayed Alumina Loaded by Circular Punch for Plane Strain Case and Fully Open Crack Assumption, Crack Angle $\theta=\pi / 12, \eta_{1}=0.2$.

Figure 3.36 Effect of $(b-a) / d$ Variation on Mixed Mode Stress Intensity Factors for Inclined Edge Crack in Orthotropic Half-Plane of Plasma Sprayed Alumina Loaded by Circular Punch for Plane Strain Case and Fully Open Crack Assumption, Crack Angle $\theta=\pi / 12, \eta_{1}=0.4$. 92

Figure 3.37 Effect of Elastic Modulus Ratio $E_{2} / E_{1}$ on Mixed Mode Stress Intensity Factors for Inclined Edge Crack in Orthotropic Half-Plane Loaded by Circular Punch for Plane Strain Case and Fully Open Crack Assumption, $(b-a) / d=1$, Crack Angle $\theta=2 \pi / 9, \eta_{1}=0.2$ 93

Figure 3.38 Effect of Elastic Modulus Ratio $E_{2} / E_{1}$ on Mixed Mode Stress Intensity Factors for Inclined Edge Crack in Orthotropic Half-Plane Loaded by Circular Punch for Plane Strain Case and Fully Open Crack Assumption, $(b-a) / d=1$, Crack Angle $\theta=2 \pi / 9, \eta_{1}=0.4$.

Figure 3.39 Effect of Elastic Modulus Ratio $E_{3} / E_{1}$ on Mixed Mode Stress Intensity Factors for Inclined Edge Crack in Orthotropic Half-Plane Loaded by Circular Punch for Plane Strain Case and Fully Open Crack Assumption, $(b-a) / d=1$, Crack Angle $\theta=2 \pi / 9, \eta_{1}=0.2$. 
Figure 3.40 Effect of Elastic Modulus Ratio $E_{3} / E_{1}$ on Mixed Mode Stress Intensity Factors for Inclined Edge Crack in Orthotropic Half-Plane Loaded by Circular Punch for Plane Strain Case and Fully Open Crack Assumption, $(b-a) / d=1$, Crack Angle $\theta=2 \pi / 9, \eta_{1}=0.4$. 96

Figure 3.41 Effect of Crack Angle Variation on Mixed Mode Stress Intensity Factors for Inclined Edge Crack in Orthotropic Half-Plane of Plasma Sprayed Alumina Loaded by Circular Punch for Plane Strain Case and Fully Open Crack Assumption, $(b-a) / d=1, a / d=1$.

Figure 3.42 Effect of Crack Angle Variation on Mixed Mode Stress Intensity Factors for Inclined Edge Crack in Orthotropic Half-Plane of Plasma Sprayed Alumina Loaded by Circular Punch for Plane Strain Case and Fully Open Crack Assumption, $(b-a) / d=1, a / d=2$. 98

Figure 3.43 Effect of $\eta_{2}$ on Mode II SIF for Inclined Edge Crack in Orthotropic Half-Plane of Plasma Sprayed Alumina Loaded by Flat Punch for Plane Strain Case, $(b-a) / d=1$, Crack Angle $\theta=\pi / 12$. 99

Figure 3.44 Effect of $\eta_{1}$ on Mode II SIF for Inclined Edge Crack in Orthotropic Half-Plane of Plasma Sprayed Alumina Loaded by Flat Punch for Plane Strain Case, $(b-a) / d=1$, Crack Angle $\theta=\pi / 18$ 100

Figure 3.45 a) Effect of Elastic Modulus Ratio $E_{2} / E_{1}$ for $\theta=2 \pi / 9$ b) Effect of Crack Angle Variation on Mode II SIF's for Inclined Edge Crack in Orthotropic Half-Plane Loaded by Flat Punch for Plane Strain Case, $(b-a) / d=1$. 101

Figure 3.46 a) Effect of $\eta_{2}$ on Mode II SIF for $(b-a) / d=0.1$, b) Effect of $a / d$ Variation on Contact Pressure Distributions Between Crack Faces for Inclined Edge Crack in Orthotropic Half-Plane of Plasma Sprayed Alumina Loaded by

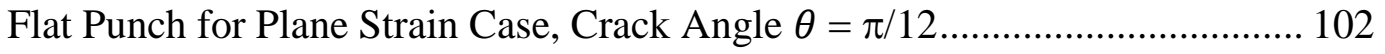

Figure 3.47 Effect of $a / d$ Variation on Contact Pressure Distributions Between Crack Faces for Straight Edge Crack in Orthotropic Half-Plane of Plasma Sprayed Alumina Loaded by Flat Punch for Plane Strain Case. 103 
Figure 3.48 Effect of Elastic Modulus Ratios $E_{2} / E_{1}$ and $E_{3} / E_{1}$ on Contact Pressure Distributions Between Crack Faces for Straight Edge Crack in Orthotropic HalfPlane Loaded by Flat Punch for Plane Strain Case, $a / d=1, \eta_{1}=0.2, \eta_{2}=0 \ldots 104$ Figure 3.49 Effect of $\eta_{2}$ on Normalized Relative Crack Surface Displacements for Inclined Edge Crack in Orthotropic Half-Plane of Plasma Sprayed Alumina Loaded by Flat Punch for Plane Strain Case, Crack Angle $\theta=\pi / 12, a / d=0.25$,

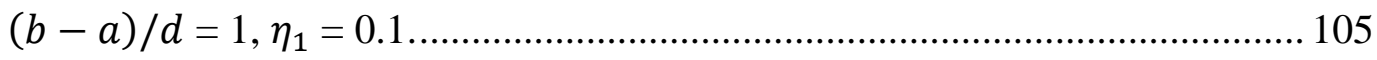

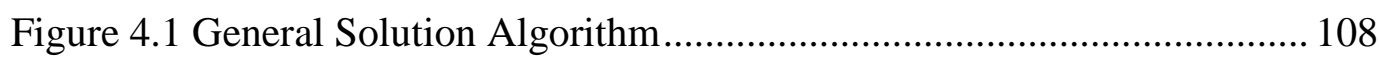

Figure 4.2 An Example of Other Closure Modes............................................ 112

Figure 4.3 Effect of $\eta_{2}$ on Sticking of Crack Faces for Inclined Edge Cracks under Bending Load for Plane Strain Case. (Mode I SIF's) 112

Figure 4.4 Effect of $\eta_{2}$ on Sticking of Crack Faces for Inclined Edge Cracks under Bending Load for Plane Strain Case. (Mode II SIF's) ............................. 113 


\section{LIST OF SYMBOLS AND ABBREVIATIONS}

$$
\begin{aligned}
& \sigma\left(x_{2}\right) \quad: \quad \text { Normal Loading Function } \\
& \tau\left(x_{2}\right) \quad \text { : Shear Loading Function } \\
& \boldsymbol{\theta} \quad \text { : Crack Angle } \\
& \text { d : Crack Length } \\
& \mathrm{CoF} \quad: \text { Coefficient of Friction } \\
& \eta_{1} \quad: \quad \text { CoF Between Material Surface and Loading Punch } \\
& \eta_{2} \quad: \text { CoF Between Crack Faces } \\
& u_{1}, u_{2}, U, V \quad: \quad \text { Displacement Components } \\
& f_{1}, f_{2} \quad: \text { Unknown Crack Surface Displacement Functions } \\
& \Phi_{1}, \Phi_{2} \quad \text { : Normalized Versions of } f_{1}, f_{2} \\
& \Phi \quad \text { : Normalized Version of Contact Loading Function } \\
& \psi_{1}, \psi_{2} \quad: \quad \text { Strength of Singularities at the End Points of the Contact } \\
& P_{n}^{\left(\psi_{2}, \psi_{1}\right)}, P_{k}^{\left(\psi_{2}, \psi_{1}\right)} \quad \text { : Jacobi Polynomials } \\
& \Gamma(\mathrm{l} \quad: \text { Gamma Function } \\
& h_{11}, h_{12}, h_{13} \quad: \text { Kernels of Singular Integral Equations } \\
& h_{21}, h_{22}, h_{23} \\
& \text { SIF } \quad: \text { Stress Intensity Factors } \\
& \text { SIE : Singular Integral Equations } \\
& \text { H( ) : Heaviside Step Function } \\
& e_{1}, e_{2} \quad: \quad \text { Closure Points }
\end{aligned}
$$




\section{CHAPTER 1}

\section{INTRODUCTION}

The main interest of this study is to evaluate SIF's for inclined edge cracks in orthotropic media with a solution method utilizing singular integral equations. A method is developed using integral transform techniques for an arbitrarily angled edge crack in orthotropic medium by considering the solution methods already available in the literature for different crack and material types. As discussed in the following sections, the conditions for fully open crack assumption cannot be satisfied for every loading case. The method in the present study is able to deal with the consequences of the crack closure on mixed mode stress intensity factors as well. In the following pages in Chapter 1, a summary about the studies in the literature related to this topic is given. Then, the scope of the study is specified.

\subsection{Literature Survey}

Conducted studies towards the investigation of fracture mechanics problems under various loading cases generally deal with isotropic materials. However, lastly developed materials in tribological applications need a comprehension of the behavior of nonisotropic material systems. Due to the demand of usage of different kind of orthotropic materials in tribological applications (some composite structures, coatings and thin films) over the years, there is an extensive volume of literature related on fracture mechanics of orthotropic materials. But, the lack of information on contact-related fracture of orthotropic surfaces is noticed in the literature. 
The calculation of SIF's for a closed crack is a non-linear problem in solid mechanics due to variable contact zone between the crack faces. The applied load and material parameters are the dominant factors for the determination of the closed part of the crack. For this reason, it is needed to apply different loads to work on all closure modes. Hence, related articles and dissertations concerning open or partially closed cracks under the effect of wide range of loads are briefly summarized in this section.

Several material type and loading cases for the coupled crack/contact problems are considered by Dag [1]. Crack/contact problems in isotropic medium and functionally graded isotropic medium are studied. Moreover, crack problems in general loading conditions are taken into consideration. It is observed that the singular behavior of the unknown crack functions are affected by the contact loading, if the contact loading area is very close to the crack. The main goal of the study is to investigate the mixed mode stress intensity factors at the crack tip and contact stress distribution at the material surface for sliding contact case. Numerical results are given for different combinations of friction coefficient, material nonhomogeneity parameter and crack/contact length parameter for flat, circular and triangular stamp profiles.

The study by Sarikaya [2] is related with the straight surface crack problem in an elastic orthotropic half-plane subjected to contact loading. The solution procedure developed only for crack problem in orthotropic strip by Delale and Erdogan [3] is adapted for the crack problem in orthotropic semi-infinite medium associated with the contact mechanics problem. Sample results are presented to detect the effect of the material properties and friction coefficient on the mixed mode stress intensity factors at the crack tip and the contact stresses.

Orthotropic materials related studies in fracture mechanics also provide stress intensity factors under different types of loading conditions by applying various methods. Li and Lee [4] work on a closed form solution for an orthotropic strip 
with a perpendicular internal crack under the effect of shear loading for four commonly encountered constraints. Ding and Zhou [5] deal with the interface cracks in a multi-layered orthotropic material under thermo-mechanical loading. Stress intensity factors evaluated by analytical and computational approaches are also available in the literature for functionally graded orthotropic materials [6],[7]. The enriched finite elements, specially located at the crack tip, enable the direct calculation of mixed mode stress intensity factors from the solution of a system of linear equations without any further process. In the study by Dag et al [8], a numerical solution based on the enriched finite elements is formulated for mixed mode fracture analysis of orthotropic functionally graded materials subjected to thermal stresses.

The general concepts about indentation theory of orthotropic materials loaded by a moving frictional punch are reported by Zhou et al [9]. In another study by Guler [10], a closed-form solution is given for two-dimensional frictional contact problem of an orthotropic medium. Dag et al [11] focus on evaluating Hertzian contact pressure distribution beneath a rigid circular stamp by both analytical and numerical procedures for orthotropic functionally graded materials.

The study by Dag et al [12] is an extension work of Dag [1]. The ideas have been improved by adding the effect of crack tip closure to the problem. The main emphasis is that partial closure of the crack faces takes place for small values of the friction coefficient at the material surface. The surfaces of the crack are assumed to be in frictionless contact for the closed portion of the crack. In the end, a boundary value problem, highly nonlinear in terms of the unknown length of the closed portion of the crack, is obtained. The length of the closed portion of the crack and the modified mode II stress intensity factors are evaluated with an iterative method.

Beghini et al [13] examine the closure conditions of oblique edge cracks under travelling loading case. The weight functions method, an analytical expression of 
the Green functions, is used to obtain the crack opening displacements. The sticking and sliding conditions of partial crack closure are studied using the crack opening displacements. The effects of different friction conditions in the closure region on mixed mode stress intensity factors during a typical loading cycle were investigated.

Birinci and Cakiroglu [14] consider mode I problem of an internal crack parallel to its surfaces in an infinite elastic isotropic layer. The system is loaded by a pair of compressive concentrated forces and a pair of uniform compressive stress along the crack surface. A normalized critical load factor, which starts the crack closure, is calculated. It is proved that the stress intensity factor reaches to its maximum value as the closure length approaches to zero. However, for fully closed cracks, it is zero which is an expected result because of the disappearance of the crack. The results show that the increase in the closure length decreases the stress intensity factor and prevents the crack propagation. Similar calculations are done by Kahya et al [15] for monoclinic, orthotropic and transversely isotropic materials.

The scope of the study by Hills and Nowell [16] is to examine the plane cracks under the effect of bulk stress field. At least some parts of the crack faces are pressed together due to this stress field. This situation is mostly faced in problems triggered by a contact stress field. However, the illustrative problems for simpler cases are given for normal and slant cracks. In this study, the distributed dislocation technique by Hills et al [17] is applied. This technique can be used in a wide range of solid mechanics problems including fretting, rolling contact fatigue, etc.

Porter and Hills [18] examine the problem of finding the mode II stress intensity factor for only fully closed inclined edge crack under the effect of a compressive stress field. The strategy is based on Bueckner's Superposition Principle. This is a corrective solution procedure. First, stress state in the uncracked body is found. 
Then, dislocations are revealed to simulate the existence of the crack. Accurate results are obtained for shallow cracks with friction present on the crack faces. The results show that friction on the crack faces reduces mode II stress intensity factor. This reduction of the shear stress by friction between the crack faces is strong enough to make $\mathrm{K}_{\mathrm{II}}$ equal to zero for low inclinations. Therefore, sticking may occur for shallow cracks.

Choi [19] conducts parametric studies about two dissimilar half-planes bonded to each other with a graded interfacial zone containing an inclined crack. For external loading conditions, the uniform crack surface tractions and the remote biaxial loading cases are considered separately. The estimation of probable cleavage angles of the crack growth direction is done. Also, some hints about how crack closure occurs are given.

Guo et al [20] investigate the arbitrarily oriented interface crossing crack problem between two graded layers. The results prove that the mixed-mode SIF's are extremely affected by the crack angle. It is evidence that the oblique crack problems should be examined in more details. In other words, the study indicates that the consideration of merely horizontal and vertical cracks is not enough. In most cases, the maximum levels for SIF's are obtained when the crack is on the intersection of two layers. In addition, this study gives the basic principles for establishing a multi-layered model for the arbitrarily oriented crack problem.

Delale et al [21] describe an orthotropic strip problem with an inclined crack. The crack is placed straight on an axis of orthotropy. However, the strip has a different angular orientation compared to the orthogonal axes of material orthotropy so that an inclined crack problem is obtained. The numerical results for mixed mode stress intensity factors are given for both embedded and edge cracks. 
An arbitrarily oriented crack problem in a FGM layer is examined in the study by Long and Delale [22]. Then, this problem is combined with a homogeneous halfplane problem by the same authors ( Long and Delale [23] ). The system is externally loaded by crack surface tractions. It is determined that as the crack angle increases, $K_{I}$ decreases all the time while $K_{I I}$ increases until some point and then it also decreases. Mode I fracture generally is dominant at the crack tips. Lastly, the presence of homogeneous substrate has negligible effects on stress intensity factors for small cracks away from the interface.

Multiple crack problems in nonhomogeneous orthotropic medium subjected to inplane loading is investigated by Monfared and Ayatollahi [24]. Combinations of circular arc crack and straight crack arrangements are examined in details. Jin and Keer [25] solve two types of multiple edge cracks problems in isotropic medium. A half-plane containing up to 100 oblique edge cracks case and periodical oblique edge cracks case are examined. Yildirim et al [26] also deal with repeatedly located cracks in FGMs. A unit cell approach is taken into consideration using periodicity. Then, the unit cell is treated as though it is a strip. In all three studies, the results are compared with the finite element implementations for verification.

Thermal stress intensity factors for a circumferential crack problem in a thin walled cylinder made of FGMs are calculated by Dag [27]. Thermomechanical properties of FGMs alter as exponential functions in the thickness direction. The thin walled cylinder problem is reduced to a plate problem using the axisymmetry. The fluctuation of thermal stress intensity factors is investigated by changing the thermomechanical properties of the material.

El-Borgi et al [28] and Dag et al [29] work on surface crack problem in graded coatings bonded to a homogeneous substrate. El-Borgi et al [28] choose to examine this problem under general loading conditions. On the other hand, the solution is done with a two-stage method for the sliding contact case by Dag et al 
[29]. First, only the sliding frictional contact problem with a rigid punch is solved for the contact stresses. Afterwards, these stresses are accepted as inputs for the crack problem. This is an uncoupled solution. Because, the influence of the presence of the crack on the contact stress distribution is ignored. However, the solution still gives accurate results in certain cases.

Guo et al [30] answer the problem of which direction that the cracks will propagate, for multiple embedded cracks in a strip by using the maximum circumferential stress criterion. It states that the crack will propagate from its tip with a critical cracking angle $\left(\theta_{c}\right)$, determined by using the fact that the circumferential stress in the direction of crack propagation is a principal stress. It is also found that if the distance between two cracks is ten times larger than the half length of the cracks, the existence of multiple cracks can be ignored. Also, the angular variations of the other cracks have inconsiderable effects on the critical cracking angle.

In fretting problems, it is a common knowledge that at the first step, a slant crack appears at the surface of the material. Then, this slant crack becomes rather normal with crack propagation. In other words, two or more straight crack segments with different crack angles exist in the material. Therefore, the resulting geometry will be a kinked crack. This situation is examined by Yingzhi and Hills [31] for a crack that remains open throughout its length. Variations of mixed mode stress intensity factors for the crack segments with different crack sizes and crack angles are given.

\subsection{Scope of the Study}

In certain engineering applications, the contact of two machine elements may be encountered, which leads to surface crack formation and propagation near the contact zone. In other words, contact between machine components is one of the origins of surface crack initiation. Indentation fractures ( Lawn [32] ) and fretting 
fractures ( Hills and Nowell [33] ) are the examples of these types of failures. The surface cracks triggered by fretting fatigue, are within the scope of this study.

The structures exposed to fretting fatigue experience with severe loading conditions caused by relative and repeated movement. This recurrent movement in fretting fatigue induces a significant decrease in the fatigue strength of the material. It also deteriorates the material surface. Hence, surface cracks initiate in the long run. It is observed that at first, a number of micro-cracks appear at the edge of the contact region, and then some of them merge in to one. Thus, the main crack becomes oblique to the contact surface in the early stage of crack growth in fretting fatigue problems. Kimura and Sato [34] state that the reduction of life in fretting fatigue is related with the early crack initiation and propagation in the short crack region. Therefore, a detailed investigation of the mixed mode stress intensity factors for an inclined edge crack is essential to be able to predict subcritical crack growth. This prediction is vital for safety and economic reasons in aircraft structures, bearings, engines, and so-forth.

In addition, Elber [35] observed that the crack face closure occurs in some loading conditions. This crucial phenomenon in fracture mechanics may substantially alter the results for mixed mode stress intensity factors. Therefore, the scope for this study is to examine the effects of crack angle and crack closure phenomenon for an inclined edge crack in the orthotropic half-plane under the effect of contact loading conditions.

As it is mentioned above, a model for contact-based fracture in an orthotropic material is developed by Sarikaya [2] for straight and fully open cracks. Present study provides compulsory and crucial extensions over the model developed by Sarikaya [2]. The model is enhanced by the consideration of crack orientation angle and modification of the solution with crack closure. Thus, crack growth behavior is taken into account in a more precise way for more realistic cracks. 
Therefore, the present study submits a procedure that embodies both crack angle and crack closure modes into the solution.

In Chapter 2, the definition of problem is given. The boundary conditions in the problem are identified. A brief overview about plane elasticity of orthotropic materials is mentioned. Then, the formulation of the problem is expressed. Overall problem is divided into 3 sub problems for simplification. These sub problems are considered by using Fourier Transform for the suitable variable. When the indentation of punches is selected as the load, the contact pressure distribution on the material surface is obtained by solving the sliding contact problem separately. Then, the stress field in the loading region of two bodies becomes a known function for the crack problem. The displacement and the stress fields are obtained in the integral form. Using the equilibrium equations, the overall problem is degraded to the system of one or two singular integral equations depending on the closure mode. In Chapter 3, several figures for the mixed mode stress intensity factors for both open and closed cracks and contact pressure distributions between crack faces are presented by changing material properties, crack angle and load type. Finally, concluding remarks and future work of this study are stated in Chapter 4. 


\section{CHAPTER 2}

\section{PROBLEM STATEMENT AND FORMULATION}

\subsection{Problem Definition}

The configuration of the main problem is illustrated in Figure 2.1. An orthotropic elastic half-plane is loaded by known functions $\sigma\left(x_{2}\right)$ and $\tau\left(x_{2}\right)$ as a representation of the contact of two solids. These loads are applied from $x_{2}=a$ to $x_{2}=b$ at the material surface and the half-plane contains an inclined edge crack of length $d$ and crack angle $\theta$. Clockwise direction is selected as positive direction for crack angle. Dag [1] showed that depending on the surface loads, negative values for mode I stress intensity factors may be obtained at the crack tip. The physical meaning of these results is that the surfaces of the crack must come into contact and take the shape of a cusp. Hence, at least some part of the crack is closed. However, this unknown closed portion of the crack is not designated until Section 2.7. Thus, the formulation continues with fully open crack assumption (the closed portion of the crack is assumed to be zero) until Section 2.7. In the formulation of the problem, two coordinate systems ( $X-Y$ and $\left.x_{1}-x_{2}\right)$ are introduced. The stress transformation between these coordinate systems can be applied whenever desired. Lastly, $E_{1}, E_{2}, \mu_{12}$ and $v_{i j}$ represent the Young's moduli, shear modulus, and Poisson's ratio of the material, respectively.

\subsection{Formulation of the Problem}

First of all, the mixed boundary conditions, needed to be verified, are determined. At the material surface, there are no normal and shear stresses except for the ones 
$\left(\sigma\left(x_{2}\right)\right.$ and $\left.\tau\left(x_{2}\right)\right)$ between $x_{2}=a$ and $x_{2}=b$. Coulomb's friction law is valid for shear stress in the loading area. The crack angle can be selected between -90 and 90 degrees. At the crack plane $(Y=0)$, tangential and normal displacements are nonzero for the inside of the crack $(X<d)$. Crack faces are stress free for the open portion of the crack. In addition, it is compulsory to be bounded for all field quantities (stresses and displacements) in the half-plane as $\left(X^{2}+Y^{2}\right)$ or $\left(x_{1}^{2}+x_{2}^{2}\right) \rightarrow \infty$. Note that the crack is loaded from the surface of the material as illustrated in Figure 2.1. Yet, these loads can be replaced by any type of loading as discussed in Section 2.7 and Chapter 3 for the verification of the present study with the results in the literature.

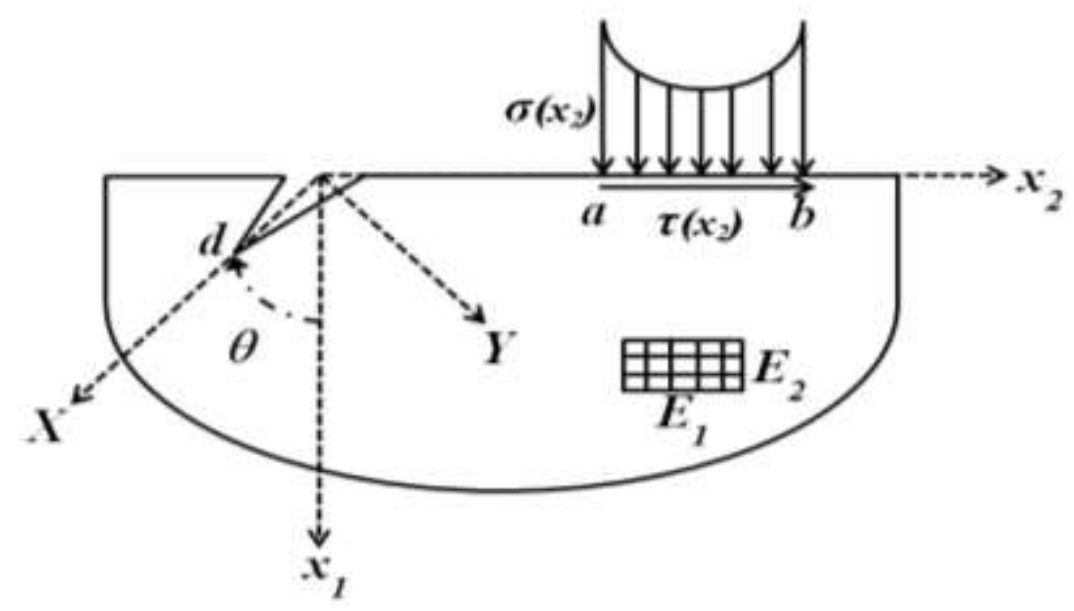

Figure 2.1 The General Description of Problem

To sum up, the main boundary conditions can be written as follows:

$$
\begin{array}{ll}
\sigma_{x_{1} x_{1}}\left(x_{1}=0, x_{2}\right)=0, & x_{2}<a \text { and } x_{2}>b \\
\sigma_{x_{1} x_{2}}\left(x_{1}=0, x_{2}\right)=0, & x_{2}<a \text { and } x_{2}>b
\end{array}
$$




$$
\begin{array}{lc}
\sigma_{x_{1} x_{1}}\left(x_{1}=0, x_{2}\right)=\sigma\left(x_{2}\right), & a<x_{2}<b \\
\sigma_{x_{1} x_{2}}\left(x_{1}=0, x_{2}\right)=\tau\left(x_{2}\right), & a<x_{2}<b \\
\tau\left(x_{2}\right)=\eta_{1} \sigma\left(x_{2}\right), & a<x_{2}<b \\
\sigma_{Y Y}(X, Y=0)=0, & 0<X<d \\
\sigma_{X Y}(X, Y=0)=0, & 0<X<d \\
\sigma_{x_{1} x_{1}}, \sigma_{x_{1} x_{2}}, \sigma_{x_{2} x_{2}} \rightarrow \text { bounded as } & \\
\sigma_{X X}, \sigma_{X Y}, \sigma_{Y Y} \rightarrow \text { bounded as } & \left(x_{1}^{2}+x_{2}^{2}\right) \rightarrow \infty \\
u_{1}\left(x_{1}, x_{2}\right), u_{2}\left(x_{1}, x_{2}\right) \rightarrow \text { bounded as } & \left(X^{2}+Y^{2}\right) \rightarrow \infty \\
U(X, Y), V(X, Y) \rightarrow \text { bounded as } &
\end{array}
$$

where $\eta_{1}$ in (2.1e) is the coefficient of friction. Note that, selected coordinate system is specified in the subscript. For instance, $\sigma_{x_{1} x_{1}}$ represents the normal stress in $x_{1}$ direction in $x_{1}-x_{2}$ coordinate system while $\sigma_{X Y}$ represents the shear stress in $X-Y$ coordinate system. The derivatives of crack surface displacements are used in the representation of the crack. In this way, the crack is defined with two unknown functions $f_{1}(X)$ and $f_{2}(X)$.

$$
\begin{array}{ll}
\frac{Q_{66}}{2} \frac{\partial}{\partial X}\left(V\left(X, 0^{+}\right)-V\left(X, 0^{-}\right)\right)=f_{1}(X) & 0<X<d \\
\frac{Q_{66}}{2} \frac{\partial}{\partial X}\left(U\left(X, 0^{+}\right)-U\left(X, 0^{-}\right)\right)=f_{2}(X), & 0<X<d
\end{array}
$$

where $Q_{66}$ is a material parameter. $V$ in (2.2a) and $U$ in (2.2b) represent the displacement components in $Y$ and $X$ directions, respectively. By neglecting the body forces, the equilibrium equations can be expressed as the following fashion.

$\frac{\partial \sigma_{x_{1} x_{1}}}{\partial x_{1}}+\frac{\partial \sigma_{x_{1} x_{2}}}{\partial x_{2}}=0 \quad$ or $\quad \frac{\partial \sigma_{X X}}{\partial X}+\frac{\partial \sigma_{X Y}}{\partial Y}=0$ 
$\frac{\partial \sigma_{x_{1} x_{2}}}{\partial x_{1}}+\frac{\partial \sigma_{x_{2} x_{2}}}{\partial x_{2}}=0 \quad$ or $\quad \frac{\partial \sigma_{X Y}}{\partial X}+\frac{\partial \sigma_{Y Y}}{\partial Y}=0$

The constitutive relations of orthotropic materials can be represented for plane stress and plane strain cases in the following form ( Sarikaya [2] ).

$\left[\begin{array}{l}\sigma_{11} \\ \sigma_{22} \\ \sigma_{12}\end{array}\right]=\left[\begin{array}{ccc}C_{11} & C_{12} & 0 \\ C_{12} & C_{22} & 0 \\ 0 & 0 & C_{66}\end{array}\right]\left[\begin{array}{l}\varepsilon_{11} \\ \varepsilon_{22} \\ \varepsilon_{12}\end{array}\right]$

$C_{11}, C_{12}, C_{22}$ and $C_{66}$ are stiffness terms and can be written in terms of material parameters as the equation set below.

$C_{11}= \begin{cases}\frac{E_{1}^{2}}{E_{1}-v_{12}^{2} E_{2}} & \text { Plane Stress } \\ \frac{\left(1-v_{23} v_{32}\right) E_{1}^{2}}{\Delta} & \text { Plane Strain }\end{cases}$
$C_{12}= \begin{cases}\frac{v_{12} E_{1} E_{2}}{E_{1}-v_{12}^{2} E_{2}} & \text { Plane Stress } \\ \frac{\left(v_{12}+v_{13} v_{32}\right) E_{1} E_{2}}{\Delta} & \text { Plane Strain }\end{cases}$
$C_{22}= \begin{cases}\frac{E_{1} E_{2}}{E_{1}-v_{12}^{2} E_{2}} & \text { Plane Stress } \\ \frac{\left(1-v_{31} v_{13}\right) E_{1} E_{2}}{\Delta} & \text { Plane Strain }\end{cases}$

$\begin{array}{ll}C_{66}=2 \mu_{12} & \text { For Both Cases }\end{array}$

$\Delta=E_{1} T_{1}-E_{2} T_{2}$

$T_{1}=\left(1-v_{23} v_{32}-v_{31} v_{13}+v_{31} v_{13} v_{23} v_{32}\right)$

$T_{2}=\left(v_{12}^{2}+2 v_{12} v_{13} v_{32}+v_{13}^{2} v_{32}^{2}\right)$

Now, the overall problem is divided into sub problems. First, crack and contact loading problems are separated. Then, crack problem is split into two as the crack problem in an infinite plane and the half-plane problem that satisfies the 
boundary conditions at the free surface. Thus, contact loading problem, infinite plane problem and half-plane problem are superposed to fulfill the requirements of the original problem. These subdivision methods are shown in Figure 2.2 and Figure 2.3.

The governing partial differential equations of the half-plane are written in the formulas below by utilizing equilibrium equations and constitutive relations of orthotropic materials.

$$
\begin{aligned}
& C_{11} \frac{\partial^{2} u_{1}}{\partial x_{1}{ }^{2}}+\frac{C_{66}}{2} \frac{\partial^{2} u_{1}}{\partial x_{2}{ }^{2}}+\left(C_{12}+\frac{C_{66}}{2}\right) \frac{\partial^{2} u_{2}}{\partial x_{1} \partial x_{2}}=0 \\
& \left(C_{12}+\frac{C_{66}}{2}\right) \frac{\partial^{2} u_{1}}{\partial x_{1} \partial x_{2}}+\frac{C_{66}}{2} \frac{\partial^{2} u_{2}}{\partial x_{1}{ }^{2}}+C_{22} \frac{\partial^{2} u_{2}}{\partial x_{2}{ }^{2}}=0
\end{aligned}
$$
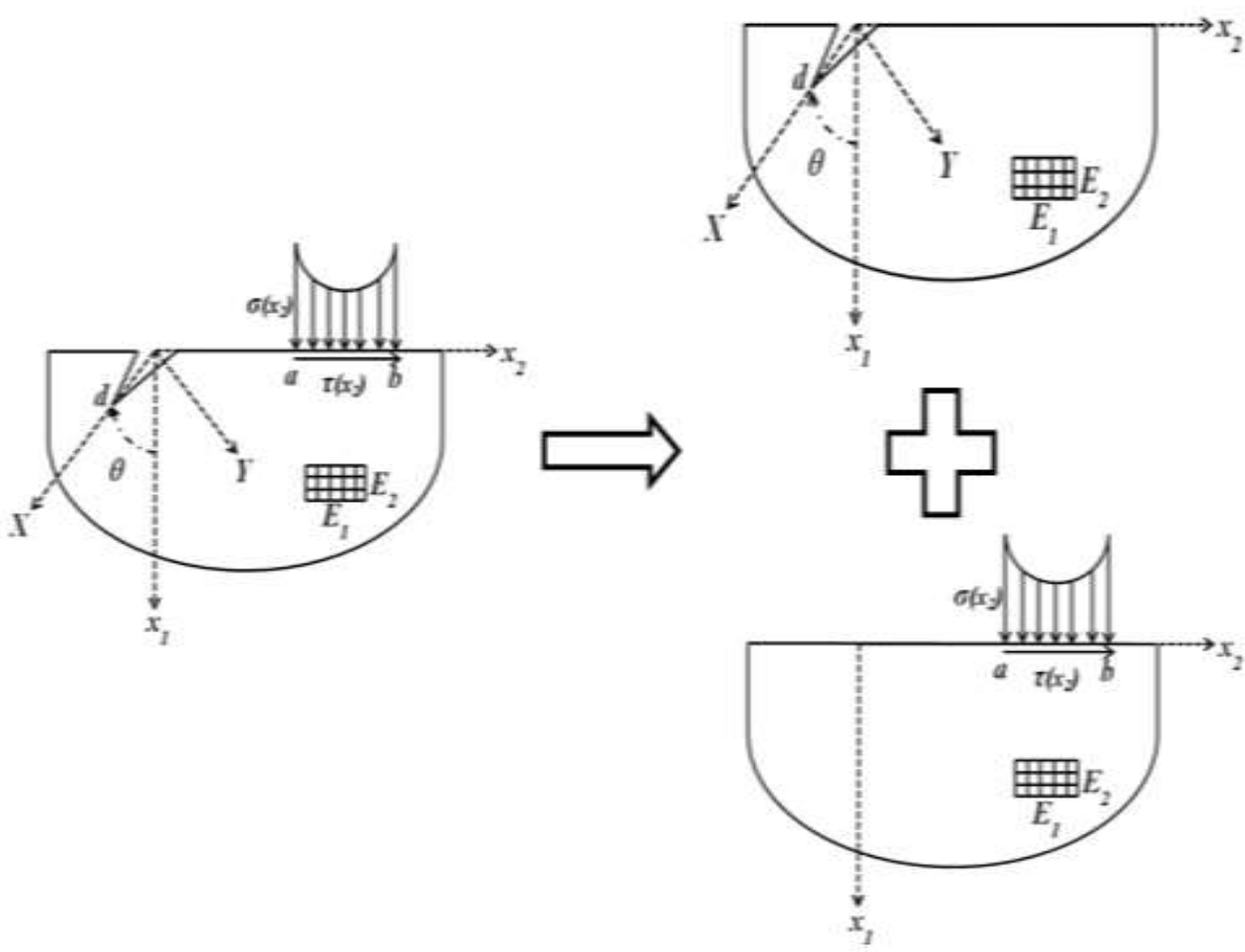

Figure 2.2 Solution Procedure of the Coupled Problem 

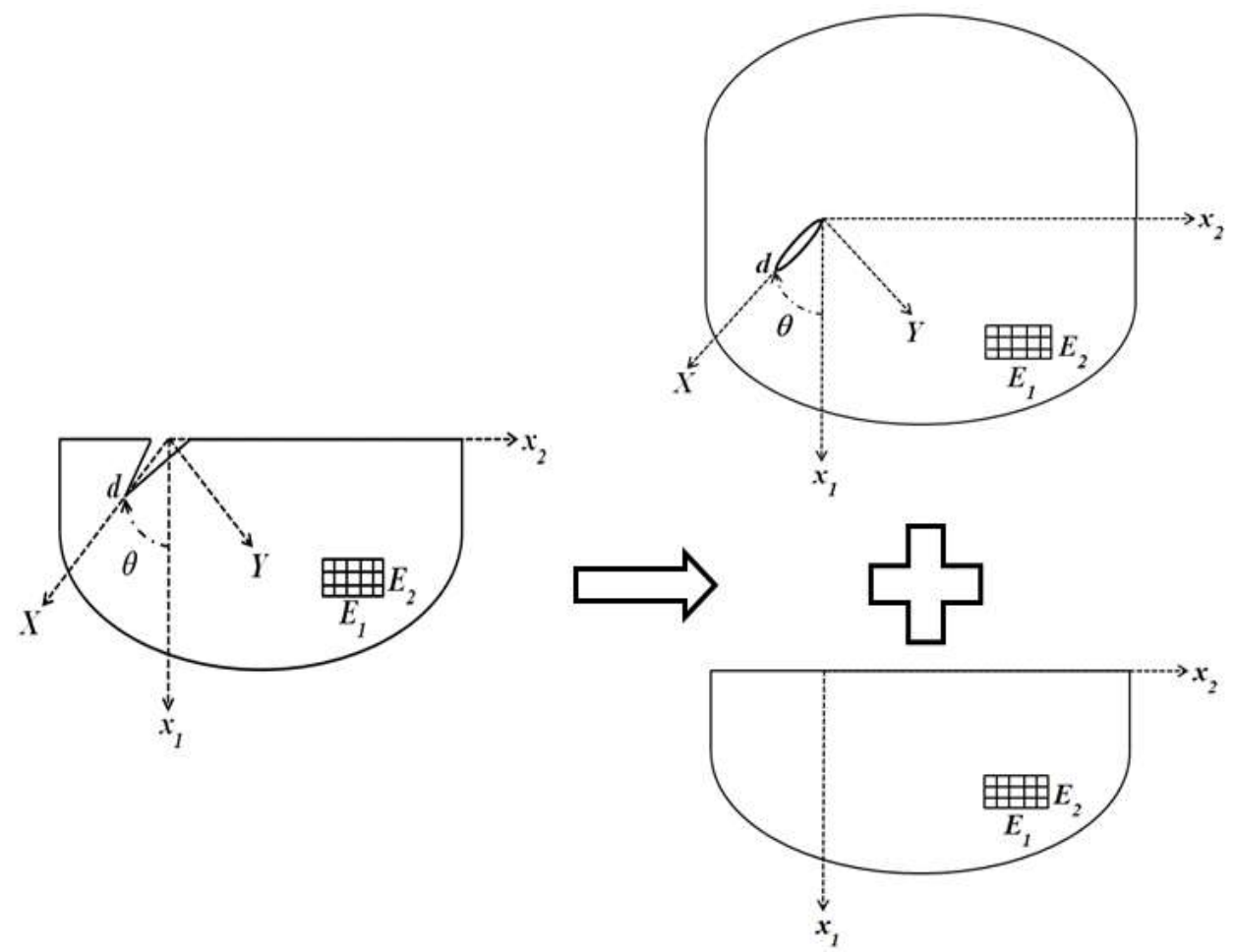

Figure 2.3 Solution Procedure of the Crack Problem

\subsection{The Contact Loading Problem}

The necessary equations for the formulation of the contact loading problem are derived in the dissertation by Sarikaya [2]. The stress and displacement fields in the problem are found using Fourier Transform for $x_{2}$ variable in $x_{1}-x_{2}$ coordinate system in terms of known stress distributions ( $\sigma\left(x_{2}\right)$ and $\tau\left(x_{2}\right)$ ). In Figure 2.4, the configuration of the contact loading problem is given. The formulation relies on the representation of displacement fields as Fourier Integrals.

$u_{1}\left(x_{1}, x_{2}\right)=\frac{1}{2 \pi} \int_{-\infty}^{\infty} U_{1}\left(x_{1}, \rho\right) \exp \left(i \rho x_{2}\right) d \rho$ 


$$
u_{2}\left(x_{1}, x_{2}\right)=\frac{1}{2 \pi} \int_{-\infty}^{\infty} U_{2}\left(x_{1}, \rho\right) \exp \left(i \rho x_{2}\right) d \rho
$$

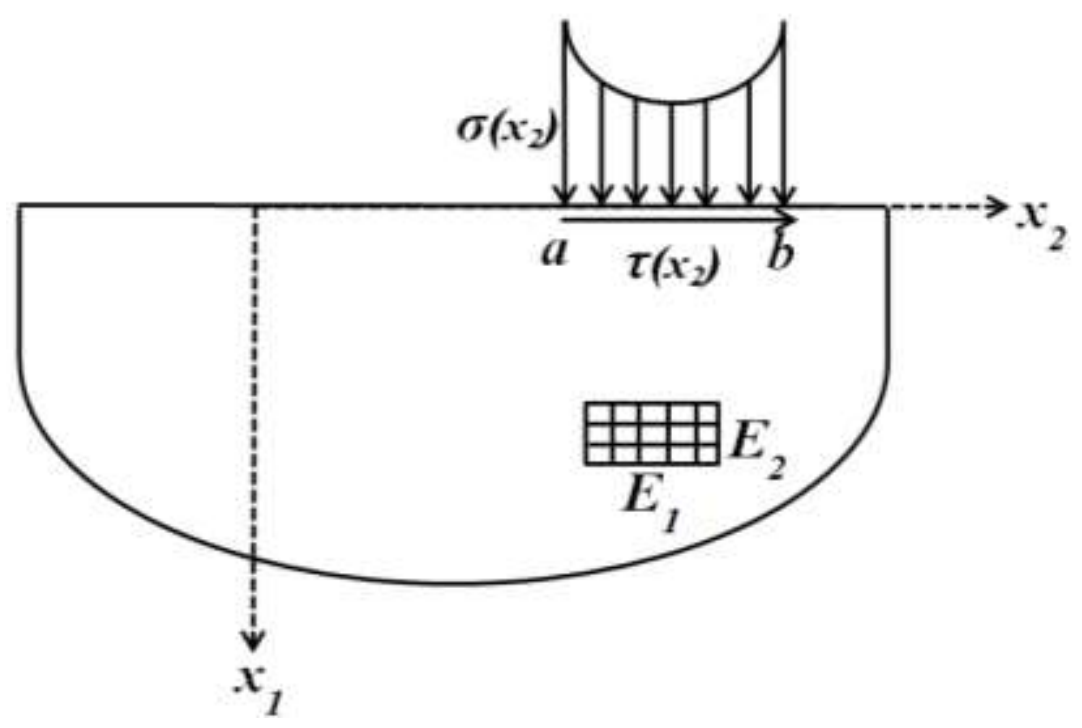

Figure 2.4 Geometry of Contact Loading Problem

This means two partial differential equations in $(2.4 \mathrm{i}-\mathrm{j})$ can be transformed to ordinary differential equations using Fourier Transform for $x_{2}$ variable. Then, the solutions for $U_{1}$ and $U_{2}$ are searched in the form of $\exp \left(s x_{1}\right)$ and the resulting equations are written in the matrix form.

$$
\begin{aligned}
& C_{11} \frac{\partial^{2} U_{1}}{\partial x_{1}{ }^{2}}-\rho^{2} \frac{C_{66}}{2} U_{1}+\left(C_{12}+\frac{C_{66}}{2}\right) i \rho \frac{\partial U_{2}}{\partial x_{1}}=0 \\
& \left(C_{12}+\frac{C_{66}}{2}\right) i \rho \frac{\partial U_{1}}{\partial x_{1}}+\frac{C_{66}}{2} \frac{\partial^{2} U_{2}}{\partial x_{1}{ }^{2}}-\rho^{2} C_{22} U_{2}=0
\end{aligned}
$$


$\left[\begin{array}{ll}\left(C_{11} s^{2}-\frac{C_{66}}{2} \rho^{2}\right) & \left(C_{12}+\frac{C_{66}}{2}\right) i \rho s \\ \left(C_{12}+\frac{C_{66}}{2}\right) i \rho s & \left(\frac{C_{66}}{2} s^{2}-C_{22} \rho^{2}\right)\end{array}\right]\left\{\begin{array}{l}U_{1} \\ U_{2}\end{array}\right\}=0$

$s^{4}+s^{2} s_{1}+s_{2}=0$

$\varsigma_{1}=\frac{2\left(-C_{11} C_{22}+C_{12}^{2}+C_{12} C_{66}\right) \rho^{2}}{C_{11} C_{66}} \quad \varsigma_{2}=\frac{\rho^{4} C_{22}}{C_{11}}$

For $\varsigma_{1}<0, \varsigma_{1}^{2}-4 \varsigma_{2}>0,\left|\varsigma_{1}\right|>\sqrt{\varsigma_{1}^{2}-4 \varsigma_{2}} \quad$ Type 1 Material

A characteristic equation is found by equating the determinant of the matrix in equation (2.7a) to zero. Now, the exact solutions for $U_{1}$ and $U_{2}$ can be obtained by solving the characteristic equation in (2.7b). Delale and Erdogan [3] and Sarikaya [2] classify the orthotropic materials in two groups depending on the roots of this characteristic equation. If the condition related to material parameters given by equation (2.7e) is satisfied, in other words, the characteristic equation of the material gives four real roots; it is classified as type 1 orthotropic material in practical engineering applications. Otherwise, if the roots are complex, the material is called as type 2 .

Orthotropic materials used in tribological applications such as composite structures, coatings and thin films generally possess the characteristics of type 1 material. Hence, Sarikaya [2] worked on type 1 materials. In this study, the main objective is to add the effects of crack orientation angle and crack closure to Sarikaya [2]'s work. For this reason, type 1 materials are focused on. Thus, the formulation from this point on is related to type 1 materials.

In the previous step, the solutions of $U_{1}$ and $U_{2}$ are found. Now, the displacement components are updated and corresponding stresses are defined. 


$$
\begin{aligned}
& u_{1}\left(x_{1}, x_{2}\right)=\frac{1}{2 \pi} \int_{-\infty}^{\infty} \sum_{j=3}^{4} M_{j} \exp \left(s_{j} x_{1}+i \rho x_{2}\right) d \rho \\
& u_{2}\left(x_{1}, x_{2}\right)=\frac{1}{2 \pi} \int_{-\infty}^{\infty} \sum_{j=3}^{4} M_{j} N_{j} \exp \left(s_{j} x_{1}+i \rho x_{2}\right) d \rho \\
& \sigma_{x_{1} x_{1}}\left(x_{1}, x_{2}\right)=\frac{1}{2 \pi} \int_{-\infty}^{\infty} \sum_{j=3}^{4}\left(C_{11} s_{j}+C_{12} i \rho N_{j}\right) M_{j} \exp \left(s_{j} x_{1}+i \rho x_{2}\right) d \rho \\
& \sigma_{x_{1} x_{2}}\left(x_{1}, x_{2}\right)=\frac{1}{2 \pi} \int_{-\infty}^{\infty} \sum_{j=3}^{4} \frac{C_{66}}{2}\left(s_{j} N_{j}+i \rho\right) M_{j} \exp \left(s_{j} x_{1}+i \rho x_{2}\right) d \rho \\
& \sigma_{x_{2} x_{2}}\left(x_{1}, x_{2}\right)=\frac{1}{2 \pi} \int_{-\infty}^{\infty} \sum_{j=3}^{4}\left(C_{12} s_{j}+C_{22} i \rho N_{j}\right) M_{j} \exp \left(s_{j} x_{1}+i \rho x_{2}\right) d \rho
\end{aligned}
$$

Keep in mind that terms related ( $j=1,2)$ vanish in stress and displacement fields given by equations (2.8a-e) due to the regularity conditions in equations (2.1h-k). The unknown functions of the contact loading problem $N_{j}$ 's and $M_{j}$ 's are obtained through equilibrium equations in (2.7a) and the boundary conditions in (2.1a-e), respectively.

$$
\begin{aligned}
& N_{j}(\rho)=\frac{-C_{11} s_{j}^{2}+\frac{C_{66}}{2} \rho^{2}}{\left(C_{12}+\frac{C_{66}}{2}\right) i \rho s_{j}} \\
& {\left[\begin{array}{l}
M_{3} \\
M_{4}
\end{array}\right]=\left[\begin{array}{ll}
C_{11} s_{3}+C_{12} i \rho N_{3} & C_{11} s_{4}+C_{12} i \rho N_{4} \\
\frac{C_{66}}{2}\left(s_{3} N_{3}+i \rho\right) & \frac{C_{66}}{2}\left(s_{4} N_{4}+i \rho\right)
\end{array}\right]^{-1}\left[\begin{array}{l}
\zeta_{1} \\
\zeta_{2}
\end{array}\right]} \\
& \sum_{j=3}^{4}\left(C_{11} s_{j}+C_{12} i \rho N_{j}\right) M_{j}=\int_{a}^{b} \sigma(t) \exp (-i \rho t) d t \\
& \sum_{j=3}^{4} \frac{C_{66}}{2}\left(s_{j} N_{j}+i \rho\right) M_{j}=\int_{a}^{b} \sigma(t) \eta_{1} \exp (-i \rho t) d t
\end{aligned}
$$


$\zeta_{1}=\int_{a}^{b} \sigma(t) \exp (-i \rho t) d t$

$\zeta_{2}=\eta_{1} \int_{a}^{b} \sigma(t) \exp (-i \rho t) d t$

where the roots of the characteristic equation $s_{j}$ 's $(j=1,4)$ are noted as below:

$$
\begin{array}{llll}
s_{1}=q_{1}|\rho| & \mathfrak{R}\left(s_{1}\right)>0 & s_{2}=q_{2}|\rho| & \Re\left(s_{2}\right)>0 \\
s_{3}=-q_{1}|\rho| & \mathfrak{R}\left(s_{3}\right)<0 & s_{4}=-q_{2}|\rho| & \Re\left(s_{4}\right)<0 \\
q_{1}=\frac{\sqrt{-2 \varsigma_{1}+2 \sqrt{\varsigma_{1}^{2}-4 \varsigma_{2}}}}{2} & q_{2}=\frac{\sqrt{-2 \varsigma_{1}-2 \sqrt{\varsigma_{1}^{2}-4 \varsigma_{2}}}}{2}
\end{array}
$$

Then, $M_{j}$ and $N_{j}$ are substituted to equations (2.8c-e). The order of integration is changed and infinite integrals are evaluated in MAPLE. Eventually, the stresses and the displacement fields are obtained as the integral forms in terms of $\sigma(t)$. By deriving the equations about normal and shear stresses acting along the crack line, this section is completed. The explicit expressions of these stresses are given in Appendix A.

$$
\begin{array}{ll}
\sigma_{Y Y}^{\text {Contact }}(X, 0)=\int_{a}^{b} h_{13}(X, t) \sigma(t) d t & 0<X<d \\
\sigma_{X Y}^{\text {Contact }}(X, 0)=\int_{a}^{b} h_{23}(X, t) \sigma(t) d t & 0<X<d
\end{array}
$$

\subsection{Determination of Loading Function $\left(\sigma\left(x_{2}\right)\right)$}

Indentation of three rigid stamp profiles is used to represent the contact between two solids in this study. It is assumed that the modulus of elasticity of the stamp 
is very large compared to the modulus of elasticity of the half-plane so that the stamp preserves its shape in the contact region. Flat, triangular and circular stamps are selected in this study. A two-step solution procedure is applied. First, the stress distribution in the contact region is determined ignoring the impacts of the presence of the crack. Then, this stress distribution is utilized in the calculation of mixed mode stress intensity factors. Note that the crack and the contact loading problems are considered as an uncoupled form with this two-step solution case. However, the results are expected to be accurate when there is a wide distance between the crack and the loading area [29].

Sarikaya [2] showed that the strengths of singularity at the end points of the contact region $\left(\psi_{1}\right.$ and $\left.\psi_{2}\right)$ depend on the coefficient of friction, the parameters of type 1 orthotropic material and stamp profile.

$$
\begin{aligned}
& \sigma\left(x_{2}\right)=\left(x_{2}-a\right)^{\psi_{1}}\left(b-x_{2}\right)^{\psi_{2}} F\left(x_{2}\right) \\
& e_{10}=-\frac{4 \eta_{1}\left(q_{1} C_{11} q_{2}+q_{1} C_{11} q_{2} d_{12}-q_{1} C_{12} q_{2} d_{11}-C_{12}\right)\left(1+d_{12}\right)}{C_{66} e_{30}} \\
& e_{20}=\frac{2\left(q_{1}+q_{2}\right) d_{11}\left(1+d_{12}\right) q_{1} q_{2}}{e_{30}} \\
& e_{30}=q_{1}^{2} C_{11} q_{2}^{2} d_{11} d_{12}-q_{1}^{2} C_{12} d_{11}^{2} q_{2}^{2}+q_{1}^{2} C_{11} q_{2}^{2} d_{11}+C_{12} q_{1}^{2} d_{11} \\
& \quad-q_{1} C_{11} q_{2} d_{12}-q_{1} C_{11} q_{2} d_{12}^{2}+q_{1} C_{12} q_{2} d_{11} d_{12} \\
& \quad+q_{1} C_{12} q_{2} d_{11}+C_{12} d_{12}+C_{12} q_{2}^{2} d_{11}
\end{aligned}
$$

Note that $d_{11}, d_{12}$ and $d_{22}$ in equations (2.11b-d) are defined as follows:

$$
d_{11}=\frac{2 C_{11}}{C_{66}} \quad d_{12}=\frac{2 C_{12}}{C_{66}} \quad d_{22}=\frac{2 C_{22}}{C_{66}}
$$



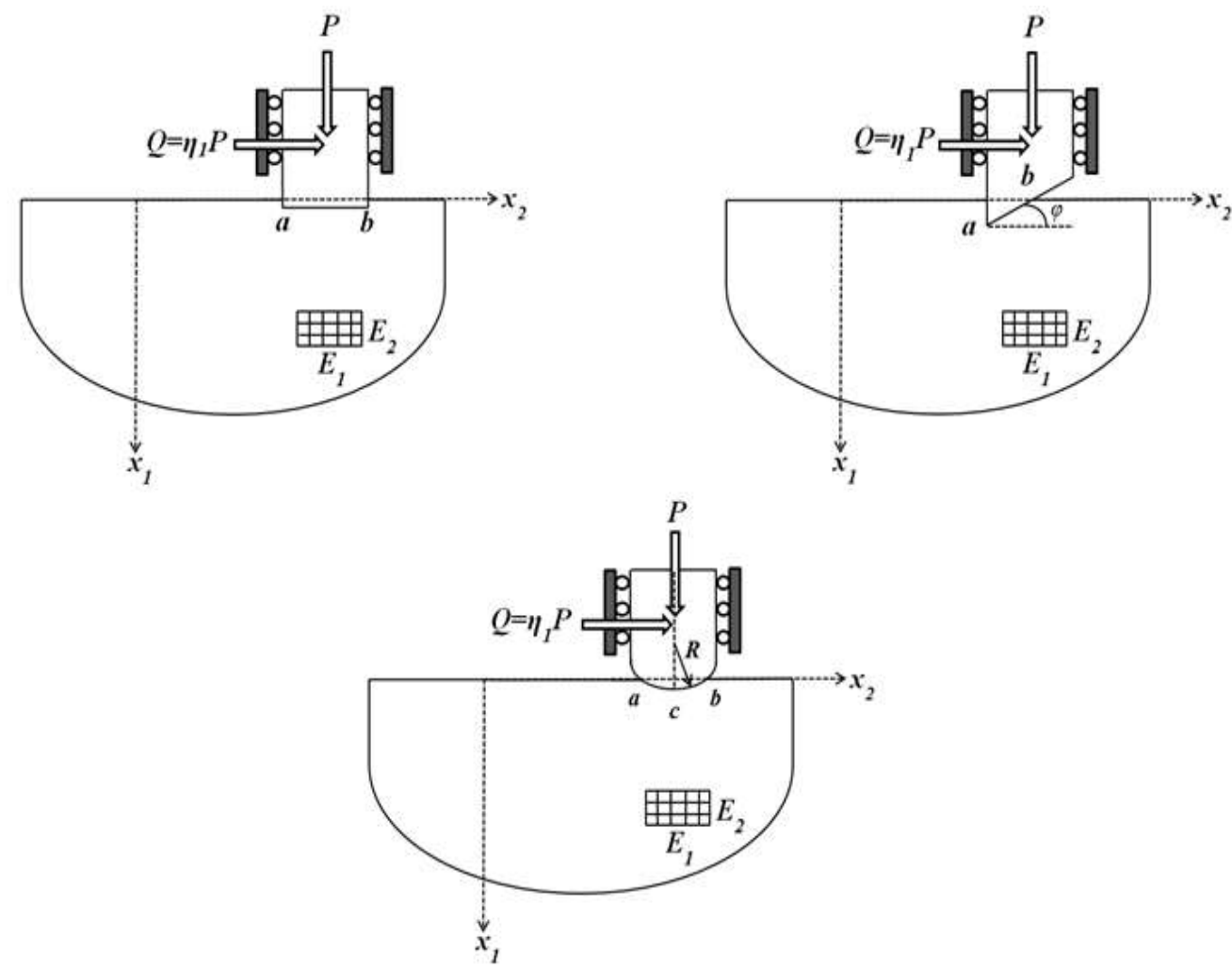

Figure 2.5 Types of Stamp Profiles

Two distinct solutions for both $\psi_{1}$ and $\psi_{2}$ are obtained for the equations in (2.11e-f) between -1 and 1 . The correct values for $\psi_{1}$ and $\psi_{2}$ are chosen by bearing the physics of the problem in mind. If there is a smooth contact at the end point, a positive value should be selected for the strength of singularity. Otherwise, the strength of singularity at that point is negative in the case of sharp contact as the contact stress approaches to infinity. For each stamp profiles, the unknown stress distribution is obtained by expanding it with Jacobi polynomials.

\subsubsection{Pressure Distribution for Flat Stamp}

The solution of the pressure distribution is based on the displacement derivative in the contact region. To begin with, it is observed that there exists a constant 
displacement in the half-plane. Therefore, the displacement derivative equals to zero.

$$
\begin{array}{ll}
u_{1}\left(x_{1}=0, x_{2}\right)=\delta & a<x_{2}<b \\
\frac{\partial u_{1}\left(x_{1}=0, x_{2}\right)}{\partial x_{2}}=0 & a<x_{2}<b
\end{array}
$$

Then, the total force $(P)$, applied by the stamp is defined.

$$
\int_{a}^{b} \sigma\left(x_{2}\right) d x_{2}=-P
$$

The displacement derivative for this problem has already been shown by Sarikaya $[2]$.

$$
\frac{e_{20}}{2} \frac{1}{\pi} \int_{a}^{b} \frac{\sigma(t)}{x_{2}-t} d t+\frac{e_{10}}{2} \sigma\left(x_{2}\right)=0, \quad a<x_{2}<b
$$

At this point, $x_{2}$ and $t$ variables are normalized to $s$ and $r$ variables in order to make the integration limits -1 and 1 . Also, the normalized form of the contact stress distribution $\Phi(s)$ is introduced.

$$
\begin{aligned}
& x_{2}=\frac{(b-a)}{2} s+\frac{(b+a)}{2} \\
& t=\frac{(b-a)}{2} r+\frac{(b+a)}{2} \\
& \Phi(s)=\frac{\sigma\left(\frac{(b-a)}{2} s+\frac{(b+a)}{2}\right)}{P /(b-a)}
\end{aligned}
$$

Equations (2.11a), (2.13c) and (2.13d) are rearranged as follows: 


$$
\begin{array}{ll}
\sigma(r)=(1-r)^{\psi_{2}}(1+r)^{\psi_{1}} \sum_{n=0}^{\infty} A_{n} P_{n}^{\left(\psi_{2}, \psi_{1}\right)}(r) & \begin{array}{c}
\text { where } P_{n}^{\left(\psi_{2}, \psi_{1}\right)}(r) \\
\text { represents Jacobi } \\
\text { Polynomials }
\end{array} \\
\int_{-1}^{1} \Phi(s) d s=-2 & -1<s<1 \\
\frac{e_{20}}{2} \frac{1}{\pi} \int_{-1}^{1} \frac{\sigma(r)}{s-r} d r+\frac{e_{10}}{2} \sigma(s)=0, &
\end{array}
$$

Polynomials

Using the orthogonality properties of Jacobi Polynomials, orthogonality relations in Erdogan et al [36], and the expression in Appendix B, the pressure distribution can be obtained.

$$
\begin{gathered}
\int_{-1}^{1}(1-r)^{\psi_{2}}(1+r)^{\psi_{1}} P_{k}^{\left(\psi_{2}, \psi_{1}\right)}(r) P_{n}^{\left(\psi_{2}, \psi_{1}\right)}(r) d r=\left\{\begin{array}{cl}
\theta_{k}, & n=k \\
0, & n \neq k
\end{array}\right. \\
\theta_{k}= \begin{cases}\frac{2^{\psi_{2}+\psi_{1}+1} \Gamma\left(\psi_{2}+1\right) \Gamma\left(\psi_{1}+1\right)}{\Gamma\left(\psi_{2}+\psi_{1}+2\right)} \text { if } k=0 \text { and } \psi_{2}+\psi_{1}=-1 \\
\frac{2^{\psi_{2}+\psi_{1}+1} \Gamma\left(k+\psi_{2}+1\right) \Gamma\left(k+\psi_{1}+1\right)}{\left(2 k+\psi_{2}+\psi_{1}+1\right) k ! \Gamma\left(k+\psi_{2}+\psi_{1}+1\right)} & \text { Otherwise }\end{cases}
\end{gathered}
$$

Equation (2.14c) is updated as follows:

$$
\sum_{n=1}^{\infty} \frac{e_{20}}{4 \sin \left(\pi \psi_{2}\right)} A_{n} P_{n-1}^{\left(-\psi_{2},-\psi_{1}\right)}(s)=0 \quad A_{n}=0, \text { for } n=1,2,3, \ldots
$$

It can be seen from equation (2.16) that all unknown coefficients of expansion except for $A_{0}$ are zero. Therefore, the only remaining coefficient of expansion that appears in the contact pressure distribution is $A_{0}$ and it is found using the relation in $(2.15 b)$. Eventually, the normalized contact pressure distribution is obtained. 


$$
\begin{array}{ll}
A_{0}=-\frac{2}{\Gamma\left(\psi_{2}+1\right) \Gamma\left(\psi_{1}+1\right)} & \psi_{2}+\psi_{1}=-1 \\
\Phi(s)=-\frac{2(1-s)^{\psi_{2}}(1+s)^{\psi_{1}}}{\Gamma\left(\psi_{2}+1\right) \Gamma\left(\psi_{1}+1\right)} & -1<\psi_{2}<0 \\
& -1<\psi_{1}<0
\end{array}
$$

\subsubsection{Pressure Distribution for Triangular Stamp}

The triangular stamp has a constant displacement derivative throughout the loading region.

$$
\frac{\partial u_{1}\left(x_{1}=0, x_{2}\right)}{\partial x_{2}}=-m \quad m \text { is the slope of the stamp }
$$

The governing equation then becomes as follows:

$$
\frac{e_{20}}{2} \frac{1}{\pi} \int_{a}^{b} \frac{\sigma(t)}{x_{2}-t} d t+\frac{e_{10}}{2} \sigma\left(x_{2}\right)=-m, \quad a<x_{2}<b
$$

Here, the similar procedures are applied to the governing equation in (2.19) and it is observed that the unknown coefficients of expansions excluding $A_{0}$ are zero.

$$
\sum_{n=0}^{\infty} \frac{e_{20}}{2 \sin \left(\pi \psi_{2}\right)} A_{n} P_{n}^{\left(-\psi_{2},-\psi_{1}\right)}(s)=-m \quad A_{n}=0, \text { for } n=1,2,3, \ldots
$$

Note that $A_{0}$ can be defined in terms of $m$ by using equation (2.20). However, it is not desired to have the stamp parameters in the normalized contact stress distribution. Instead of this, it is preferred to apply equation (2.15b) to find $A_{0}$.

$$
\int_{-1}^{1} \Phi(s) d s=-2
$$




$$
\begin{array}{lc}
A_{0}=-\frac{1}{\Gamma\left(\psi_{2}+1\right) \Gamma\left(\psi_{1}+1\right)} & \psi_{2}+\psi_{1}=0 \\
\Phi(s)=-\frac{(1-s)^{\psi_{2}}(1+s)^{\psi_{1}}}{\Gamma\left(\psi_{2}+1\right) \Gamma\left(\psi_{1}+1\right)} & 0<\psi_{2}<1 \\
& -1<\psi_{1}<0
\end{array}
$$

\subsubsection{Pressure Distribution for Circular Stamp}

The displacement derivative for the circular stamp problem is rather complex than other two cases. The proof of the displacement derivative equation is presented in details by Ozatag [37].

$$
\frac{\partial u_{1}\left(x_{1}=0, x_{2}\right)}{\partial x_{2}}=\frac{c-x_{2}}{R}, \quad a<x_{2}<b
$$

In equation (2.22), $c$ and $R$ are the centerline and radius of the stamp, respectively. Again, it is found that the coefficients of expansion starting from $A_{1}$ vanish in the governing equation (2.23).

$$
\begin{aligned}
\sum_{n=0}^{\infty} \frac{e_{20}}{\sin \left(\pi \psi_{2}\right)} & A_{n} P_{n+1}^{\left(-\psi_{2},-\psi_{1}\right)}(s) \\
& =\frac{-(b-a) P_{1}^{\left(-\psi_{2},-\psi_{1}\right)}(s)}{2 R}
\end{aligned}
$$

Once again, it is applied to the orthogonality relation of Jacobi Polynomials in order to eliminate the stamp parameters $(c$ and $R)$ in the normalized contact stress distribution.

$$
\int_{-1}^{1} \Phi(s) d s=-2
$$




$$
\begin{array}{ll}
A_{0}=-\frac{1}{\Gamma\left(\psi_{2}+1\right) \Gamma\left(\psi_{1}+1\right)} & \psi_{2}+\psi_{1}=1 \\
\Phi(s)=-\frac{(1-s)^{\psi_{2}}(1+s)^{\psi_{1}}}{\Gamma\left(\psi_{2}+1\right) \Gamma\left(\psi_{1}+1\right)} & 0<\psi_{2}<1 \\
& 0<\psi_{1}<1
\end{array}
$$

At this point, the loading functions for three different stamp profiles are determined. Sample contact stress distributions are illustrated in Figure 2.6.

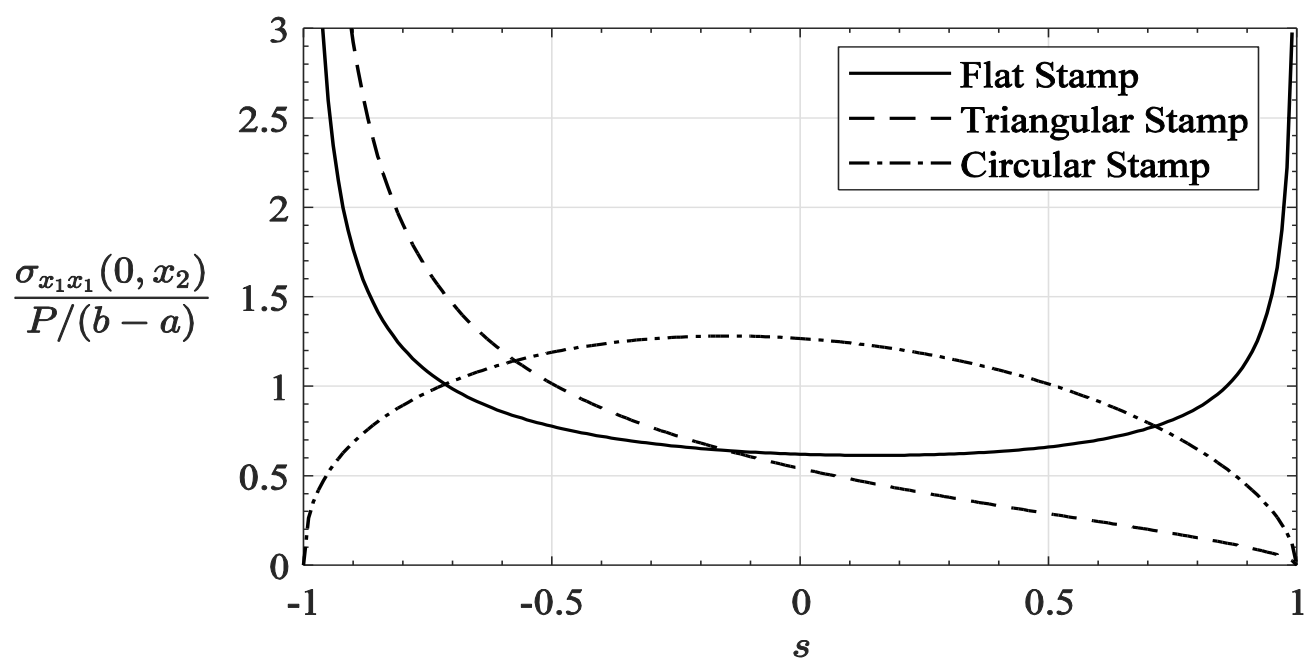

Figure 2.6 Sample Pressure Distributions for Plane Stress Case, $\eta_{1}=0.6$

\subsection{The Crack Problem}

As shown in Figure 2.7, the crack faces are exposed to surface stresses, originating from the stresses found from contact loading problem. Fully open crack assumption states that crack faces are stress free. Thus, the main boundary conditions of the crack problem read: 


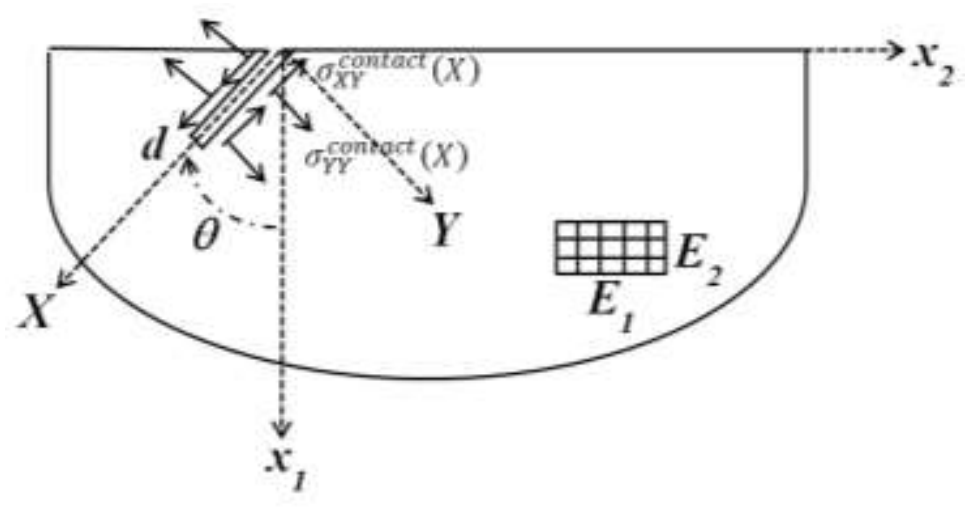

Figure 2.7 Geometry of Crack Problem

$$
\begin{array}{ll}
\sigma_{Y Y}(X, 0)=\sigma_{Y Y}^{\text {crack }}(X, 0)+\int_{a}^{b} h_{13}(X, t) \sigma(t) d t=0, & 0<X<d \\
\sigma_{X Y}(X, 0)=\sigma_{X Y}^{c r a c k}(X, 0)+\int_{a}^{b} h_{23}(X, t) \sigma(t) d t=0, & 0<X<d \\
\sigma_{x_{1} x_{1}}\left(0, x_{2}\right)=\sigma_{x_{1} x_{2}}\left(0, x_{2}\right)=0, & -\infty<x_{2}<\infty \\
\frac{Q_{66}}{2} \frac{\partial}{\partial X}\left(V^{(+i)}\left(X, 0^{+}\right)-V^{(-i)}\left(X, 0^{-}\right)\right)=f_{1}(X) & 0<X<d \\
\frac{Q_{66}}{2} \frac{\partial}{\partial X}\left(U^{(+i)}\left(X, 0^{+}\right)-U^{(-i)}\left(X, 0^{-}\right)\right)=f_{2}(X) & 0<X<d
\end{array}
$$

where $\sigma_{Y Y}^{\text {crack }}(X, 0)$ and $\sigma_{X Y}^{\text {crack }}(X, 0)$ in equations $(2.25 \mathrm{a})$ and $(2.25 \mathrm{~b})$ are the stresses written in terms of unknown derivatives of crack surface displacement functions $\left(f_{1}(X)\right.$ and $\left.f_{2}(X)\right)$, stemmed from the solution of crack problem. In this section, the infinite plane-half plane superposition method in Figure 2.3 can be applied to crack problem. Infinite plane problem and half plane problem are depicted in Figure 2.8 and Figure 2.9, respectively. Infinite plane problem is considered in $X-Y$ coordinate system. Therefore, coordinate transformation is 
applied to obtain the stiffness matrix of the material in $X-Y$ coordinate system. Fourier Transforms of the equilibrium equations can be taken in $X$-direction. By solving the resulting system of ordinary differential equations, the stress and displacement expressions for both half-planes $Y>0$ and $Y<0$ can be obtained. The superscripts $(i)$ and $(h)$ in displacements and stresses in this section designate the solutions for infinite plane and half plane problems, respectively.

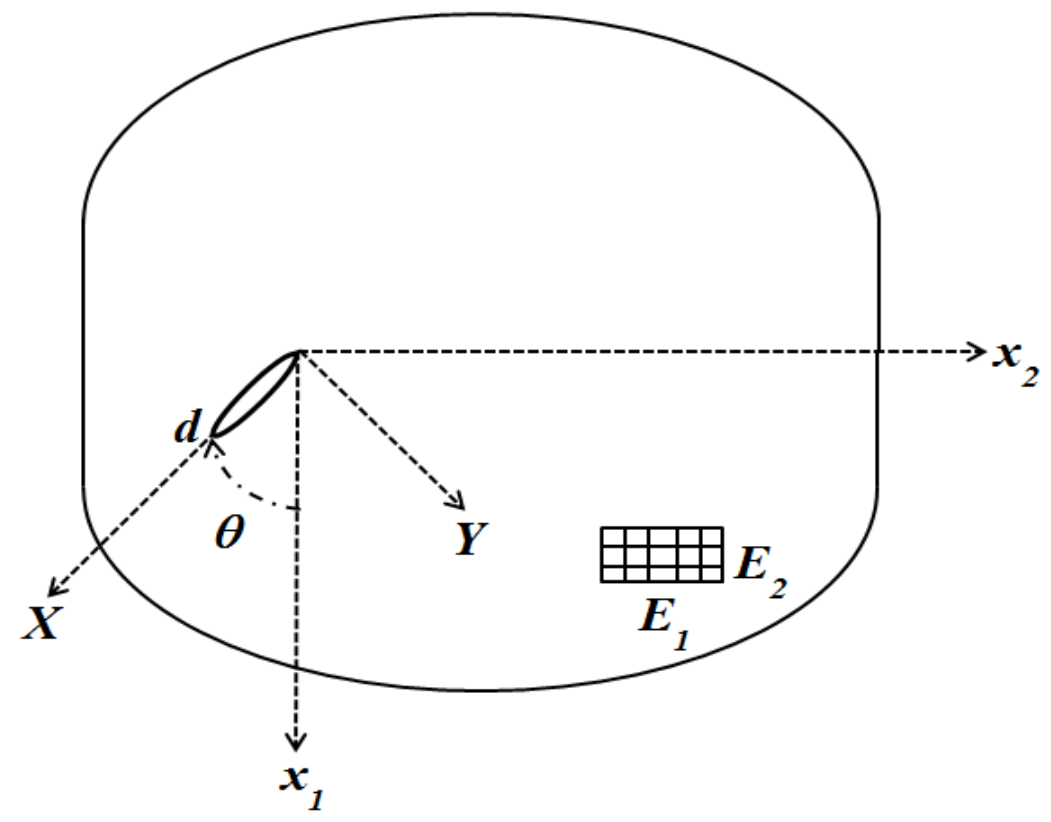

Figure 2.8 Infinite Plane Problem with a Crack

At this point, a simple stress transformation procedure is given for orthotropic materials.

$R=\left[\begin{array}{ccc}C_{11} & C_{12} & 0 \\ C_{12} & C_{22} & 0 \\ 0 & 0 & C_{66}\end{array}\right]$ 


$$
\begin{aligned}
& {\left[\begin{array}{l}
\sigma_{X X} \sigma_{X Y} \\
\sigma_{X Y} \sigma_{Y Y}
\end{array}\right]=\left[\begin{array}{cr}
\cos (\theta) & -\sin (\theta) \\
\sin (\theta) & \cos (\theta)
\end{array}\right]\left[\begin{array}{l}
\sigma_{x_{1} x_{1}} \sigma_{x_{1} x_{2}} \\
\sigma_{x_{1} x_{2}} \sigma_{x_{2} x_{2}}
\end{array}\right]\left[\begin{array}{rr}
\cos (\theta) & \sin (\theta) \\
-\sin (\theta) & \cos (\theta)
\end{array}\right]} \\
& {\left[\begin{array}{l}
\sigma_{X X} \\
\sigma_{Y Y} \\
\sigma_{X Y}
\end{array}\right]=\left[\begin{array}{ccr}
\cos ^{2}(\theta) & \sin ^{2}(\theta) & -\sin (2 \theta) \\
\sin ^{2}(\theta) & \cos ^{2}(\theta) & \sin (2 \theta) \\
\cos (\theta) \sin (\theta) & -\cos (\theta) \sin (\theta) & \cos (2 \theta)
\end{array}\right]\left[\begin{array}{l}
\sigma_{x_{1} x_{1}} \\
\sigma_{x_{2} x_{2}} \\
\sigma_{x_{1} x_{2}}
\end{array}\right]} \\
& T=\left[\begin{array}{ccr}
\cos ^{2}(\theta) & \sin ^{2}(\theta) & -\sin (2 \theta) \\
\sin ^{2}(\theta) & \cos ^{2}(\theta) & \sin (2 \theta) \\
\cos (\theta) \sin (\theta) & -\cos (\theta) \sin (\theta) & \cos (2 \theta)
\end{array}\right] \\
& {\left[\begin{array}{l}
\sigma_{X X} \\
\sigma_{Y Y} \\
\sigma_{X Y}
\end{array}\right]=T * R * T^{-1}\left[\begin{array}{l}
\varepsilon_{X X} \\
\varepsilon_{Y Y} \\
\varepsilon_{X Y}
\end{array}\right]} \\
& Q=T * R * T^{-1}
\end{aligned}
$$

After the transformation, the constitutive relations take the following form.

$$
\left[\begin{array}{l}
\sigma_{X X} \\
\sigma_{Y Y} \\
\sigma_{X Y}
\end{array}\right]=\left[\begin{array}{lll}
Q_{11} & Q_{12} & Q_{16} \\
Q_{21} & Q_{22} & Q_{26} \\
Q_{61} & Q_{62} & Q_{66}
\end{array}\right]\left[\begin{array}{c}
\varepsilon_{X X} \\
\varepsilon_{Y Y} \\
\varepsilon_{X Y}
\end{array}\right]
$$

The displacement components for infinite plane problem are in the form of Fourier Integrals.

$$
\begin{aligned}
& U^{(i)}(X, Y)=\frac{1}{2 \pi} \int_{-\infty}^{\infty} U_{1}^{(i)}(\omega, Y) \exp (i \omega X) d \omega \\
& V^{(i)}(X, Y)=\frac{1}{2 \pi} \int_{-\infty}^{\infty} V_{1}^{(i)}(\omega, Y) \exp (i \omega X) d \omega
\end{aligned}
$$

The equilibrium equations are given as follows:

$$
\begin{gathered}
Q_{11} \frac{\partial^{2} U^{(i)}}{\partial X^{2}}+\frac{Q_{66}}{2} \frac{\partial^{2} U^{(i)}}{\partial Y^{2}}+\left(\frac{Q_{16}}{2}+Q_{61}\right) \frac{\partial^{2} U^{(i)}}{\partial X \partial Y}+Q_{62} \frac{\partial^{2} V^{(i)}}{\partial Y^{2}} \\
+\frac{Q_{16}}{2} \frac{\partial^{2} V^{(i)}}{\partial X^{2}}+\left(\frac{Q_{66}}{2}+Q_{12}\right) \frac{\partial^{2} V^{(i)}}{\partial X \partial Y}=0
\end{gathered}
$$




$$
\begin{gathered}
Q_{61} \frac{\partial^{2} U^{(i)}}{\partial X^{2}}+\frac{Q_{26}}{2} \frac{\partial^{2} U^{(i)}}{\partial Y^{2}}+\left(\frac{Q_{66}}{2}+Q_{21}\right) \frac{\partial^{2} U^{(i)}}{\partial X \partial Y}+Q_{22} \frac{\partial^{2} V^{(i)}}{\partial Y^{2}} \\
+\frac{Q_{66}}{2} \frac{\partial^{2} V^{(i)}}{\partial X^{2}}+\left(\frac{Q_{26}}{2}+Q_{62}\right) \frac{\partial^{2} V^{(i)}}{\partial X \partial Y}=0
\end{gathered}
$$

Searching a solution for $U_{1}{ }^{(i)}$ and $V_{1}^{(i)}$ in the form of $\exp (n Y)$ and using the Fourier Transform, the equilibrium equations can be updated as follows:

$$
\begin{aligned}
& \left(-\omega^{2} Q_{11}+\frac{Q_{66}}{2} n^{2}+\left(\frac{Q_{16}}{2}+Q_{61}\right) i \omega n\right) U_{1}^{(i)} \\
& +\left(Q_{62} n^{2}-\frac{Q_{16}}{2} \omega^{2}+\left(\frac{Q_{66}}{2}+Q_{12}\right) i \omega n\right) V_{1}^{(i)}=0 \\
& \left(-\omega^{2} Q_{61}+\frac{Q_{26}}{2} n^{2}+\left(\frac{Q_{66}}{2}+Q_{21}\right) i \omega n\right) U_{1}^{(i)} \\
& +\left(Q_{22} n^{2}-\frac{Q_{66}}{2} \omega^{2}+\left(\frac{Q_{26}}{2}+Q_{62}\right) i \omega n\right) V_{1}^{(i)}=0
\end{aligned}
$$

Equations (2.28a) and (2.28b) can be written in the matrix form.

$$
\begin{aligned}
& {\left[\begin{array}{ll}
L_{1} & L_{2} \\
L_{3} & L_{4}
\end{array}\right]\left\{\begin{array}{l}
U_{1}^{(i)} \\
V_{1}^{(i)}
\end{array}\right\}=0} \\
& L_{1}=-\omega^{2} Q_{11}+\frac{Q_{66}}{2} n^{2}+\left(\frac{Q_{16}}{2}+Q_{61}\right) i \omega n \\
& L_{2}=Q_{62} n^{2}-\frac{Q_{16}}{2} \omega^{2}+\left(\frac{Q_{66}}{2}+Q_{12}\right) i \omega n \\
& L_{3}=-\omega^{2} Q_{61}+\frac{Q_{26}}{2} n^{2}+\left(\frac{Q_{66}}{2}+Q_{21}\right) i \omega n \\
& L_{4}=Q_{22} n^{2}-\frac{Q_{66}}{2} \omega^{2}+\left(\frac{Q_{26}}{2}+Q_{62}\right) i \omega n
\end{aligned}
$$


The determinant of the matrix in equation $(2.28 \mathrm{c})$ will give the solutions for $U_{1}^{(i)}$ and $V_{1}^{(i)}$.

$$
L_{1} L_{4}-L_{2} L_{3}=0
$$

Once the roots of the characteristic equation in (2.29) are obtained, the relations for both half-planes $Y>0$ and $Y<0$ are noted as follows:

For the half-plane $Y<0$

$$
\begin{aligned}
& U^{(-i)}(X, Y)=\frac{1}{2 \pi} \int_{-\infty}^{\infty} \sum_{j=1}^{2} c_{j} \exp \left(n_{j} Y+i \omega X\right) d \omega \\
& V^{(-i)}(X, Y)=\frac{1}{2 \pi} \int_{-\infty}^{\infty} \sum_{j=1}^{2} c_{j} A_{j} \exp \left(n_{j} Y+i \omega X\right) d \omega \\
& \sigma_{X X}^{(-i)}(X, Y)=\frac{1}{2 \pi} \int_{-\infty}^{\infty} \sum_{j=1}^{2} S_{11} c_{j} \exp \left(n_{j} Y+i \omega X\right) d \omega \\
& \sigma_{Y Y}^{(-i)}(X, Y)=\frac{1}{2 \pi} \int_{-\infty}^{\infty} \sum_{j=1}^{2} S_{21} c_{j} \exp \left(n_{j} Y+i \omega X\right) d \omega \\
& \sigma_{X Y}^{(-i)}(X, Y)=\frac{1}{2 \pi} \int_{-\infty}^{\infty} \sum_{j=1}^{2} S_{61} c_{j} \exp \left(n_{j} Y+i \omega X\right) d \omega
\end{aligned}
$$

For the half-plane $Y>0$

$$
U^{(+i)}(X, Y)=\frac{1}{2 \pi} \int_{-\infty}^{\infty} \sum_{j=3}^{4} c_{j} \exp \left(n_{j} Y+i \omega X\right) d \omega
$$




$$
\begin{aligned}
& V^{(+i)}(X, Y)=\frac{1}{2 \pi} \int_{-\infty}^{\infty} \sum_{j=3}^{4} c_{j} A_{j} \exp \left(n_{j} Y+i \omega X\right) d \omega \\
& \sigma_{X X}^{(+i)}(X, Y)=\frac{1}{2 \pi} \int_{-\infty}^{\infty} \sum_{j=3}^{4} S_{11} c_{j} \exp \left(n_{j} Y+i \omega X\right) d \omega \\
& \sigma_{Y Y}^{(+i)}(X, Y)=\frac{1}{2 \pi} \int_{-\infty}^{\infty} \sum_{j=3}^{4} S_{21} c_{j} \exp \left(n_{j} Y+i \omega X\right) d \omega \\
& \sigma_{X Y}^{(+i)}(X, Y)=\frac{1}{2 \pi} \int_{-\infty}^{\infty} \sum_{j=3}^{4} S_{61} c_{j} \exp \left(n_{j} Y+i \omega X\right) d \omega \\
& S_{11}=\left(Q_{11} i \omega+Q_{12} A_{j} n_{j}+\frac{Q_{16}}{2}\left(A_{j} i \omega+n_{j}\right)\right) \\
& S_{21}=\left(Q_{21} i \omega+Q_{22} A_{j} n_{j}+\frac{Q_{26}}{2}\left(A_{j} i \omega+n_{j}\right)\right) \\
& S_{61}=\left(Q_{61} i \omega+Q_{62} A_{j} n_{j}+\frac{Q_{66}}{2}\left(A_{j} i \omega+n_{j}\right)\right)
\end{aligned}
$$

where $A_{j}$ 's can be obtained using one of the equilibrium equations and index $j$ is selected based on the roots $n_{j}$ 's. Using the following system of linear equations, $c_{j}$ 's $(j=1 . .4)$ can also be found.

$$
\begin{array}{ll}
A_{j}=\frac{\left(\omega^{2} Q_{11}-\frac{Q_{66}}{2} n_{j}^{2}-\left(\frac{Q_{16}}{2}+Q_{61}\right) i \omega n_{j}\right)}{\left(Q_{62} n_{j}^{2}-\frac{Q_{16}}{2} \omega^{2}+\left(\frac{Q_{66}}{2}+Q_{12}\right) i \omega n_{j}\right)} & \\
\sigma_{Y Y}^{(+i)}\left(X, Y=0^{+}\right)=\sigma_{Y Y}^{(-i)}\left(X, Y=0^{-}\right) & 0<X<\infty \\
\sigma_{X Y}^{(+i)}\left(X, Y=0^{+}\right)=\sigma_{X Y}^{(-i)}\left(X, Y=0^{-}\right) & 0<X<\infty \\
\frac{Q_{66}}{2} \frac{\partial}{\partial X}\left(V^{(+i)}\left(X, 0^{+}\right)-V^{(-i)}\left(X, 0^{-}\right)\right)=f_{1}(X) & 0<X<d \\
\frac{Q_{66}}{2} \frac{\partial}{\partial X}\left(U^{(+i)}\left(X, 0^{+}\right)-U^{(-i)}\left(X, 0^{-}\right)\right)=f_{2}(X) & 0<X<d
\end{array}
$$




$$
\begin{aligned}
& \sum_{j=3}^{4} S_{21} c_{j}(\omega)-\sum_{j=1}^{2} S_{21} c_{j}(\omega)=0 \\
& \sum_{j=3}^{4} S_{61} c_{j}(\omega)-\sum_{j=1}^{2} S_{61} c_{j}(\omega)=0 \\
& \sum_{j=3}^{4} \frac{Q_{66}}{2} i \omega A_{j} c_{j}(\omega)-\sum_{j=1}^{2} \frac{Q_{66}}{2} i \omega A_{j} c_{j}(\omega)=\int_{0}^{d} f_{1}(t) \exp (-i \omega t) d t \\
& \sum_{j=3}^{4} \frac{Q_{66}}{2} i \omega c_{j}(\omega)-\sum_{j=1}^{2} \frac{Q_{66}}{2} i \omega c_{j}(\omega)=\int_{0}^{d} f_{2}(t) \exp (-i \omega t) d t
\end{aligned}
$$

In practical engineering applications of type 1 materials, it is observed that the solution of equation (2.29) gives four complex $n$ values. Two of them are with positive real parts and two of them are with negative real parts.

$$
\begin{array}{ll}
n_{1}=q_{3}|\omega|+q_{4} i \omega & \Re\left(n_{1}\right)>0 \\
n_{2}=q_{5}|\omega|-q_{6} i \omega & \Re\left(n_{2}\right)>0 \\
n_{3}=-q_{3}|\omega|+q_{4} i \omega & \Re\left(n_{3}\right)<0 \\
n_{4}=-q_{5}|\omega|-q_{6} i \omega & \Re\left(n_{4}\right)<0
\end{array}
$$

where $q_{3}, q_{4}, q_{5}$ and $q_{6}$ are positive real numbers and lengthy functions of material parameters and crack angle. Substituting $c_{j}$ 's into related equations, and using MAPLE in the evaluation of infinite integrals, the stress and displacement equations in (2.30a-e) and (2.31a-e) can be evaluated.

Now, stress fields obtained for the infinite plane problem are transformed to $x_{1}$ $x_{2}$ coordinate system. For the half plane problem, the equilibrium equations are solved in $x_{1}-x_{2}$ coordinate system using Fourier Transform of $x_{2}$ variable. Eventually, the stress free material surface conditions in $(2.25 \mathrm{c}-\mathrm{d})$ are satisfied with the summation of stress fields for both infinite plane problem and half plane problem. 


$$
\begin{gathered}
\sigma_{x_{1} x_{1}}^{(h)}\left(0, x_{2}\right)+\left[\begin{array}{lll}
\cos ^{2}(\theta) & \sin ^{2}(\theta) & \sin (2 \theta)
\end{array}\right]\left[\begin{array}{l}
\sigma_{X X}^{(i)}(X, Y) \\
\sigma_{Y Y}^{(i)}(X, Y) \\
\sigma_{X Y}^{(i)}(X, Y)
\end{array}\right]=0 \\
\sigma_{x_{1} x_{2}}^{(h)}\left(0, x_{2}\right)+\left[\begin{array}{lll}
-\sin (2 \theta) / 2 & \sin (2 \theta) / 2 & \cos (2 \theta)
\end{array}\right]\left[\begin{array}{l}
\sigma_{X X}^{(i)}(X, Y) \\
\sigma_{Y Y}^{(i)}(X, Y) \\
\sigma_{X Y}^{(i)}(X, Y)
\end{array}\right]=0
\end{gathered}
$$

The displacement components are in the form of Fourier Integrals.

$$
\begin{aligned}
& U^{(h)}\left(x_{1}, x_{2}\right)=\frac{1}{2 \pi} \int_{-\infty}^{\infty} U_{1}^{(h)}\left(x_{1}, \alpha\right) \exp \left(i \alpha x_{2}\right) d \alpha \\
& V^{(h)}\left(x_{1}, x_{2}\right)=\frac{1}{2 \pi} \int_{-\infty}^{\infty} V_{1}^{(h)}\left(x_{1}, \alpha\right) \exp \left(i \alpha x_{2}\right) d \alpha
\end{aligned}
$$

The equilibrium equations in $x_{1}-x_{2}$ coordinate system can be written as follows:

$$
\begin{aligned}
& C_{11} \frac{\partial^{2} U^{(h)}}{\partial x_{1}{ }^{2}}+\frac{C_{66}}{2} \frac{\partial^{2} U^{(h)}}{\partial x_{2}{ }^{2}}+\left(C_{12}+\frac{C_{66}}{2}\right) \frac{\partial^{2} V^{(h)}}{\partial x_{1} \partial x_{2}}=0 \\
& \left(C_{12}+\frac{C_{66}}{2}\right) \frac{\partial^{2} U^{(h)}}{\partial x_{1} \partial x_{2}}+\frac{C_{66}}{2} \frac{\partial^{2} V^{(h)}}{\partial x_{1}{ }^{2}}+C_{22} \frac{\partial^{2} V^{(h)}}{\partial x_{2}{ }^{2}}=0
\end{aligned}
$$

Searching a solution for $U_{1}^{(h)}$ and $V_{1}^{(h)}$ in the form of $\exp \left(p x_{1}\right)$ and using the Fourier Transform, the equilibrium equations can be updated as follows:

$$
\begin{aligned}
& \left(C_{11} p^{2}-\frac{C_{66}}{2} \alpha^{2}\right) U_{1}{ }^{(h)}+\left(C_{12}+\frac{C_{66}}{2}\right) i \alpha p V_{1}^{(h)}=0 \\
& \left(C_{12}+\frac{C_{66}}{2}\right) i \alpha p U_{1}{ }^{(h)}+\left(\frac{C_{66}}{2} p^{2}-C_{22} \alpha^{2}\right) V_{1}^{(h)}=0
\end{aligned}
$$


Equations (2.38a) and (2.38b) can be rearranged in the matrix form.

$$
\begin{array}{ll}
{\left[\begin{array}{ll}
K_{1} & K_{2} \\
K_{3} & K_{4}
\end{array}\right]\left\{\begin{array}{c}
U_{1}^{(h)} \\
V_{1}^{(h)}
\end{array}\right\}=0} & K_{2}=\left(C_{12}+\frac{C_{66}}{2}\right) i \alpha p \\
K_{1}=\left(C_{11} p^{2}-\frac{C_{66}}{2} \alpha^{2}\right) & K_{4}=\left(\frac{C_{66}}{2} p^{2}-C_{22} \alpha^{2}\right) \\
K_{3}=\left(C_{12}+\frac{C_{66}}{2}\right) i \alpha p &
\end{array}
$$

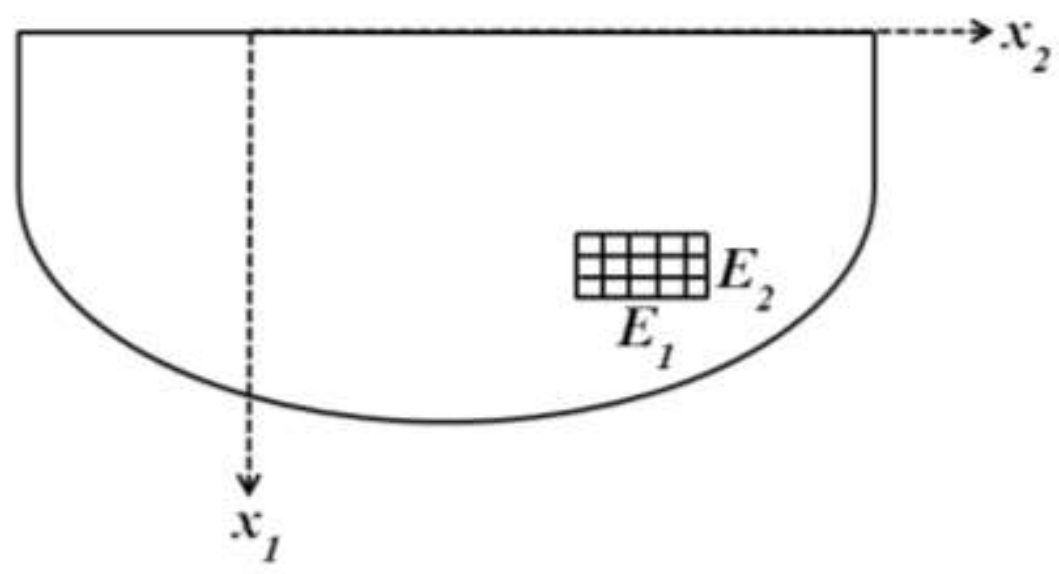

Figure 2.9 Half Plane Problem

The determinant of matrix in $(2.38 \mathrm{c})$ will give the solution for $U_{1}^{(h)}$ and $V_{1}^{(h)}$.

$$
K_{1} K_{4}-K_{2} K_{3}=0
$$

Once the roots of the characteristic equation in (2.39) are obtained, all field quantities are noted as follows: 
For the half-plane $x_{1}>0$

$$
\begin{aligned}
& U^{(h)}\left(x_{1}, x_{2}\right)=\frac{1}{2 \pi} \int_{\infty}^{\infty} \sum_{j=3}^{4} b_{j} \exp \left(p_{j} x_{1}+i \alpha x_{2}\right) d \alpha \\
& V^{(h)}\left(x_{1}, x_{2}\right)=\frac{1}{2 \pi} \int_{-\infty}^{\infty} \sum_{j=3}^{4} b_{j} D_{j} \exp \left(p_{j} x_{1}+i \alpha x_{2}\right) d \alpha \\
& \sigma_{x_{1} x_{1}}^{(h)}\left(x_{1}, x_{2}\right)=\frac{1}{2 \pi} \int_{-\infty}^{\infty} \sum_{j=3}^{4}\left(C_{11} p_{j}+C_{12} i \alpha D_{j}\right) b_{j} \exp \left(p_{j} x_{1}+i \alpha x_{2}\right) d \alpha \\
& \sigma_{x_{2} x_{2}}^{(h)}\left(x_{1}, x_{2}\right)=\frac{1}{2 \pi} \int_{-\infty}^{\infty} \sum_{j=3}^{4}\left(C_{12} p_{j}+C_{22} i \alpha D_{j}\right) b_{j} \exp \left(p_{j} x_{1}+i \alpha x_{2}\right) d \alpha \\
& \sigma_{x_{1} x_{2}}^{(h)}\left(x_{1}, x_{2}\right)=\frac{1}{2 \pi} \int_{-\infty}^{\infty} \sum_{j=3}^{4} \frac{C_{66}}{2}\left(i \alpha+D_{j} p_{j}\right) b_{j} \exp \left(p_{j} x_{1}+i \alpha x_{2}\right) d \alpha
\end{aligned}
$$

where $D_{j}$ 's $(j=3,4)$ can be obtained using one of the equilibrium equations in $(2.38 \mathrm{a}-\mathrm{b})$ and index $j$ is selected based on the roots $p_{j}$ 's. Keep in mind that terms related ( $j=1,2$ ) vanish in stress and displacement fields given by equations (2.40a-e) due to the regularity conditions in equations $(2.1 \mathrm{~h}-\mathrm{k})$. Using the following system of linear equations, $b_{3}$ and $b_{4}$ can be found.

$$
\begin{aligned}
& D_{j}=\frac{-C_{11} p_{j}^{2}+\frac{C_{66}}{2} \alpha^{2}}{\left(C_{12}+\frac{C_{66}}{2}\right) i \alpha p} \\
& \left\{b_{j}\right\}=-Y_{3}{ }^{-1} Y_{1} Y_{2}, \quad j=3,4 \\
& \sigma_{x_{1} x_{1}}^{(h)}\left(x_{1}=0, x_{2}\right)+W_{1}\left[\begin{array}{l}
\sigma_{X X}^{(i)}(X, Y) \\
\sigma_{Y Y}^{(i)}(X, Y) \\
\sigma_{X Y}^{(i)}(X, Y)
\end{array}\right]=0
\end{aligned}
$$




$$
\begin{aligned}
& \sigma_{x_{1} x_{2}}^{(h)}\left(x_{1}=0, x_{2}\right)+W_{2}\left[\begin{array}{l}
\sigma_{X X}^{(i)}(X, Y) \\
\sigma_{Y Y}^{(i)}(X, Y) \\
\sigma_{X Y}^{(i)}(X, Y)
\end{array}\right]=0 \\
& W_{1}=\left[\begin{array}{lll}
\cos ^{2}(\theta) & \sin ^{2}(\theta) & \sin (2 \theta)
\end{array}\right] \\
& W_{2}=\left[\begin{array}{lll}
-\cos (\theta) \sin (\theta) & \cos (\theta) \sin (\theta) & \cos (2 \theta)
\end{array}\right] \\
& \left\{\begin{array}{l}
x_{1} \\
x_{2}
\end{array}\right\}=\left[\begin{array}{rr}
\cos (\theta) & \sin (\theta) \\
-\sin (\theta) & \cos (\theta)
\end{array}\right]\left\{\begin{array}{l}
X \\
Y
\end{array}\right\}
\end{aligned}
$$

If $x_{1}=0$, then

$$
X=-\sin (\theta) x_{2} \quad Y=\cos (\theta) x_{2}
$$

Note that, the relations in (2.42f) and (2.42g) are used instead of $X$ and $Y$ in equation $(2.41 b)$. The equation in $(2.41 b)$ is rearranged in the matrix form.

$$
\left.\begin{array}{l}
Y_{1}=\left[\begin{array}{ccc}
\cos ^{2}(\theta) & \sin ^{2}(\theta) & \sin (2 \theta) \\
-\cos (\theta) \sin (\theta) & \cos (\theta) \sin (\theta) & \cos (2 \theta)
\end{array}\right] \\
\frac{1}{2 \pi} \int_{-\infty}^{\infty}\left[\begin{array}{c}
\int_{0}^{\infty} \sum_{j=3}^{4} S_{11} c_{j} \exp \left(n_{j} Y+i \omega X\right) \exp \left(-i \alpha x_{2}\right) d \alpha d \omega \\
+\int_{-\infty}^{0} \sum_{j=1}^{2} S_{11} c_{j} \exp \left(n_{j} Y+i \omega X\right) \exp \left(-i \alpha x_{2}\right) d \alpha d \omega
\end{array}\right] \\
\frac{1}{2 \pi} \int_{-\infty}^{\infty}\left[\begin{array}{c}
\int_{0}^{\infty} \sum_{j=3}^{4} S_{21} c_{j} \exp \left(n_{j} Y+i \omega X\right) \exp \left(-i \alpha x_{2}\right) d \alpha d \omega \\
+\int_{-\infty}^{0} \sum_{j=1}^{2} S_{21} c_{j} \exp \left(n_{j} Y+i \omega X\right) \exp \left(-i \alpha x_{2}\right) d \alpha d \omega
\end{array}\right] \\
\frac{1}{2 \pi} \int_{-\infty}^{\infty}\left[\begin{array}{l}
\int_{0}^{\infty} \sum_{j=3}^{4} S_{61} c_{j} \exp \left(n_{j} Y+i \omega X\right) \exp \left(-i \alpha x_{2}\right) d \alpha d \omega \\
+\int_{-\infty}^{0} \sum_{j=1}^{2} S_{61} c_{j} \exp \left(n_{j} Y+i \omega X\right) \exp \left(-i \alpha x_{2}\right) d \alpha d \omega
\end{array}\right]
\end{array}\right]
$$


$Y_{3}=\left[\begin{array}{ll}C_{11} p_{3}+C_{12} i \alpha D_{3} & C_{11} p_{4}+C_{12} i \alpha D_{4} \\ \frac{C_{66}}{2}\left(i \alpha+D_{3} p_{3}\right) & \frac{C_{66}}{2}\left(i \alpha+D_{4} p_{4}\right)\end{array}\right]$

The solution of equation (2.39) gives four real $p$ values. Two of them are with positive real parts and two of them are with negative real parts.

$$
\begin{array}{lllll}
p_{1}=q_{1}|\alpha| & p_{2}=q_{2}|\alpha| & \mathfrak{R}\left(p_{1}\right)>0 & \mathfrak{R}\left(p_{2}\right)>0 & (2.43 \mathrm{~d}, \mathrm{e}) \\
p_{3}=-q_{1}|\alpha| & p_{4}=-q_{2}|\alpha| & \mathfrak{R}\left(p_{3}\right)<0 & \Re\left(p_{4}\right)<0 & (2.43 \mathrm{f}, \mathrm{g})
\end{array}
$$

The inner integrals in equation (2.43b) are evaluated in MAPLE in the closed form. Residue Theorem is applied in order to evaluate the outer integrals in equation (2.43b) in the closed form. Detailed information about Residue Theorem can be found in Greenberg [38], Kreyszig [39] and Hildebrand [40]. Firstly, an integration contour $\Gamma$ like the one in Figure 2.10 is selected in the complex plane. Let a function $F(\omega, \alpha)$ be an analytical function except for finite number of singular points inside this contour. Then, Residue Theorem states the equation (2.44b).

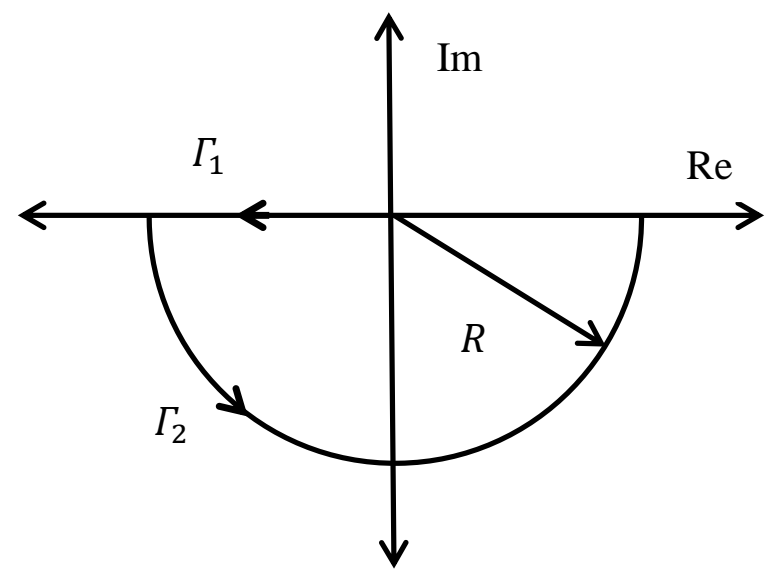

Figure 2.10 The Contour for Evaluation of Integral 


$$
\begin{aligned}
& \int_{\Gamma} F(\omega, \alpha) \exp (-i \omega t) d \omega=\int_{\Gamma_{1}} F(\omega, \alpha) \exp (-i \omega t) d \omega \\
& +\int_{\Gamma_{2}} F(\omega, \alpha) \exp (-i \omega t) d \omega \\
& \int_{\Gamma} F(\omega, \alpha) \exp (-i \omega t) d \omega \\
& =2 \pi i \sum_{k=1}^{n}(\text { Residues of the integrand in } \Gamma)
\end{aligned}
$$

Taking the limits as $R \rightarrow \infty \int_{\Gamma_{2}} F(\omega, \alpha) \exp (-i \omega t) d \omega \rightarrow 0$

For type 1 orthotropic material, the integral in (2.44c) always approach to zero as $R$ tends to infinity [2]. Lastly, the outer integrals in (2.43b) are evaluated by using equation $(2.44 \mathrm{e})$.

$$
\begin{aligned}
& \int_{-\infty}^{\infty} F(\omega, \alpha) \exp (-i \omega t) d \omega \\
& \quad=-2 \pi i \sum_{k=1}^{n} \begin{array}{c}
\text { Residues of the integrand in the } \\
\text { lower half complex plane })
\end{array} \\
& \int_{-\infty}^{\infty} F(\omega, \alpha) \exp (-i \omega t) d \omega \\
& =-2 \pi i\left(\begin{array}{r}
\lim _{\omega \rightarrow \omega_{3}}\left(\omega-\omega_{3}\right) F(\omega, \alpha) \exp (-i \omega t) \\
+\lim _{\omega \rightarrow \omega_{4}}\left(\omega-\omega_{4}\right) F(\omega, \alpha) \exp (-i \omega t)
\end{array}\right)
\end{aligned}
$$

The denominators of the kernels of the outer integrals in (2.43b) give four complex roots which make them singular in the following form.

$$
\omega_{1}=q_{7} i|\alpha|-q_{8} \alpha \quad \mathfrak{I}\left(\omega_{1}\right)>0
$$




$$
\begin{aligned}
& \omega_{2}=q_{9} i|\alpha|-q_{10} \alpha \quad \mathfrak{I}\left(\omega_{2}\right)>0 \\
& \omega_{3}=-q_{7} i|\alpha|-q_{8} \alpha \quad \mathfrak{I}\left(\omega_{3}\right)<0 \\
& \omega_{4}=-q_{9} i|\alpha|-q_{10} \alpha \quad \mathfrak{I}\left(\omega_{4}\right)<0
\end{aligned}
$$

where $q_{7}, q_{8}, q_{9}$ and $q_{10}$ are positive real numbers and lengthy functions of material parameters and crack angle. From those $\omega_{n}$ 's, only the ones in the lower complex half plane with negative imaginary parts $\left(\omega_{3}\right.$ and $\left.\omega_{4}\right)$ are needed for Residue Theorem. Now, $b_{3}$ and $b_{4}$ are found from two linear equations in (2.41b) and they are substituted to the related equations in (2.40a-e).

The infinite integrals in equations (2.40a-e) are evaluated in MAPLE. Stresses for the half plane solution are transformed to $X-Y$ coordinate system. In the end, the solution of crack problem is completed with the summation of displacement and stress equations.

$$
\begin{gathered}
\left\{\begin{array}{l}
U(X, Y)\} \\
V(X, Y)
\end{array}\right\}=\left\{\begin{array}{l}
U^{(i)}(X, Y) \\
V^{(i)}(X, Y)
\end{array}\right\}+\left[\begin{array}{rr}
\cos (\theta) & -\sin (\theta) \\
\sin (\theta) & \cos (\theta)
\end{array}\right]\left\{\begin{array}{l}
U^{(h)}\left(x_{1}, x_{2}\right) \\
V^{(h)}\left(x_{1}, x_{2}\right)
\end{array}\right\} \\
\left\{\begin{array}{l}
\sigma_{X X}^{\text {crack }}(X, Y) \\
\sigma_{Y Y}^{\text {crack }}(X, Y) \\
\sigma_{X Y}^{\text {crack }}(X, Y)
\end{array}\right\}=\left\{\begin{array}{l}
\sigma_{X X}{ }^{(i)}(X, Y) \\
\sigma_{Y Y}{ }^{(i)}(X, Y) \\
\sigma_{X Y}{ }^{(i)}(X, Y)
\end{array}\right\}+T\left\{\begin{array}{l}
\sigma_{x_{1} x_{1}}{ }^{(h)}\left(x_{1}, x_{2}\right) \\
\sigma_{x_{2} x_{2}}{ }^{(h)}\left(x_{1}, x_{2}\right) \\
\sigma_{x_{1} x_{2}}{ }^{(h)}\left(x_{1}, x_{2}\right)
\end{array}\right\} \\
T=\left[\begin{array}{ccc}
\cos ^{2}(\theta) & \sin ^{2}(\theta) & -\sin (2 \theta) \\
\sin ^{2}(\theta) & \cos ^{2}(\theta) & \sin (2 \theta) \\
\cos (\theta) \sin (\theta) & -\cos (\theta) \sin (\theta) & \cos (2 \theta)
\end{array}\right]
\end{gathered}
$$

\subsection{The Solution of Singular Integral Equations}

Using the boundary conditions in (2.1f) and (2.1g) and the relation in (2.46b), two singular integral equations are written after lengthy manipulations on the stresses given for infinite and half plane problems. 


$$
\begin{array}{cl}
\lambda_{1} \int_{0}^{d} \frac{f_{1}(t)}{t-X} d t+\int_{0}^{d} h_{11}(X, t) f_{1}(t) d t+\lambda_{2} \int_{0}^{d} \frac{f_{2}(t)}{t-X} d t & \\
\quad+\int_{0}^{d} h_{12}(X, t) f_{2}(t) d t=-\int_{a}^{b} h_{13}(X, t) \sigma(t) d t & \\
\lambda_{3} \int_{0}^{d} \frac{f_{1}(t)}{t-X} d t+\int_{0}^{d} h_{21}(X, t) f_{1}(t) d t+\lambda_{4} \int_{0}^{d} \frac{f_{2}(t)}{t-X} d t & 0<X<d \\
\quad+\int_{0}^{d} h_{22}(X, t) f_{2}(t) d t=-\int_{a}^{b} h_{23}(X, t) \sigma(t) d t &
\end{array}
$$

In these equations, the terms containing ' $t-X$ ' in the denominator are called as Cauchy kernels and the others are called as Fredholm kernels. Cauchy kernels are stemmed from infinite plane solution while Fredholm kernels are originated from half plane solution. $\lambda_{1}, \lambda_{2}, \lambda_{3}$ and $\lambda_{4}$ in equations (2.47a-b) are constants and lengthy functions of material parameters and crack angle. Note that the denominators of Cauchy kernels become zero in the given interval for $t=X$. Thus, these terms should be evaluated in closed form with the relation in Appendix B. On the other hand, singular points are not observed for Fredholm kernels. Hence, they can be evaluated using any quadrature method with a suitable weight function. Unfortunately, deriving parametric relations of the constant numbers $q_{3}, q_{4}, q_{5}, q_{6}$ in equations (2.35a-d), $q_{7}, q_{8}, q_{9}, q_{10}$ in equations (2.45a-d), $\lambda_{1}, \lambda_{2}, \lambda_{3}, \lambda_{4}$ and the kernels of the crack problem $h_{11}(X, t)$, $h_{12}(X, t), h_{21}(X, t)$ and $h_{22}(X, t)$ in equations $(2.47 \mathrm{a}-\mathrm{b})$ is not possible in terms of material parameters and crack angle because of too many independent terms involved in the problem. On the other hand, when numerical values are inserted to the material parameters, mentioned values and kernels can be calculated easily through asymptotic analysis in MAPLE.

The strengths of singularities of the unknown functions $f_{1}(t)$ and $f_{2}(t)$ at the end points of a surface crack are found in many studies by using function-theoretic 
method (For instance, Dag [1] and Sarikaya [2]). When function-theoretic method is applied to the singular integral equations, it is seen that they both have inverse square root singularity at the crack tip $X=d$ because of Cauchy kernels. However, there is no singularity at the other end point $X=0$. Hence, the unknown functions can be written in the following form.

$$
f_{1}(t)=\frac{F_{1}(t)}{(d-t)^{0.5}} \quad f_{2}(t)=\frac{F_{2}(t)}{(d-t)^{0.5}}
$$

In order to solve this system of singular integral equations, an expansioncollocation method is applied. The unknown crack surface displacement derivative functions and the intervals of variables $X$ and $t$ are normalized.

$t=\frac{d}{2} r+\frac{d}{2} \quad t=\frac{b-a}{2} \tilde{r}+\frac{b+a}{2}$

$X=\frac{d}{2} s_{1}+\frac{d}{2}$

$\Phi_{i}(r)=\frac{f_{i}\left(\frac{d}{2} r+\frac{d}{2}\right)}{(P /(b-a))}, \quad i=1,2$

$\Phi_{i}(r)=(1-r)^{-0.5} \sum_{n=0}^{\infty} A_{i n} P_{n}^{(-0.5,0)}(r), \quad i=1,2$

The normalized unknown functions are expanded as the series of Jacobi Polynomials.

$$
\begin{array}{lll}
\lambda_{1} \int_{-1}^{1} \frac{\Phi_{1}(r)}{r-s_{1}} d r+\int_{-1}^{1} H_{11}\left(s_{1}, r\right) \Phi_{1}(r) d r+\lambda_{2} \int_{-1}^{1} \frac{\Phi_{2}(r)}{r-s_{1}} d r & \\
\quad+\int_{-1}^{1} H_{12}\left(s_{1}, r\right) \Phi_{2}(r) d r=-\int_{-1}^{1} H_{13}\left(s_{1}, \tilde{r}\right) \Phi(\tilde{r}) d \tilde{r} & -1<s_{1}<1
\end{array}
$$




$$
\begin{gathered}
\lambda_{3} \int_{-1}^{1} \frac{\Phi_{1}(r)}{r-s_{1}} d r+\int_{-1}^{1} H_{21}\left(s_{1}, r\right) \Phi_{1}(r) d r+\lambda_{4} \int_{-1}^{1} \frac{\Phi_{2}(r)}{r-s_{1}} d r \\
+\int_{-1}^{1} H_{22}\left(s_{1}, r\right) \Phi_{2}(r) d r=-\int_{-1}^{1} H_{23}\left(s_{1}, \tilde{r}\right) \Phi(\tilde{r}) d \tilde{r}
\end{gathered}
$$

The integrals with Cauchy kernels in (2.50a-b) are calculated in closed form by using the relation in Appendix B. On the other hand, the integrals with Fredholm kernels are evaluated numerically with Gauss-Jacobi Quadrature Method.

$$
\begin{array}{lr}
\sum_{n=0}^{N} R_{11}\left(s_{1}\right) A_{1 n}+\sum_{n=0}^{N} R_{12}\left(s_{1}\right) A_{2 n}=R_{13}\left(s_{1}\right), & -1<s_{1}<1 \\
\sum_{n=0}^{N} R_{21}\left(s_{1}\right) A_{1 n}+\sum_{n=0}^{N} R_{22}\left(s_{1}\right) A_{2 n}=R_{23}\left(s_{1}\right), & -1<s_{1}<1 \\
s_{1 i}=\cos \left(\frac{\pi(2 i-1)}{2(N+1)}\right), & i=1, \ldots, N+1
\end{array}
$$

As the collocation points, the roots of the Chebyshev Polynomials of the first kind in equation $(2.51 \mathrm{c})$ are used. By inserting the collocation points into equations (2.51a-b), a linear system of equations is obtained and solved for the unknown coefficients of expansion. $\mathrm{N}$ is selected as 15 in (2.51a-c) for accuracy. Therefore, a system of 32 equations\&unknowns are solved.

In order to link the coefficients of expansions of unknown functions to the stress intensity factors, the following regulations are done. Since the denominator of Cauchy kernels approaches to zero at $X=d$, the dominant stress terms near $X \rightarrow d$ are written as follows:

$$
\sigma_{Y Y}(X, Y=0) \cong \lambda_{1} \int_{0}^{d} \frac{f_{1}(t)}{t-X} d t+\lambda_{2} \int_{0}^{d} \frac{f_{2}(t)}{t-X} d t
$$


$\sigma_{X Y}(X, Y=0) \cong \lambda_{3} \int_{0}^{d} \frac{f_{1}(t)}{t-X} d t+\lambda_{4} \int_{0}^{d} \frac{f_{2}(t)}{t-X} d t$

The following equations are obtained by inserting equations given by (2.48a-b) into (2.52a-b) and evaluating the integrals in MAPLE.

$$
\begin{aligned}
& \sigma_{Y Y}(X, Y=0) \cong \lambda_{1} \pi F_{1}(d)(X-d)^{-0.5}+\lambda_{2} \pi F_{2}(d)(X-d)^{-0.5} \\
& \sigma_{X Y}(X, Y=0) \cong \lambda_{3} \pi F_{1}(d)(X-d)^{-0.5}+\lambda_{4} \pi F_{2}(d)(X-d)^{-0.5}
\end{aligned}
$$

The definitions of mixed mode stress intensity factors at the crack tip are given as follows:

$k_{1}=\lim _{X \rightarrow d^{+}} \sqrt{2(X-d)} \sigma_{Y Y}(X, 0)$
$k_{2}=\lim _{X \rightarrow d^{+}} \sqrt{2(X-d)} \sigma_{X Y}(X, 0)$

The dominant stress terms are inserted to $(2.54 \mathrm{a}-\mathrm{b})$ and the relations are simplified to normalized mixed mode stress intensity factors.

$$
\begin{aligned}
& k_{1}=-\lim _{X \rightarrow d^{-}} \sqrt{2(d-X)}\left(\frac{F_{1}(r) \lambda_{1} \pi}{\sqrt{1-r}}+\frac{F_{2}(r) \lambda_{2} \pi}{\sqrt{1-r}}\right) \\
& k_{2}=-\lim _{X \rightarrow d^{-}} \sqrt{2(d-X)}\left(\frac{F_{1}(r) \lambda_{3} \pi}{\sqrt{1-r}}+\frac{F_{2}(r) \lambda_{4} \pi}{\sqrt{1-r}}\right) \\
& \frac{k_{1} \sqrt{d}}{P}=-\frac{d \pi}{(b-a)}\left[\lambda_{1} \sum_{n=0}^{N} A_{1 n} P_{n}^{(-0.5,0)}(1)+\lambda_{2} \sum_{n=0}^{N} A_{2 n} P_{n}^{(-0.5,0)}(1)\right] \\
& \frac{k_{2} \sqrt{d}}{P}=-\frac{d \pi}{(b-a)}\left[\lambda_{3} \sum_{n=0}^{N} A_{1 n} P_{n}^{(-0.5,0)}(1)+\lambda_{4} \sum_{n=0}^{N} A_{2 n} P_{n}^{(-0.5,0)}(1)\right]
\end{aligned}
$$


For fully open crack assumption, equations $(2.55 \mathrm{c}-\mathrm{d})$ give mode I and mode II stress intensity factors in the normalized form. In Chapter 3, the results with fully open crack assumption can be found.

\subsection{Implementation of Crack Closure}

In order to determine whether crack faces are in contact or not, the overall problem should be solved by fully open crack assumption. If mode I stress intensity factor obtained by the fully open crack assumption is positive, then fully open crack assumption is valid. However, if the results show otherwise, crack closure implementation is needed.

Unless the applied load is too complex, a single closure region between crack faces is expected. This assumption results in 4 closure modes in Figure 2.11. In order to determine correct closure mode shape, the problem is solved first using fully closed crack assumption ( Liu et al [41] ). Depending on contact pressure distribution between crack faces, the suitable closure mode can be selected easily. If contact pressure distribution is negative (compression) for all points along the crack line, then fully closed crack assumption is correct. On the other hand, the crack parts with positive contact pressure are not in contact.

For instance, when the crack is under the effect of bending load, crack mouth closure is observed for $\theta=10$ degrees. On the other hand, middle part of the crack is closed for a crack with $\theta=45$ degrees under bending load as shown in Figure 2.12. It is also found from further analyses that punch indentation always results in crack tip or complete (full length) closure modes. (For sample results, check Figure 2.13). Thus, crack tip and full length closure modes are examined for cracks loaded by punch indentation, while the results about crack mouth and middle part closure modes are presented for bending load only for the verification of the study. 


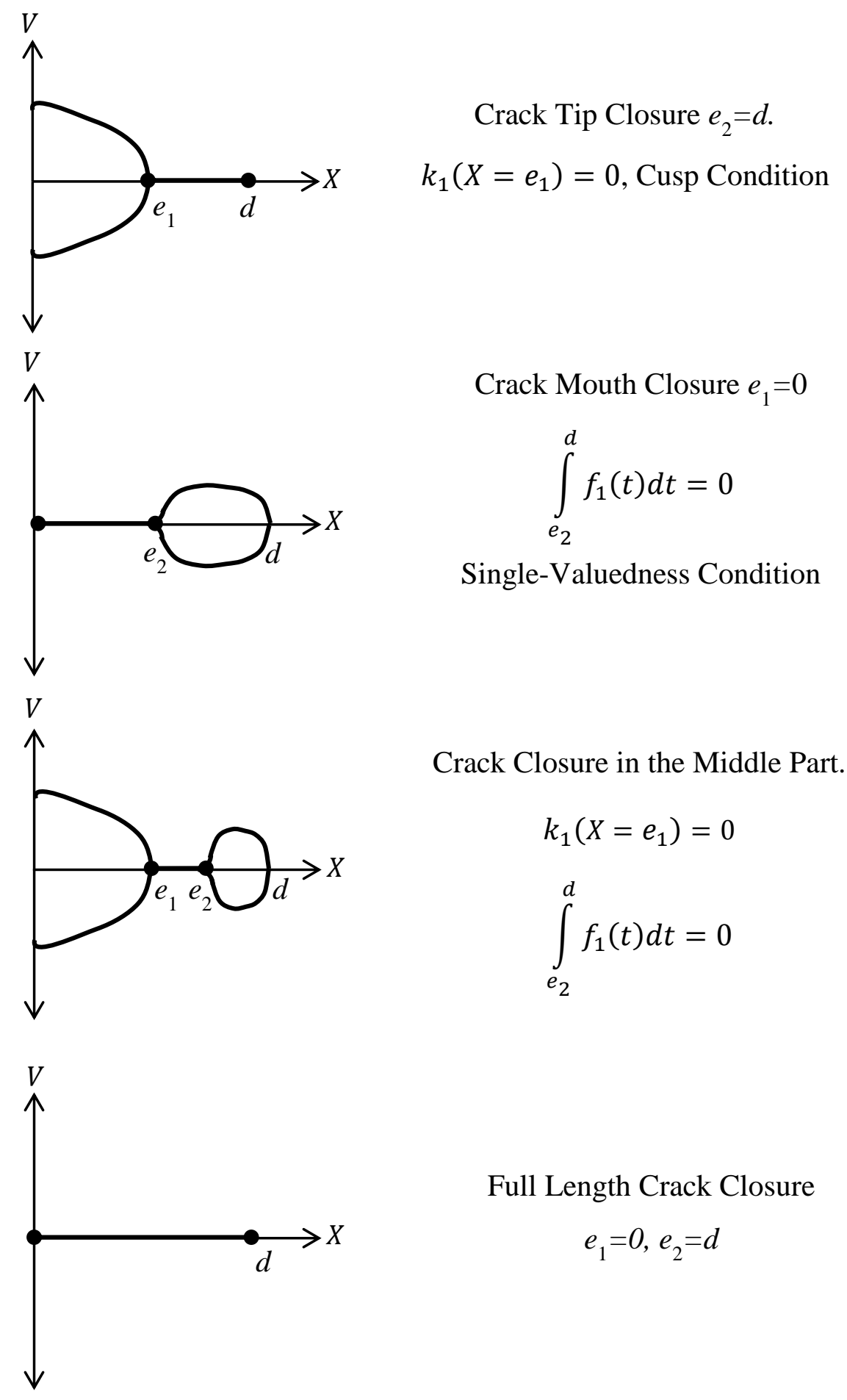

Figure 2.11 Crack Closure Mode Shapes 


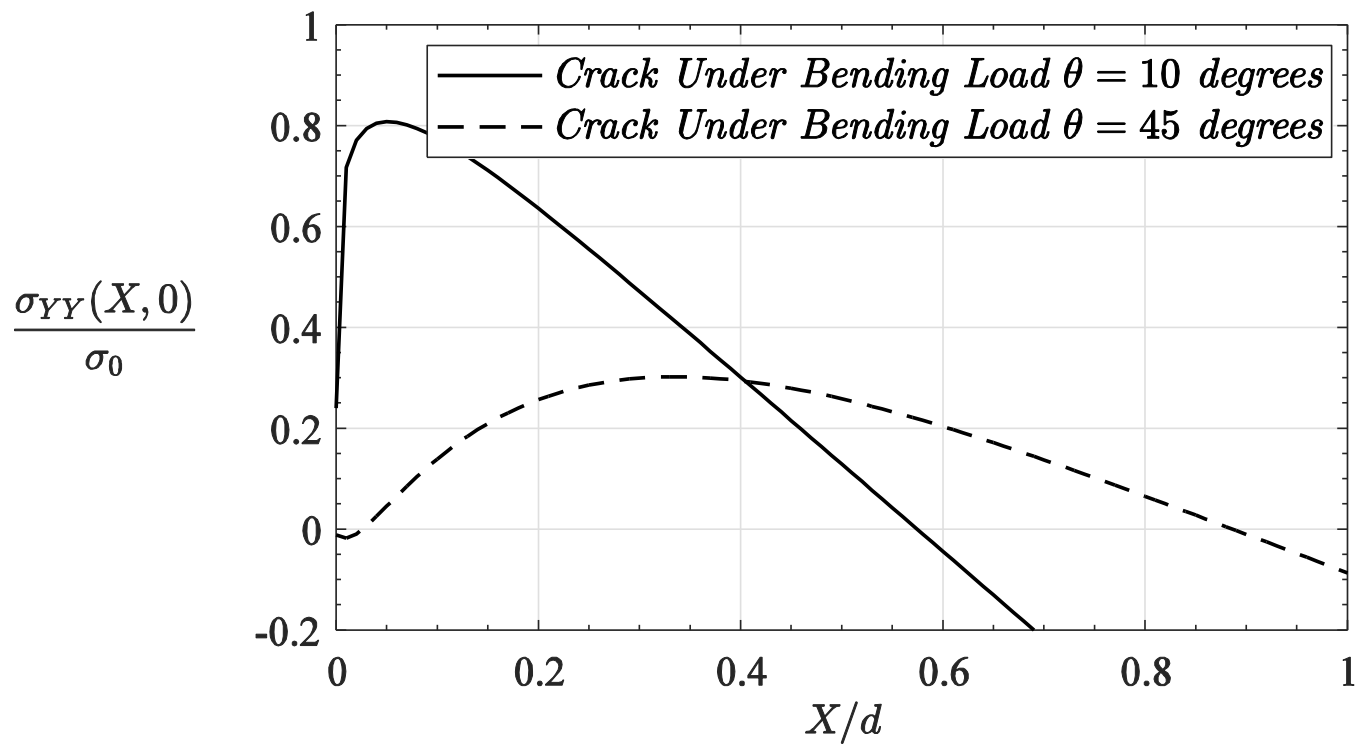

Figure 2.12 Sample Contact Pressure Distributions for Fully Closed Cracks Under Bending Load

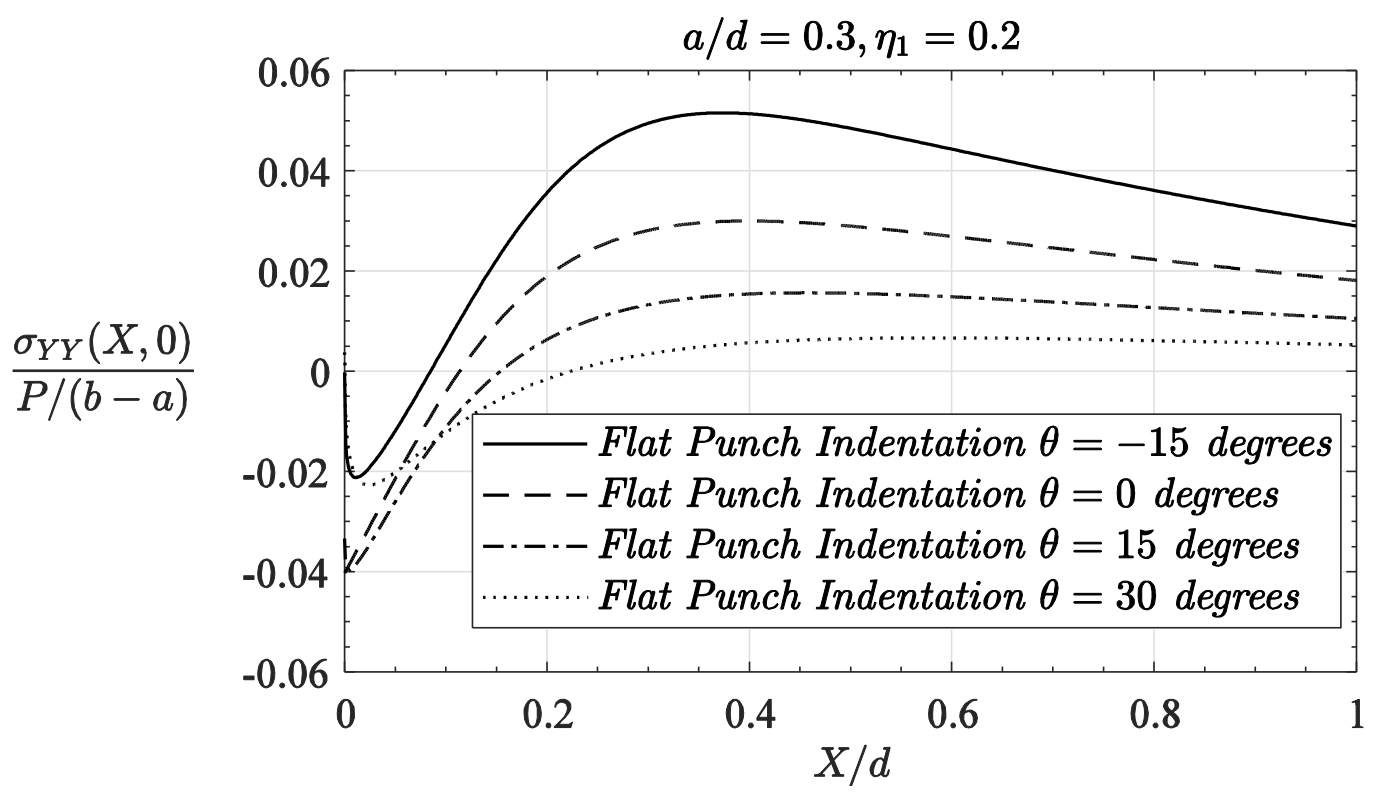

Figure 2.13 Sample Contact Pressure Distributions for Fully Closed Cracks Loaded by Punch Indentation 
In this section, sliding crack face contact boundary conditions are added to the system of singular integral equations. First, some of the boundary conditions given in the previous sections are updated. At the crack plane $(Y=0)$, normal displacements are zero in the closed region. Luckily, this boundary condition causes changes only in the intervals of singular integral equations and stress terms related to normal displacements while all kernels remain same. Normal and shear stresses exist for the closed portion of the crack because of the contact of the crack faces. The contact between crack faces is assumed to be flat. Coulomb's friction law is valid between normal and shear stresses at the closed crack faces. In Figure 2.11, there may be two additional unknowns $e_{1}$ and $e_{2}$ depending on the closure mode. Unlike the fully open crack assumption, additional boundary condition(s) in Figure 2.11 should also be satisfied this time which results in the iterative solution of system of singular integral equations. Therefore, $e_{1}$ and $e_{2}$ are iterated until the additional boundary conditions are satisfied.

For full length closure,

$$
\begin{array}{lc}
\sigma_{X Y}(X, 0)= \pm \eta_{2} \sigma_{Y Y}(X, 0), & 0<X<d \\
U\left(X, Y=0^{+}\right)=U\left(X, Y=0^{-}\right), & d<X \\
V\left(X, Y=0^{+}\right)=V\left(X, Y=0^{-}\right), & 0<X \\
U\left(X, Y=0^{+}\right) \neq U\left(X, Y=0^{-}\right), & 0<X<d
\end{array}
$$

For crack tip closure,

$$
\begin{array}{lc}
\sigma_{Y Y}(X, 0)=\sigma_{X Y}(X, 0)=0, & 0<X<e_{1} \\
\sigma_{X Y}(X, 0)= \pm \eta_{2} \sigma_{Y Y}(X, 0), & e_{1}<X<d \\
U\left(X, Y=0^{+}\right)=U\left(X, Y=0^{-}\right), & d<X \\
V\left(X, Y=0^{+}\right)=V\left(X, Y=0^{-}\right), & e_{1}<X \\
U\left(X, Y=0^{+}\right) \neq U\left(X, Y=0^{-}\right), & 0<X<d \\
V\left(X, Y=0^{+}\right) \neq V\left(X, Y=0^{-}\right), & 0<X<e_{1}
\end{array}
$$


Note that normal stress at the closed crack face is multiplied by $\mathrm{CoF}$ between crack faces ' $\eta_{2}$ ' and equated to shear stress in order to represent slipping of crack faces. The sign of friction between crack faces in (2.56a) and (2.57b) is applicable for both forward and backward slipping of crack faces. However, the sign of the friction between crack faces is arranged depending on the relative tangential movement of the crack faces.

\subsubsection{Full Length Closure Mode}

Among four closure modes, full length closure is the easiest closure mode to solve. Referring equations (2.56a-d), there is only one remaining singular integral equation.

$$
\begin{array}{ll}
\lambda_{4} \int_{0}^{d} \frac{f_{2}(t)}{t-X} d t+\int_{0}^{d} h_{22}(X, t) f_{2}(t) d t & 0<X<d \\
\pm \eta_{2}\left[\lambda_{2} \int_{0}^{d} \frac{f_{2}(t)}{t-X} d t+\int_{0}^{d} h_{12}(X, t) f_{2}(t) d t\right] & \\
=-\int_{a}^{b} h_{23}(X, t) \sigma(t) d t \pm \eta_{2} \int_{a}^{b} h_{13}(X, t) \sigma(t) d t &
\end{array}
$$

The unknown crack surface displacement derivative function and the intervals of variables $X$ and $t$ are normalized.

$$
\begin{aligned}
& t=\frac{b-a}{2} \tilde{r}+\frac{b+a}{2} \quad t=\frac{d}{2} r+\frac{d}{2} \quad X=\frac{d}{2} s_{1}+\frac{d}{2} \\
& f_{2}(t)=\frac{F_{2}(t)}{(d-t)^{0.5}} \quad \Phi_{2}(r)=\frac{f_{2}\left(\frac{d}{2} r+\frac{d}{2}\right)}{(P /(b-a))} \\
& \Phi_{2}(r)=(1-r)^{-0.5} \sum_{n=0}^{\infty} A_{2 n} P_{n}^{(-0.5,0)}(r)
\end{aligned}
$$


Normalization of this equation and other terms are done using (2.58b-g).

$$
\begin{aligned}
& \lambda_{4} \int_{-1}^{1} \frac{\Phi_{2}(r)}{r-s_{1}} d r+\int_{-1}^{1} H_{22}\left(s_{1}, r\right) \Phi_{2}(r) d r \\
& \pm \eta_{2}\left[\lambda_{2} \int_{-1}^{1} \frac{\Phi_{2}(r)}{r-s_{1}} d r+\int_{-1}^{1} H_{12}\left(s_{1}, r\right) \Phi_{2}(r) d r\right] \\
& =-\int_{-1}^{1} H_{23}\left(s_{1}, \tilde{r}\right) \Phi(\tilde{r}) d \tilde{r} \pm \eta_{2} \int_{-1}^{1} H_{13}\left(s_{1}, \tilde{r}\right) \Phi(\tilde{r}) d \tilde{r} \\
& s_{1 i}=\cos \left(\frac{\pi(2 i-1)}{2(N+1)}\right), \quad i=1 \ldots N+1 \\
& \sum_{n=0}^{N} R_{22}\left(s_{1}\right) A_{2 n}=R_{23}\left(s_{1}\right), \quad-1<s_{1}<1
\end{aligned}
$$

After the normalization, the integrals are evaluated by the relation given in Appendix B and suitable quadrature methods. A linear system of equations is obtained by inserting the collocations points given by (2.58i) into equation (2.58j). When the coefficients of expansion of the unknown function are found, normalized stress intensity factors and normal pressure distribution between crack faces can be obtained as follows:

$$
\begin{array}{lc}
\frac{k_{1} \sqrt{d}}{P}=0 & \\
\frac{k_{2} \sqrt{d}}{P}=-\frac{d \pi}{(b-a)}\left[\lambda_{4} \sum_{n=0}^{N} A_{2 n} P_{n}^{(-0.5,0)}(1) \pm \eta_{2} \lambda_{2} \sum_{n=0}^{N} A_{2 n} P_{n}^{(-0.5,0)}(1)\right] \\
\begin{array}{cc}
\sigma_{Y Y}(X, 0)=\lambda_{2} \int_{0}^{d} \frac{f_{2}(t)}{t-X} d t+\int_{0}^{d} h_{12}(X, t) f_{2}(t) d t & \text { Full Length } \\
+\int_{a}^{b} h_{13}(X, t) \sigma(t) d t & \text { Closure }
\end{array}
\end{array}
$$




\subsubsection{Crack Tip Closure Mode}

Referring equations (2.57a-f), two singular integral equations are written for crack tip closure mode by using Heaviside step function.

$$
\begin{array}{ll}
\lambda_{1} \int_{0}^{e_{1}} \frac{f_{1}(t)}{t-X} d t+\int_{0}^{e_{1}} h_{11}(X, t) f_{1}(t) d t+\lambda_{2} \int_{0}^{d} \frac{f_{2}(t)}{t-X} d t & \\
+\int_{0}^{d} h_{12}(X, t) f_{2}(t) d t=-\int_{a}^{b} h_{13}(X, t) \sigma(t) d t &
\end{array}
$$

$$
\begin{aligned}
& \lambda_{\mathbf{3}} \boldsymbol{H}\left(\boldsymbol{X}-\boldsymbol{e}_{\mathbf{1}}\right) \int_{\mathbf{0}}^{\boldsymbol{e}_{\mathbf{1}}} \frac{\boldsymbol{f}_{\mathbf{1}}(\boldsymbol{t})}{\boldsymbol{t}-\boldsymbol{X}} \boldsymbol{d} \boldsymbol{t}+\lambda_{3} H\left(e_{1}-X\right) \int_{0}^{e_{1}} \frac{f_{1}(t)}{t-X} d t \\
& +\int_{0}^{e_{1}} h_{21}(X, t) f_{1}(t) d t+\lambda_{4} \int_{0}^{d} \frac{f_{2}(t)}{t-X} d t \\
& +\int_{0}^{d} h_{22}(X, t) f_{2}(t) d t
\end{aligned}
$$$$
0<X<d(2.59 \mathrm{~b})
$$

$\pm \eta_{2} H\left(X-e_{1}\right)\left[\begin{array}{c}\lambda_{\mathbf{1}} \int_{\mathbf{0}}^{\boldsymbol{e}_{\mathbf{1}}} \frac{\boldsymbol{f}_{\mathbf{1}}(\boldsymbol{t})}{\boldsymbol{t}-\boldsymbol{X}} \boldsymbol{d} \boldsymbol{t}+\int_{0}^{e_{1}} h_{11}(X, t) f_{1}(t) d t \\ +\lambda_{2} \int_{0}^{d} \frac{f_{2}(t)}{t-X} d t+\int_{0}^{d} h_{12}(X, t) f_{2}(t) d t\end{array}\right]$

$=-\int_{a}^{b} h_{23}(X, t) \sigma(t) d t \pm \eta_{2} H\left(X-e_{1}\right) \int_{a}^{b} h_{13}(X, t) \sigma(t) d t$

It should be noted that bold written terms containing Heaviside step function become regular in equation (2.59b), although the kernels are same as Cauchy kernel [17]. In the next step, the unknown crack surface displacement derivative functions and the intervals of variables $X$ and $t$ are normalized. Similar 
expansion-collocation techniques are applied to two singular integral equations in (2.59a) and (2.59b).

$$
\begin{aligned}
& \lambda_{1} \int_{-1}^{1} \frac{\Phi_{1}(\bar{r})}{\bar{r}-\overline{s_{1}}} d \bar{r}+\int_{-1}^{1} H_{11}\left(\overline{s_{1}}, \bar{r}\right) \Phi_{1}(\bar{r}) d \bar{r} \\
& +\lambda_{2} \int_{-1}^{1} \frac{\Phi_{2}(r)}{r-V_{1}\left(\overline{s_{1}}\right)} d r+\int_{-1}^{1} H_{12}\left(\overline{s_{1}}, r\right) \Phi_{2}(r) d r \quad-1<\overline{s_{1}}<1 \\
& =-\int_{-1}^{1} H_{13}\left(\overline{s_{1}}, \tilde{r}\right) \Phi(\tilde{r}) d \tilde{r}
\end{aligned}
$$$$
\lambda_{3} H\left(V_{3}\left(s_{1}\right)-e_{1}\right) \int_{-1}^{1} \frac{\Phi_{1}(\bar{r})}{\bar{r}-V_{2}\left(s_{1}\right)} d \bar{r}
$$$$
+\lambda_{3} H\left(e_{1}-V_{3}\left(s_{1}\right)\right) \int_{-1}^{1} \frac{\Phi_{1}(\bar{r})}{\bar{r}-V_{2}\left(s_{1}\right)} d \bar{r}
$$$$
+\int_{-1}^{1} H_{21}\left(s_{1}, \bar{r}\right) \Phi_{1}(\bar{r}) d \bar{r}+\lambda_{4} \int_{-1}^{1} \frac{\Phi_{2}(r)}{r-s_{1}} d r
$$$$
+\int_{-1}^{1} H_{22}\left(s_{1}, r\right) \Phi_{2}(r) d r
$$$$
-1<s_{1}<1
$$

$$
\begin{aligned}
& \pm \eta_{2} H\left(V_{3}\left(s_{1}\right)-e_{1}\right) \\
& {\left[\begin{array}{c}
\left.\lambda_{\mathbf{1}} \int_{-\mathbf{1}}^{\mathbf{1}} \frac{\boldsymbol{\Phi}_{\mathbf{1}}(\overline{\boldsymbol{r}})}{\overline{\boldsymbol{r}}-\boldsymbol{V}_{\mathbf{2}}\left(\boldsymbol{s}_{\mathbf{1}}\right)} \boldsymbol{d} \overline{\boldsymbol{r}}+\int_{-1}^{1} H_{11}\left(s_{1}, \bar{r}\right) \Phi_{1}(\bar{r}) d \bar{r}\right] \\
+\lambda_{2} \int_{-1}^{1} \frac{\Phi_{2}(r)}{r-s_{1}} d r+\int_{-1}^{1} H_{12}\left(s_{1}, r\right) \Phi_{2}(r) d r
\end{array}\right]} \\
& =-\int_{-1}^{1} H_{23}\left(s_{1}, \tilde{r}\right) \Phi(\tilde{r}) d \tilde{r} \\
& \pm \eta_{2} H\left(V_{3}\left(s_{1}\right)-e_{1}\right) \int_{-1}^{1} H_{13}\left(s_{1}, \tilde{r}\right) \Phi(\tilde{r}) d \tilde{r}
\end{aligned}
$$




$$
\begin{aligned}
& V_{1}\left(\overline{s_{1}}\right)=\left[\frac{e_{1}}{d} \overline{s_{1}}+\frac{e_{1}}{d}-1\right] \\
& V_{2}\left(s_{1}\right)=\left[\frac{d}{e_{1}} s_{1}+\frac{d}{e_{1}}-1\right] \\
& V_{3}\left(s_{1}\right)=\left[\frac{d}{2} s_{1}+\frac{d}{2}\right]
\end{aligned}
$$

$$
X=\frac{e_{1}}{2} \overline{s_{1}}+\frac{e_{1}}{2} \quad X=\frac{d}{2} s_{1}+\frac{d}{2}
$$

$$
t=\frac{b-a}{2} \tilde{r}+\frac{b+a}{2}
$$

$$
t=\frac{e_{1}}{2} \bar{r}+\frac{e_{1}}{2} \quad t=\frac{d}{2} r+\frac{d}{2}
$$

$$
f_{1}(t)=\frac{F_{1}(t)}{\left(e_{1}-t\right)^{0.5}} \quad f_{2}(t)=\frac{F_{2}(t)}{(d-t)^{0.5}}
$$

$$
\Phi_{1}(\bar{r})=\frac{f_{1}\left(\frac{e_{1}}{2} \bar{r}+\frac{e_{1}}{2}\right)}{(P /(b-a))} \quad \Phi_{2}(r)=\frac{f_{2}\left(\frac{d}{2} r+\frac{d}{2}\right)}{(P /(b-a))}
$$

$\Phi_{1}(r)=(1-\bar{r})^{-0.5} \sum_{n=0}^{\infty} A_{1 n} P_{n}^{(-0.5,0)}(r)$

$\Phi_{2}(r)=(1-r)^{-0.5} \sum_{n=0}^{\infty} A_{2 n} P_{n}^{(-0.5,0)}(r)$

$$
\overline{s_{1 i}}=\cos \left(\frac{\pi(2 i-1)}{2(N+1)}\right), \quad s_{1 i}=\cos \left(\frac{\pi(2 i-1)}{2(N+1)}\right), i=1 . . N+1
$$

Normalization of these equations and other terms are done using (2.59h-r).After the normalization, the integrals are evaluated by the relation given in Appendix B and suitable quadrature methods. A non-linear system of equations is obtained by inserting the collocations points given by $(2.59 \mathrm{~s})$ and $(2.59 \mathrm{t})$ into equations $(2.59 \mathrm{c})$ and $(2.59 \mathrm{~d})$. Lastly, the closure point $e_{1}$ is iterated until the cusp condition given in Figure 2.11 is satisfied. $\left(k_{1}\left(X=e_{1}\right)=0\right)$. 


$$
\begin{aligned}
& \sum_{n=0}^{N} R_{11}\left(\overline{s_{1}}\right) A_{1 n}+\sum_{n=0}^{N} R_{12}\left(\overline{s_{1}}\right) A_{2 n}=R_{13}\left(\overline{s_{1}}\right), \quad-1<\overline{s_{1}}<1 \\
& \sum_{n=0}^{N} R_{21}\left(s_{1}\right) A_{1 n}+\sum_{n=0}^{N} R_{22}\left(s_{1}\right) A_{2 n}=R_{23}\left(s_{1}\right), \quad-1<s_{1}<1
\end{aligned}
$$

When the coefficients of expansions of the unknown functions are found, normalized SIF's and normal pressure distribution between crack faces can be obtained as follows:

$$
\begin{aligned}
& \frac{k_{1} \sqrt{d}}{P}=0 \\
& \frac{k_{1} \sqrt{e_{1}}}{P}=-\frac{e_{1} \pi}{(b-a)}\left[\lambda_{1} \sum_{n=0}^{N} A_{1 n} P_{n}^{(-0.5,0)}(1)\right]=0 \\
& \frac{k_{2} \sqrt{d}}{P}=-\frac{d \pi}{(b-a)}\left[\lambda_{4} \sum_{n=0}^{N} A_{2 n} P_{n}^{(-0.5,0)}(1) \pm \eta_{2} \lambda_{2} \sum_{n=0}^{N} A_{2 n} P_{n}^{(-0.5,0)}(1)\right] \\
& \sigma_{Y Y}(X, 0)=\lambda_{1} \int_{0}^{e_{1}} \frac{f_{1}(t)}{t-X} d t+\int_{0}^{e_{1}} h_{11}(X, t) f_{1}(t) d t \\
& +\lambda_{2} \int_{0}^{d} \frac{f_{2}(t)}{t-X} d t+\int_{0}^{d} h_{12}(X, t) f_{2}(t) d t \\
& \quad+\int_{a}^{b} h_{13}(X, t) \sigma(t) d t
\end{aligned}
$$

Eventually, crack tip closure problem is completed with normalized relative crack surface displacements by integrating equations (2.2a) and (2.2b).

$$
\frac{Q_{66} \Delta V(X)}{e_{1}(P /(b-a))}=\left(\frac{X}{e_{1}}-1\right) \int_{-1}^{1} \Phi_{1}\left(\frac{X}{e_{1}} r-r+\frac{X}{e_{1}}\right) d r \quad 0<X<e_{1}
$$


$\frac{Q_{66} \Delta U(X)}{d(P /(b-a))}=\left(\frac{X}{d}-1\right) \int_{-1}^{1} \Phi_{2}\left(\frac{X}{d} r-r+\frac{X}{d}\right) d r \quad 0<X<d$

Note that only crack tip and full length closure modes are within the scope of the present study. However, investigations of other crack closure modes are also presented by applying bending load for verification in the following sections.

For crack tip closure mode, $e_{1}$ is taken as $\mathrm{d}$ at the first attempt in the iterative solution. If the solution gives negative mode I stress intensity factor, $e_{1}$ is adjusted with bisection method. At the second attempt, $e_{1}$ is taken as $\mathrm{d} / 2$. If the solution still gives negative mode I stress intensity factor, $e_{1}$ becomes $\mathrm{d} / 4$. However, if positive mode I stress intensity factor is obtained, $e_{1}$ is equated to $3 \mathrm{~d} / 4$. This procedure continues until $e_{1}$ is squeezed in a suitable range. A similar algorithm is applied for crack mouth closure mode except for this time, $e_{2}$ is adjusted according to single-valuedness condition in Figure 2.11. Unfortunately, an algorithm cannot be developed for middle part closure problem since both $e_{1}$ and $e_{2}$ affect both cusp and single-valuedness conditions in Figure 2.11. Therefore, the values for $e_{1}$ and $e_{2}$ should be adjusted manually for middle part closure mode. 


\section{CHAPTER 3}

\section{NUMERICAL RESULTS}

For the numerical calculations, plasma sprayed alumina $\left(\mathrm{Al}_{2} \mathrm{O}_{3}\right)$ with the material properties in equations (3.1a-g) ( Sarikaya [2] ) is chosen. Due to its hardness, wear resistance and high temperature strength, plasma sprayed alumina is preferred as coatings in many engineering applications. The usage of plasma sprayed alumina as wear resistant coating can be encountered in textile machinery parts, automobile parts, engines, aircraft parts, computer systems (hard disk of a computer) and bioceramics (joint prosthetics). Besides, several elastic modulus ratios $\left(E_{2} / E_{1}\right.$ and $\left.E_{3} / E_{1}\right)$ are considered in order to investigate the influence of material properties on the results as long as Poisson's ratios of the material satisfy equations (3.1h-k). Figures, related to the effect of loading size crack length ratio $((b-a) / d)$ and crack angle $(\theta)$ variation, have also been presented. In addition to selecting punch indentation as loading, uniform tension and bending loads are also examined for verification. Depending on the iterative solution of singular integral equations, closed portions are calculated and illustrated in the related figures. Lastly, mixed mode stress intensity factors and normal pressure distributions at the closed crack faces are examined.

$$
\begin{aligned}
& E_{1}: 116.36 \mathrm{GPa} \quad E_{2}: 90.43 \mathrm{GPa} \quad \mu_{12}: 38.21 \mathrm{GPa} \quad(3.1 \mathrm{a}, \mathrm{b}, \mathrm{c}) \\
& v_{12}: 0.28 \quad v_{13}: 0.27 \quad v_{31}: 0.21 \quad v_{32}: 0.14 \quad(3.1 \mathrm{~d}, \mathrm{e}, \mathrm{f}, \mathrm{g}) \\
& 1-v_{12} v_{21}>0, \quad 1-v_{13} v_{31}>0, \quad 1-v_{23} v_{32}>0 \quad(3.1 \mathrm{~h}, \mathrm{i}, \mathrm{j}) \\
& 1-v_{12} v_{21}-v_{13} v_{31}-v_{23} v_{32}-2 v_{12} v_{23} v_{31}>0
\end{aligned}
$$


The inclined edge crack formulation in the present study is confirmed by Beghini et al [42]. Finite element method is applied by Beghini et al [42] in order to evaluate SIF's for an inclined edge crack in isotropic medium under uniform tension as illustrated in Figure 3.1. When orthotropic material constants are arranged as though the material is isotropic, the results in both studies agree quite well between $\theta=0-75$ degrees as shown in Figure 3.2. Besides, finite element results for SIF's related to inclined cracks in orthotropic medium are provided using Ansys by implementing pre-meshed crack analysis. A pre-meshed crack is a fracture tool in Ansys based on a previously-generated mesh and utilizes a node-based named selection to analyze SIF's. In both comparisons, a convergence trend is observed as shown in Figure 3.2. For those cases, singular integral equations are modified by changing the last terms in equations $(2.47 a-b)$ with uniform tension $-\cos ^{2}(\theta) \sigma_{0}$ and $-\sin (\theta) \cos (\theta) \sigma_{0}$, respectively. Thus, SIF's are given in the following form. Effect of $E_{2} / E_{1}$ ratio on mixed mode stress intensity factors for a crack under uniform tension is also given in Figure 3.3.

$$
\begin{aligned}
& k_{1}=F_{1} \sigma_{0} \sqrt{\pi d}, F_{1}=-\pi\left[\lambda_{1} \sum_{n=0}^{N} A_{1 n} P_{n}^{(-0.5,0)}(1)+\lambda_{2} \sum_{n=0}^{N} A_{2 n} P_{n}^{(-0.5,0)}(1)\right] \\
& k_{2}=F_{2} \sigma_{0} \sqrt{\pi d}, F_{2}=-\pi\left[\lambda_{3} \sum_{n=0}^{N} A_{1 n} P_{n}^{(-0.5,0)}(1)+\lambda_{4} \sum_{n=0}^{N} A_{2 n} P_{n}^{(-0.5,0)}(1)\right]
\end{aligned}
$$

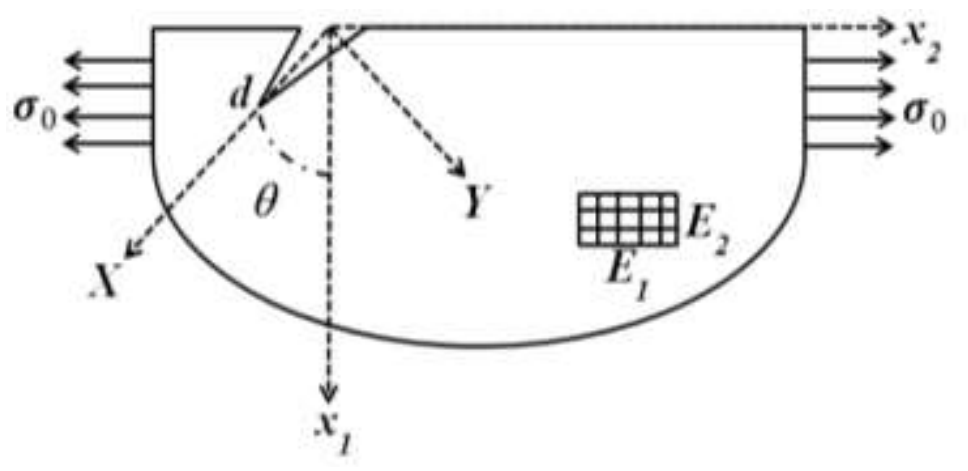

Figure 3.1 Representation of the Problem by Beghini et al [42] 

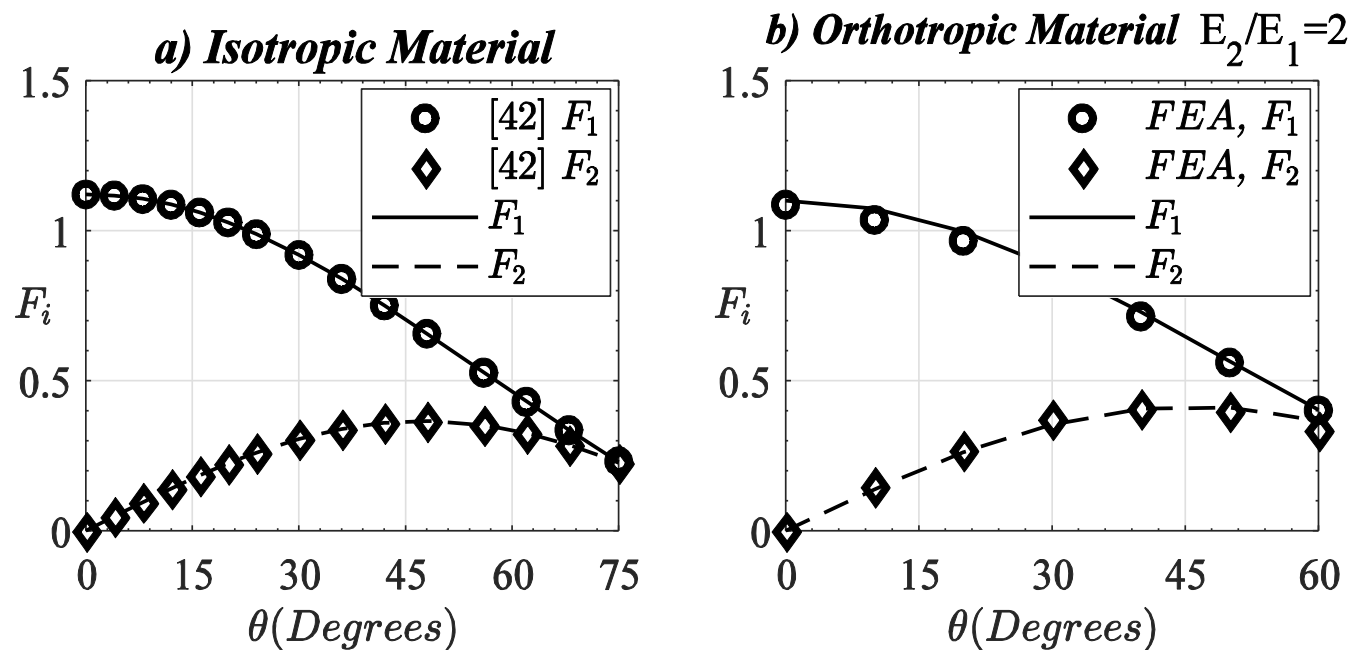

Figure 3.2 Comparison of SIF's for Inclined Edge Cracks in a) Isotropic Material b) Orthotropic Material under Uniform Tension.
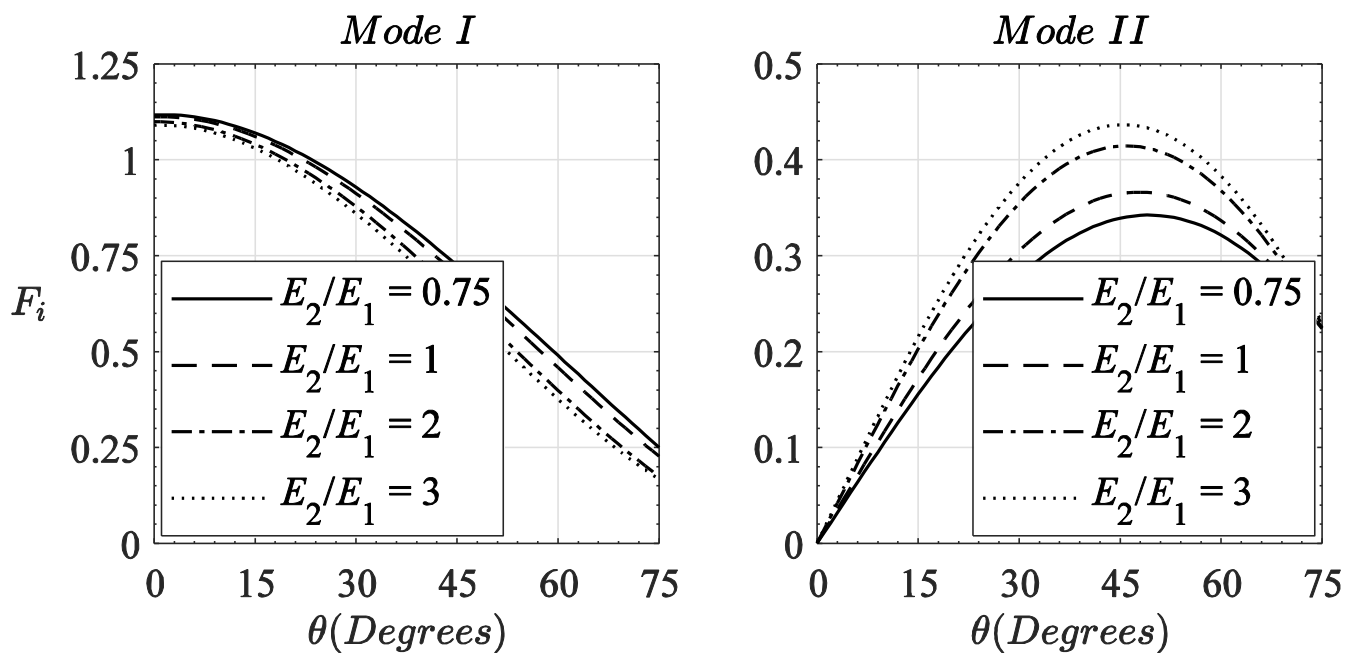

Figure 3.3 Effect of Elastic Modulus Ratio $E_{2} / E_{1}$ on Mixed Mode Stress Intensity Factors for Inclined Edge Crack in Orthotropic Half-Plane under Uniform Tension for Plane Strain Case. 
To verify the formulation of orthotropic material, the results with zero crack angles $(\theta=0)$ have been compared with the ones given by Sarikaya [2] for orthotropic half-plane of plasma sprayed alumina indented by flat, triangular and circular punches in Figure 3.4, Figure 3.5 and Figure 3.6, respectively. Since the coupled contact loadinglcrack problem is solved simultaneously by Sarikaya [2], small deviations may be expected for the results having low $a / d$ ratio (when the crack is placed in the closed vicinity of the loading region). The reason for this deviation is that the effect of the presence of the crack on the loading functions at the material surface is neglected in the present study. Hence, the loading functions alter in the results given by Sarikaya [2] when the starting point of the contact of the punch is very close to the crack. Nevertheless, excellent agreement has been observed between two studies for the results with high $a / d$ ratio.
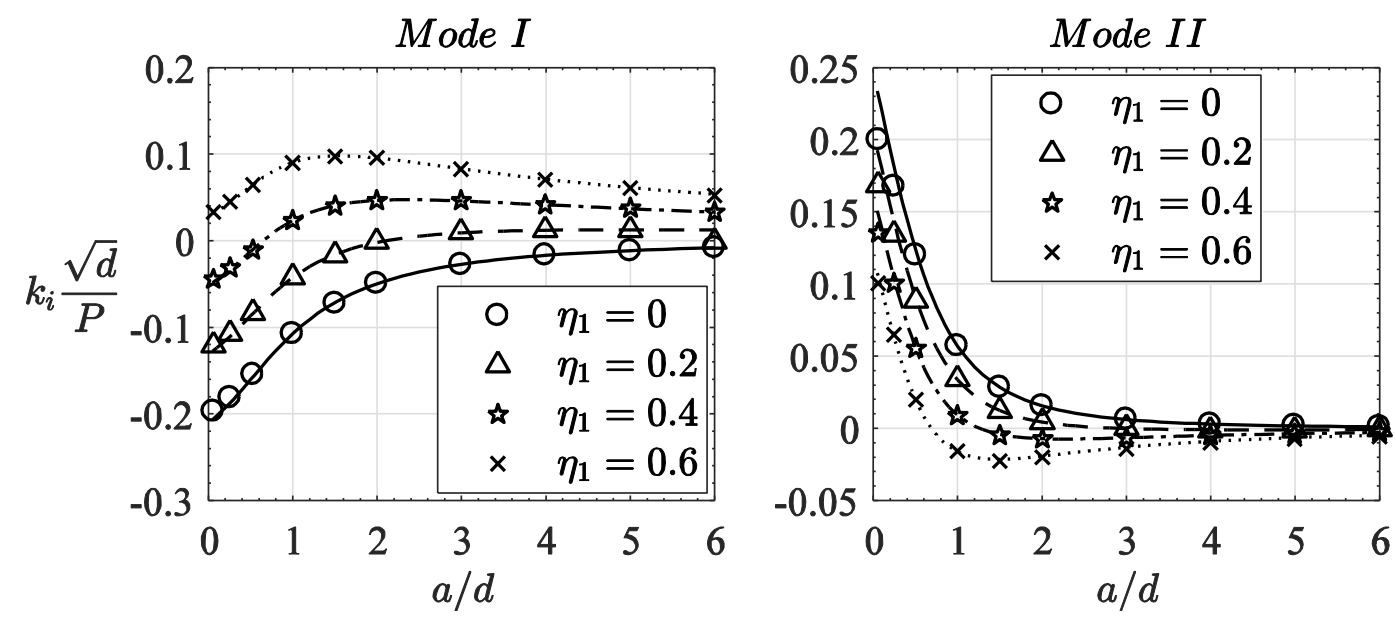

Figure 3.4 Comparison of Mixed Mode Stress Intensity Factors for Straight Edge Crack Provided by Sarikaya [2] (Markers) and Present Study (Lines) in Orthotropic Half-Plane of Plasma Sprayed Alumina Loaded by Flat Punch for Plane Strain Case and Fully Open Crack Assumption, $(b-a) / d=1$. 

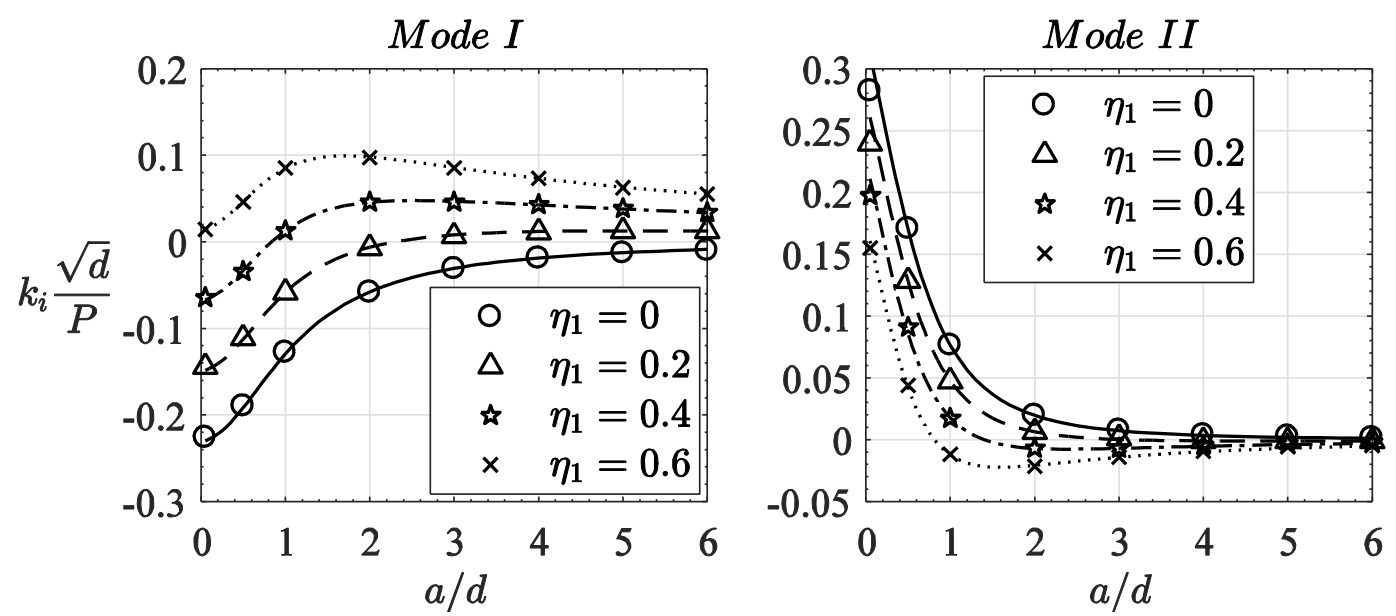

Figure 3.5 Comparison of Mixed Mode Stress Intensity Factors for Straight Edge Crack Provided by Sarikaya [2] (Markers) and Present Study (Lines) in Orthotropic Half-Plane of Plasma Sprayed Alumina Loaded by Triangular Punch for Plane Strain Case and Fully Open Crack Assumption, $(b-a) / d=1$.
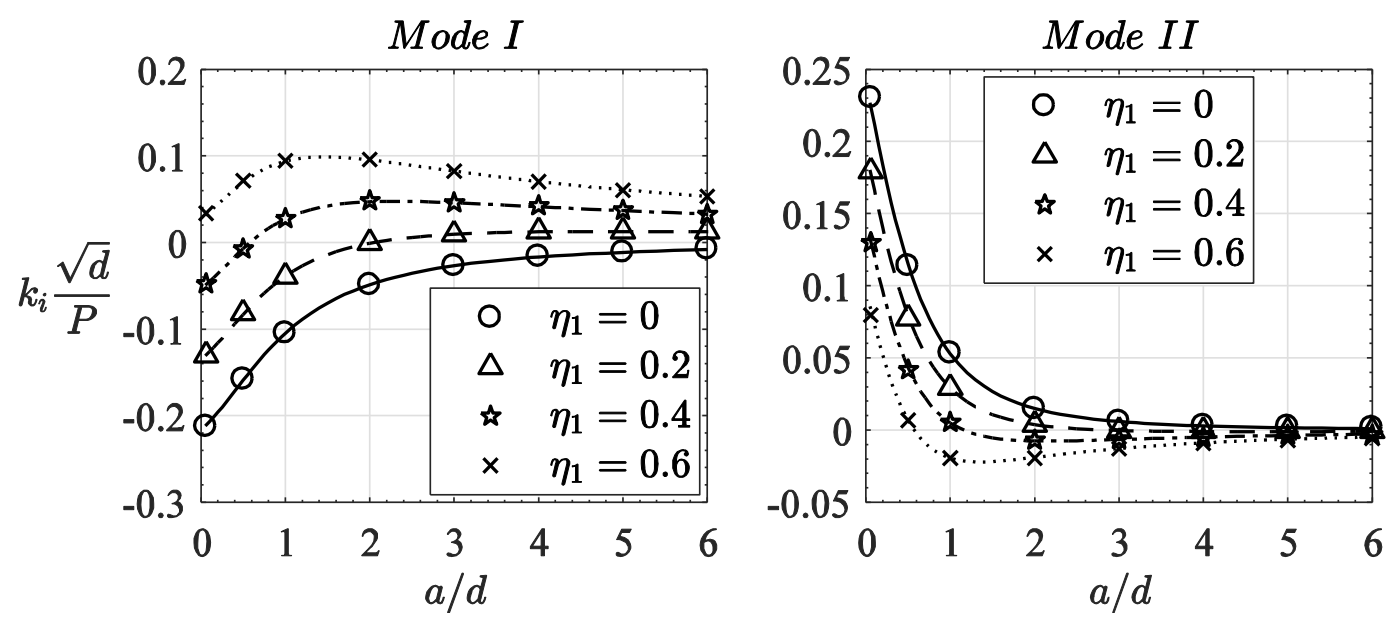

Figure 3.6 Comparison of Mixed Mode Stress Intensity Factors for Straight Edge Crack Provided by Sarikaya [2] (Markers) and Present Study (Lines) in Orthotropic Half-Plane of Plasma Sprayed Alumina Loaded by Circular Punch for Plane Strain Case and Fully Open Crack Assumption, $(b-a) / d=1$. 


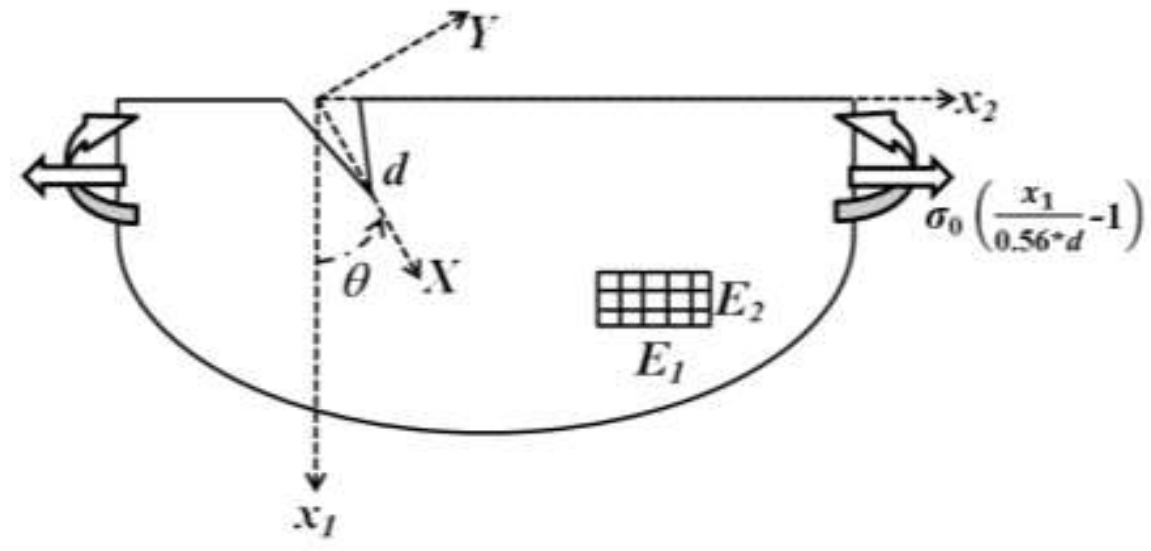

Figure 3.7 Representation of the Problem by Beghini et al [43]. (Inclined Edge Crack under Bending Load)

Last verification in this study is related with closed cracks. Beghini et al [43] examine SIF's for closed cracks in isotropic medium under bending load. The configuration of the problem is given in Figure 3.7. Stress intensity factors are given considering both weight function method and finite element method by both ignoring and adding the effects of crack closure. Beghini and co-workers report that when crack angle is between 0 and $\approx 35$ degrees, crack mouth closure is observed. Thus, transition to middle part closure mode starts about 35 degrees. If crack angle is further increased, middle part closure mode is detected until almost 52 degrees. If crack angle is taken greater than 52 degrees, crack tip closure is noticed. When the proposed method in this study is modified accordingly with the equations below, closure modes, mixed mode SIF's and contact pressure distributions seem consistent with the ones in Beghini et al [43] (Check Figure 3.8 and Figure 3.9).

$-\int_{a}^{b} h_{13}(X, t) \sigma(t) d t \rightarrow-\sigma_{0}\left(\frac{X \cos (\theta)}{0.56 d}-1\right)\left(\frac{1+\cos (2 \theta)}{2}\right)$ 
$-\int_{a}^{b} h_{23}(X, t) \sigma(t) d t »-\sigma_{0}\left(\frac{X \cos (\theta)}{0.56 d}-1\right)\left(\frac{\sin (-2 \theta)}{2}\right)$

SIF's for Fully Open Crack Assumption

$$
\begin{aligned}
& \frac{k_{1}}{\sigma_{0} \sqrt{d}}=-\pi^{1.5}\left[\lambda_{1} \sum_{n=0}^{N} A_{1 n} P_{n}^{(-0.5,0)}(1)+\lambda_{2} \sum_{n=0}^{N} A_{2 n} P_{n}^{(-0.5,0)}(1)\right] \\
& \frac{k_{2}}{\sigma_{0} \sqrt{d}}=-\pi^{1.5}\left[\lambda_{3} \sum_{n=0}^{N} A_{1 n} P_{n}^{(-0.5,0)}(1)+\lambda_{4} \sum_{n=0}^{N} A_{2 n} P_{n}^{(-0.5,0)}(1)\right]
\end{aligned}
$$

SIF's for Crack Mouth and Middle Part Closure Modes

$$
\begin{aligned}
\frac{k_{1}}{\sigma_{0} \sqrt{d}}=-\pi^{1.5} & {\left[\sqrt{2\left(\frac{1-e_{2}}{d}\right)} \lambda_{1} \sum_{n=0}^{N} A_{12 n} P_{n}^{(-0.5,0.5)}(1)\right.} \\
& \left.+\lambda_{2} \sum_{n=0}^{N} A_{2 n} P_{n}^{(-0.5,0)}(1)\right] \\
\frac{k_{2}}{\sigma_{0} \sqrt{d}}=-\pi^{1.5} & {\left[\sqrt{2\left(\frac{1-e_{2}}{d}\right)} \lambda_{3} \sum_{n=0}^{N} A_{12 n} P_{n}^{(-0.5,0.5)}(1)\right.} \\
& \left.+\lambda_{4} \sum_{n=0}^{N} A_{2 n} P_{n}^{(-0.5,0)}(1) \pm \eta_{2} \lambda_{2} \sum_{n=0}^{N} A_{2 n} P_{n}^{(-0.5,0)}(1)\right]
\end{aligned}
$$

Numerical results for flat, triangular and circular punch profiles are respectively provided for fully open crack assumption in Figure 3.10-Figure 3.20, Figure 3.21Figure 3.31 and Figure 3.32-Figure 3.42. The first notice about these results is that changing the punch profile causes significant changes only for low values of $a / d$. Because the pressure distribution between punch and material surface affects SIF's when the loading area is near the crack. Figure 3.10-Figure 3.12, Figure 3.21-Figure 3.23 and Figure 3.32-Figure 3.34 illustrate the relations between SIF's and crack angle and CoF at the material surface. 

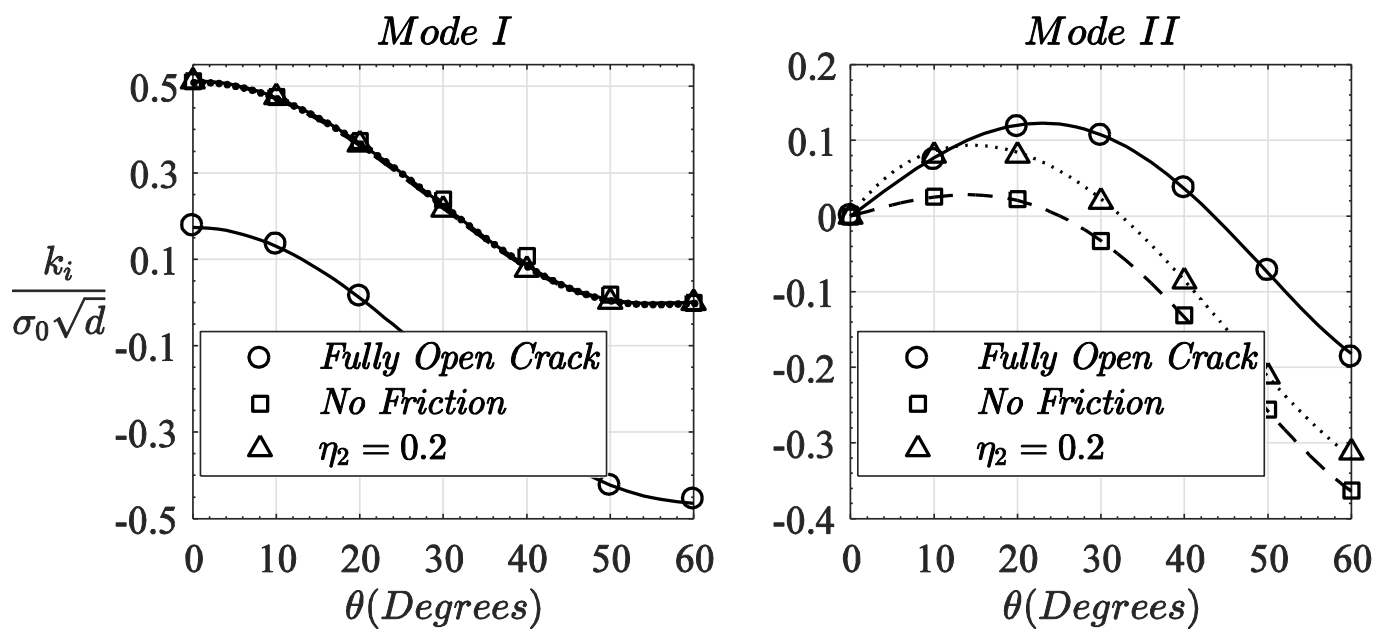

Figure 3.8 Comparison of SIF's by Beghini et al [43] (Markers) and Present Study (Lines) for Inclined Edge Crack under Bending Load. (Orthotropic Material Constants: $\mathrm{E}_{1}=116.37 \mathrm{GPa}, \mathrm{E}_{2}=116.35 \mathrm{GPa}, \mu_{12}=46.54 \mathrm{GPa}, \boldsymbol{v}=0.25$ )

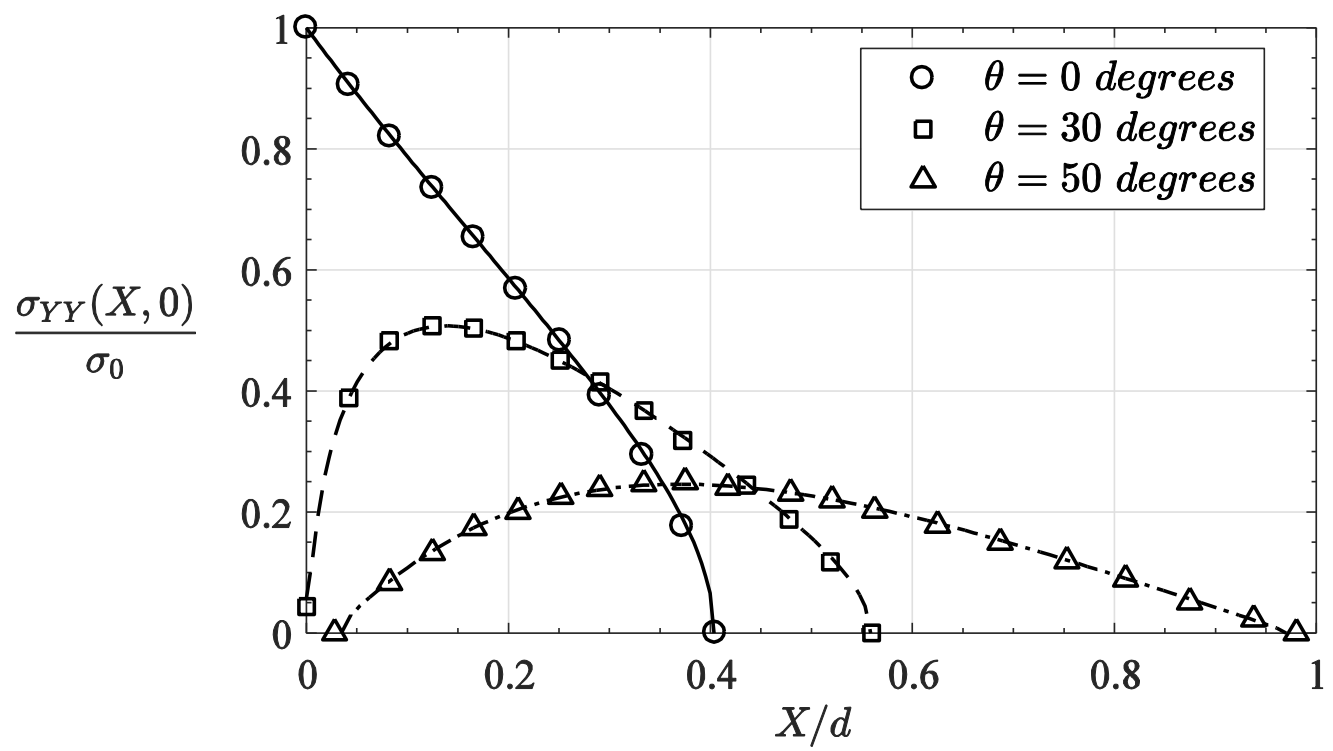

Figure 3.9 Comparison of Normal Contact Pressure Distributions by Beghini et al [43] (Markers) and Present Study (Lines) for Frictionless Contact. (Orthotropic Material Constants: $\mathrm{E}_{1}=116.37 \mathrm{GPa}, \mathrm{E}_{2}=116.35 \mathrm{GPa}, \mu_{12}=46.54 \mathrm{GPa}, \boldsymbol{v}=0.25$ ) 
The impact of $(b-a) / d$ ratio on the results is shown in Figure 3.13-Figure 3.14, Figure 3.24-Figure 3.25 and Figure 3.35-Figure 3.36 for crack angle $\theta=\pi / 12$. For small values of $a / d$, the influence of $(b-a) / d$ ratio is more apparent.

Another set of results in Figure 3.15-Figure 3.18, Figure 3.26-Figure 3.29 and Figure 3.37-Figure 3.40 are provided for three punch profiles to show the influence of elastic modulus ratios on SIF's for crack angle $\theta=2 \pi / 9$. Similar trends as stated by Sarikaya are found. As $E_{2} / E_{1}$ ratio decreases, mode I SIF's increase while mode II SIF's decrease. On the other hand, $E_{3} / E_{1}$ ratio does not lead to critical changes for both mode I and mode II SIF's.

Figure 3.19-Figure 3.20, Figure 3.30-Figure 3.31 and Figure 3.41-Figure 3.42 present the results about SIF's versus crack angle. In these figures, SIF's fluctuate with the change in crack angle.

Related figures concerning the parameters mentioned above are also given for closed cracks loaded by flat punch indentation in Figure 3.43-Figure 3.49. In those figures, both inclined and vertical edge cracks are examined. The direction of friction between crack faces is taken as negative due to forward slipping of crack faces as shown in Figure 3.49. That's why a small drop is observed in mode II SIF's for all cases. To avoid sticking, analyses are performed with low values of $\eta_{2}$. It is observed that full length closure mode occurs only for straight cracks when $\eta_{1}=0$, while crack tip closure is detected in all other cases. In Figure 3.46b, contact pressure distributions between crack faces are presented for an inclined crack $(\theta=\pi / 12)$. In this figure, contact stress at $X=e_{1}$ is found as zero as expected since mode I stress intensity factor is zero at that point. Another set of results in Figure 3.47 and Figure 3.48 are also given to show the influence of other parameters in the problem on contact pressure distribution between crack faces. 


\subsection{Figures for Orthotropic Half-Plane Indented by Flat Stamp (Fully Open}

\section{Crack Assumption)}
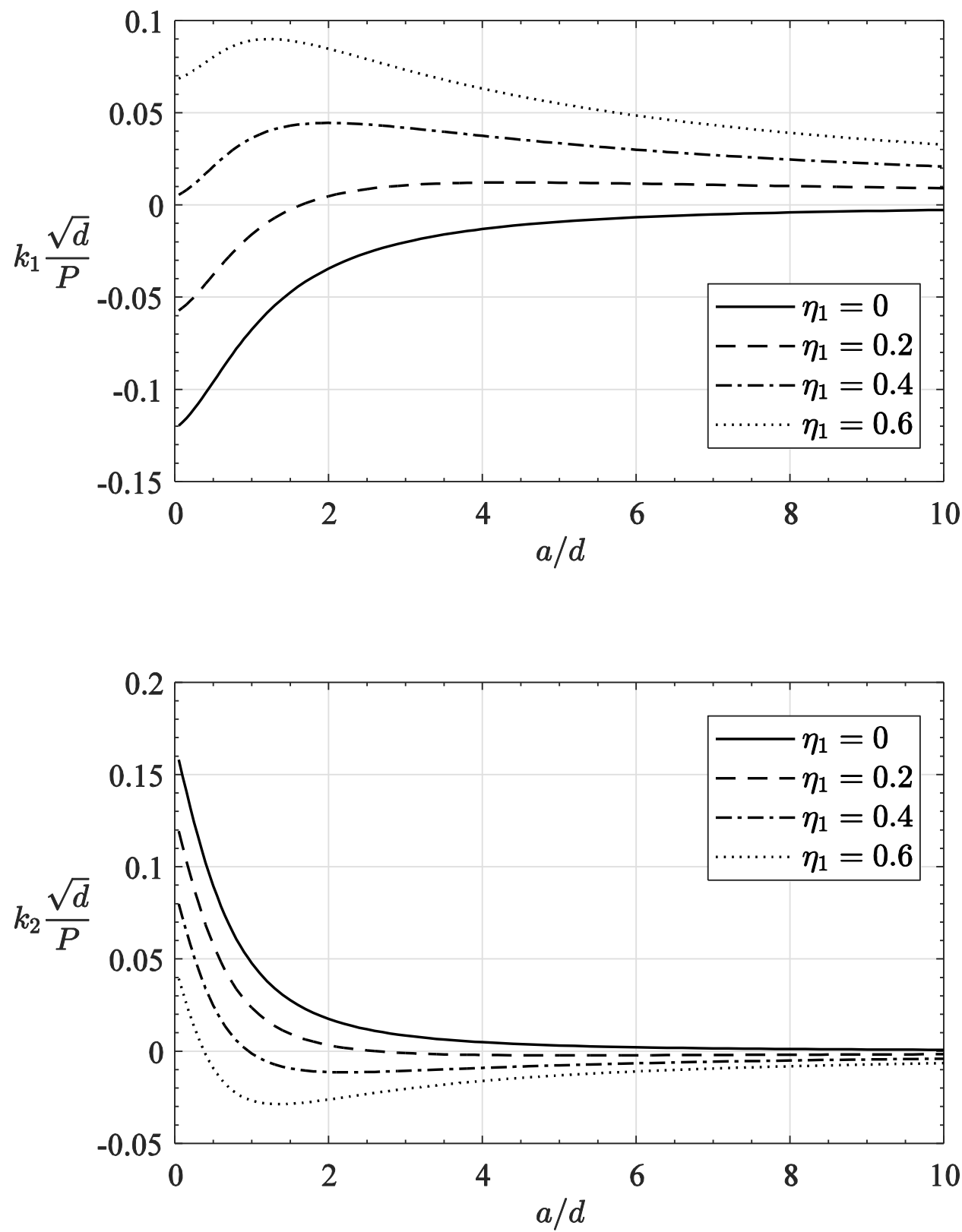

Figure 3.10 Mixed Mode Stress Intensity Factors for Inclined Edge Crack in Orthotropic Half-Plane of Plasma Sprayed Alumina Loaded by Flat Punch for Plane Strain Case and Fully Open Crack Assumption, $(b-a) / d=1$, Crack Angle $\theta=\pi / 12$. 

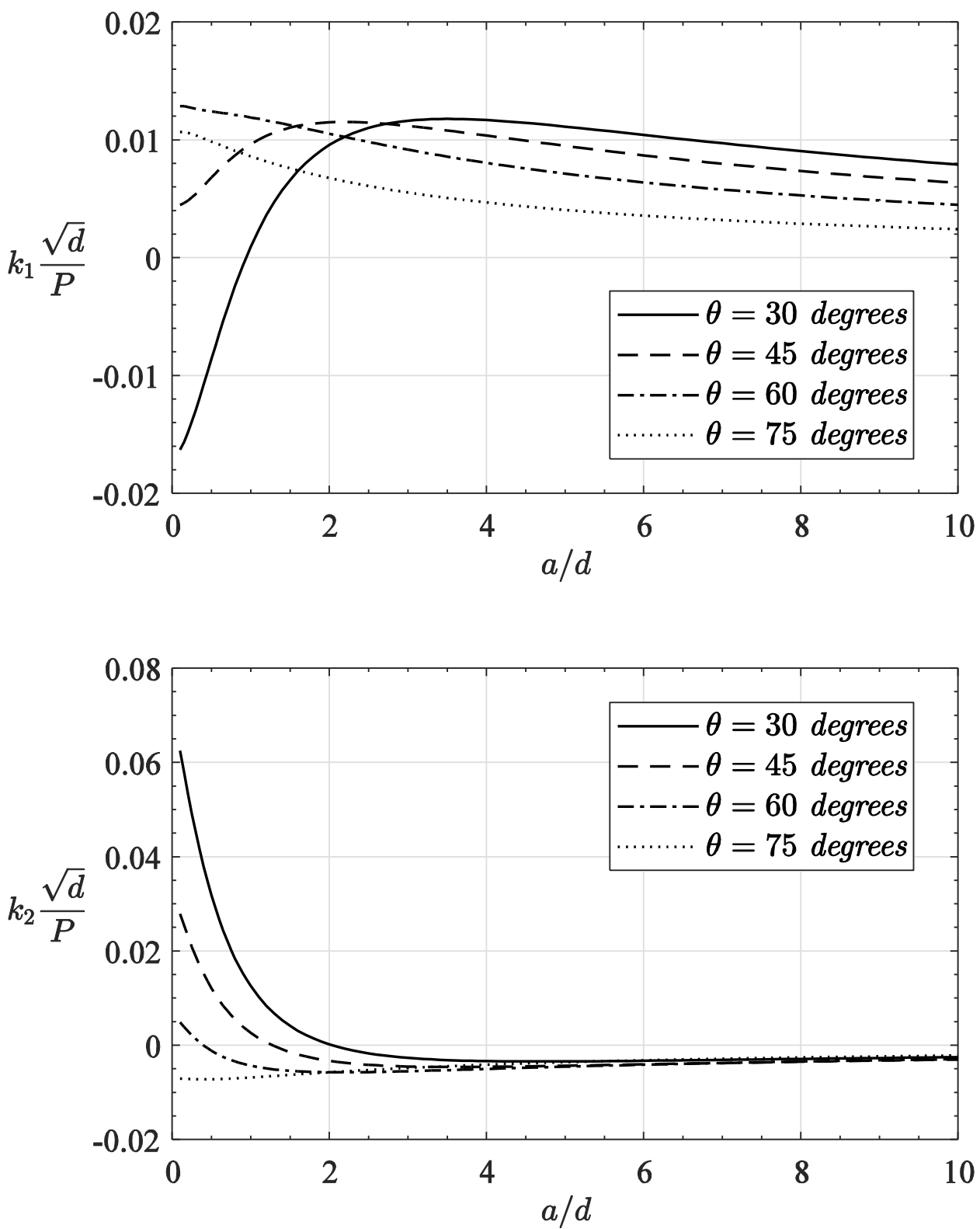

Figure 3.11 Mixed Mode Stress Intensity Factors for Inclined Edge Crack in Orthotropic Half-Plane of Plasma Sprayed Alumina Loaded by Flat Punch for Plane Strain Case and Fully Open Crack Assumption, $(b-a) / d=1, \eta_{1}=0.2$. 

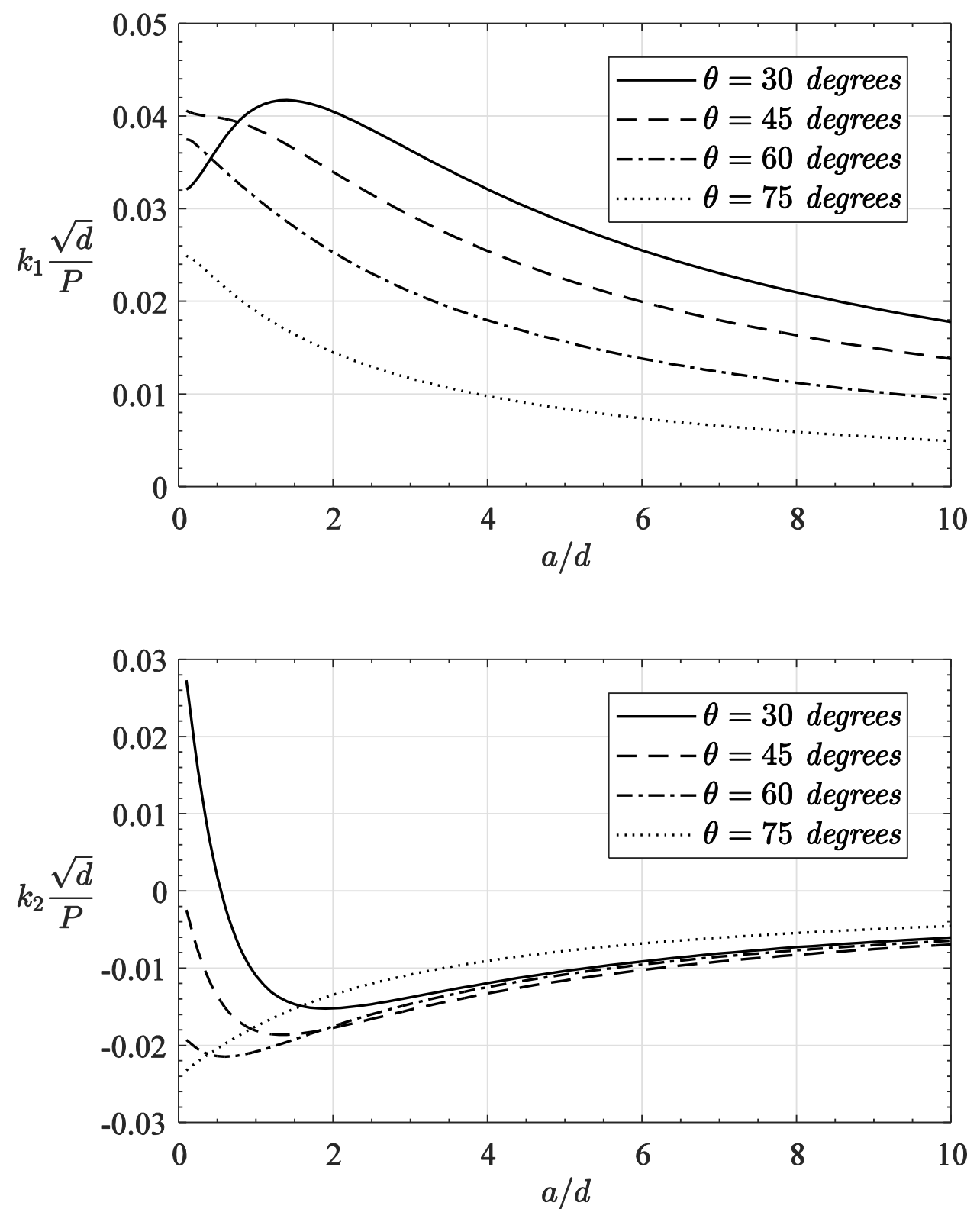

Figure 3.12 Mixed Mode Stress Intensity Factors for Inclined Edge Crack in Orthotropic Half-Plane of Plasma Sprayed Alumina Loaded by Flat Punch for Plane Strain Case and Fully Open Crack Assumption, $(b-a) / d=1, \eta_{1}=0.4$. 

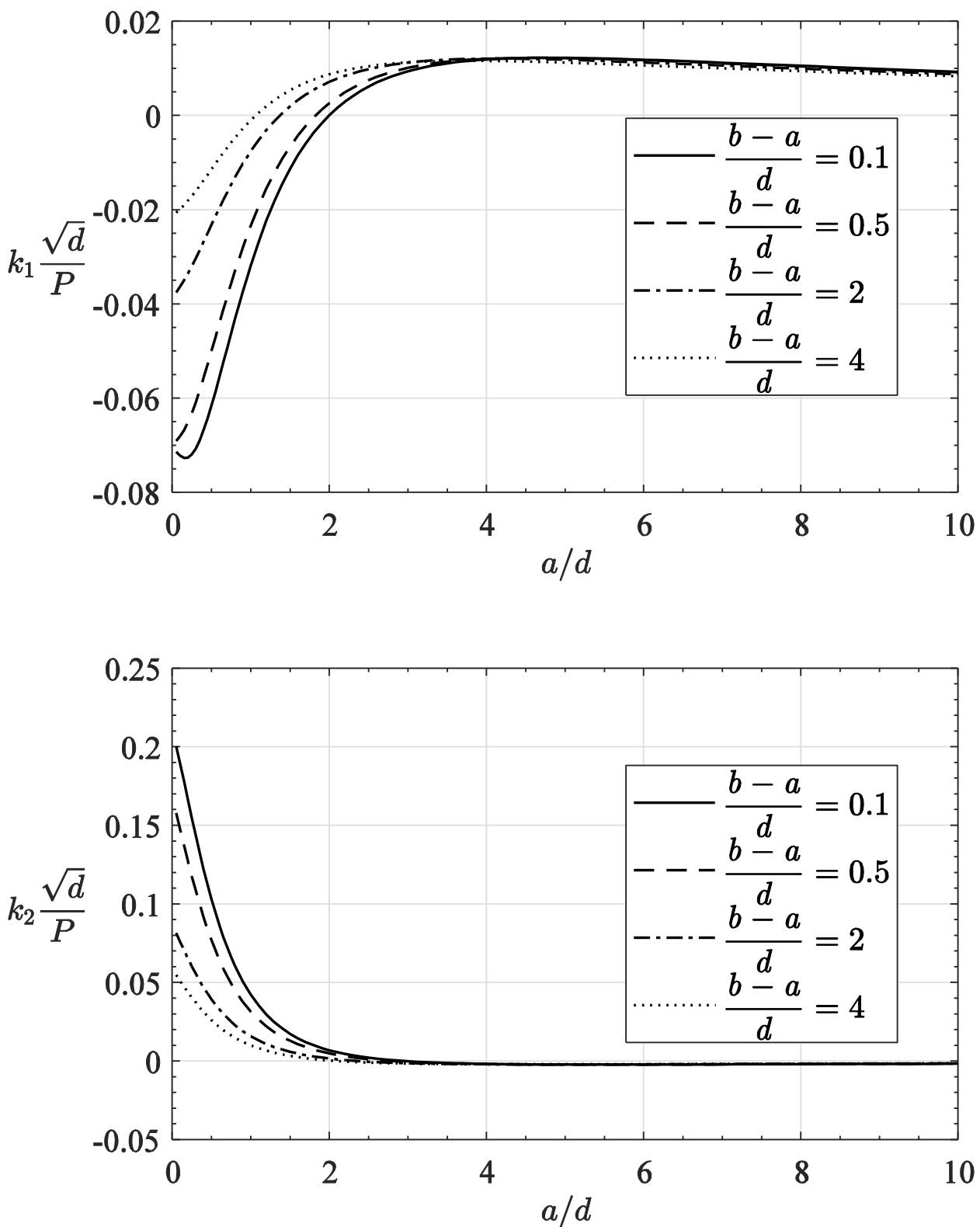

Figure 3.13 Effect of $(b-a) / d$ Variation on Mixed Mode Stress Intensity Factors for Inclined Edge Crack in Orthotropic Half-Plane of Plasma Sprayed Alumina Loaded by Flat Punch for Plane Strain Case and Fully Open Crack Assumption, Crack Angle $\theta=\pi / 12, \eta_{1}=0.2$. 

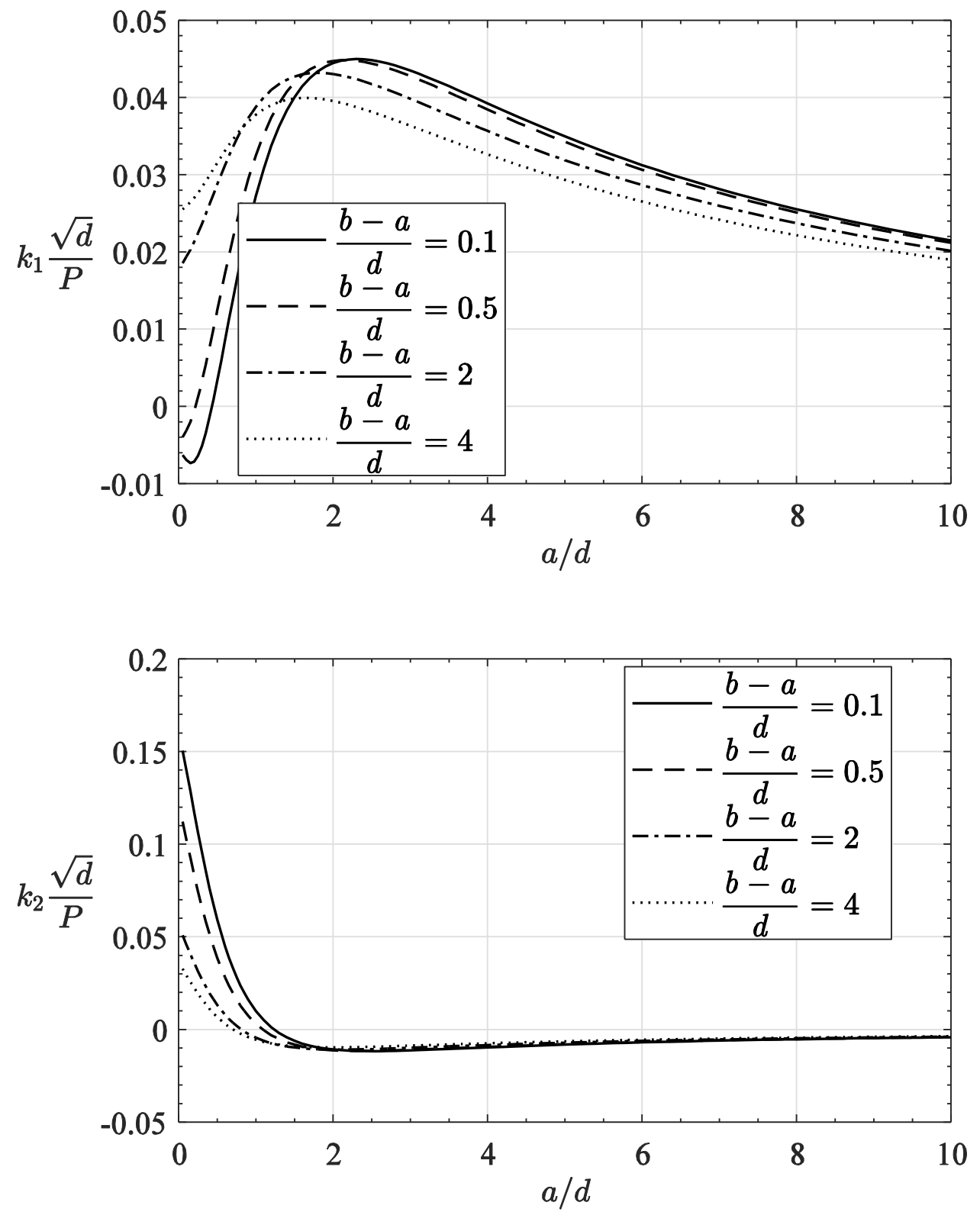

Figure 3.14 Effect of $(b-a) / d$ Variation on Mixed Mode Stress Intensity Factors for Inclined Edge Crack in Orthotropic Half-Plane of Plasma Sprayed Alumina Loaded by Flat Punch for Plane Strain Case and Fully Open Crack Assumption, Crack Angle $\theta=\pi / 12, \eta_{1}=0.4$. 

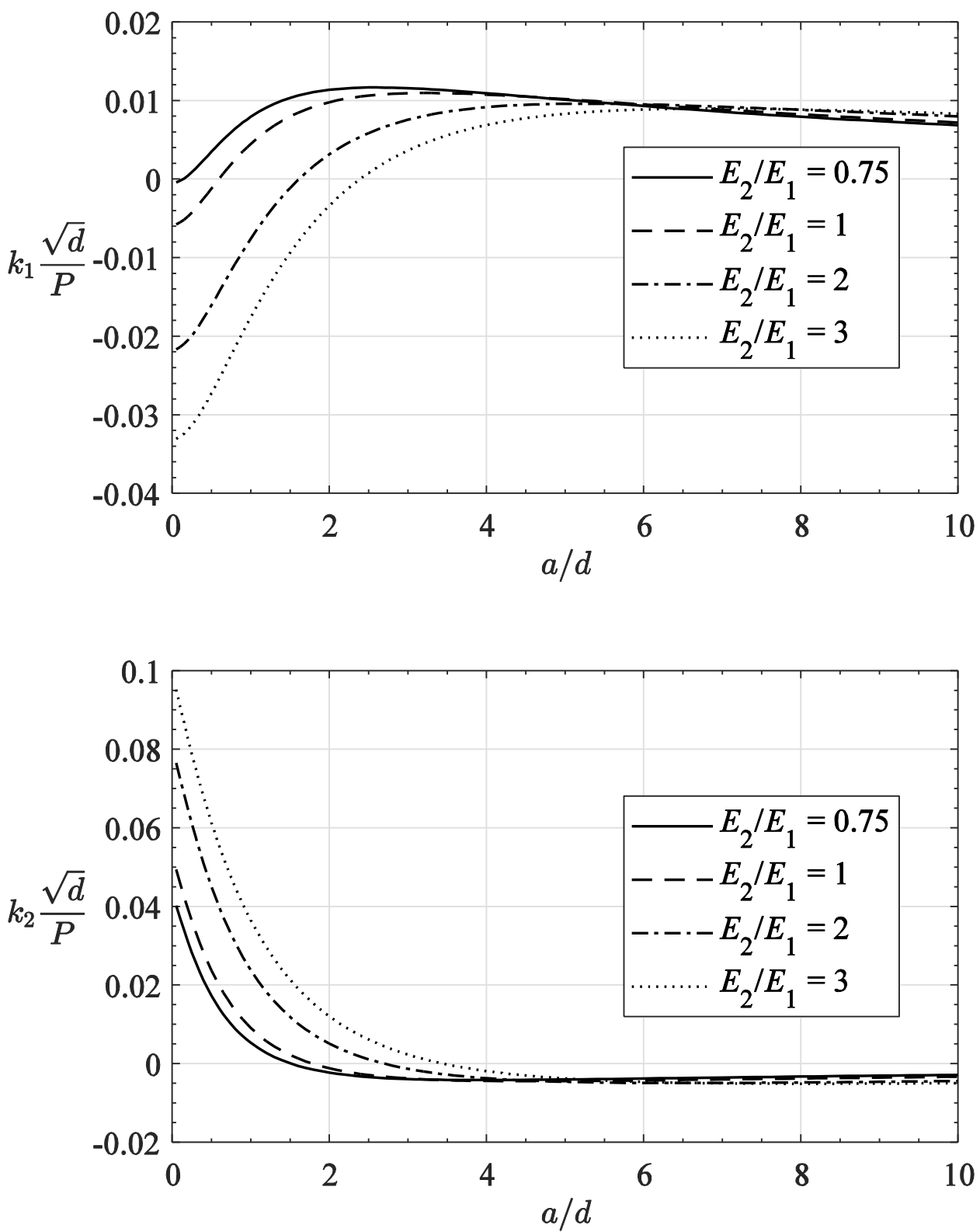

Figure 3.15 Effect of Elastic Modulus Ratio $E_{2} / E_{1}$ on Mixed Mode Stress Intensity Factors for Inclined Edge Crack in Orthotropic Half-Plane Loaded by Flat Punch for Plane Strain Case and Fully Open Crack Assumption, $(b-a) / d=$ 1, Crack Angle $\theta=2 \pi / 9, \eta_{1}=0.2$. 

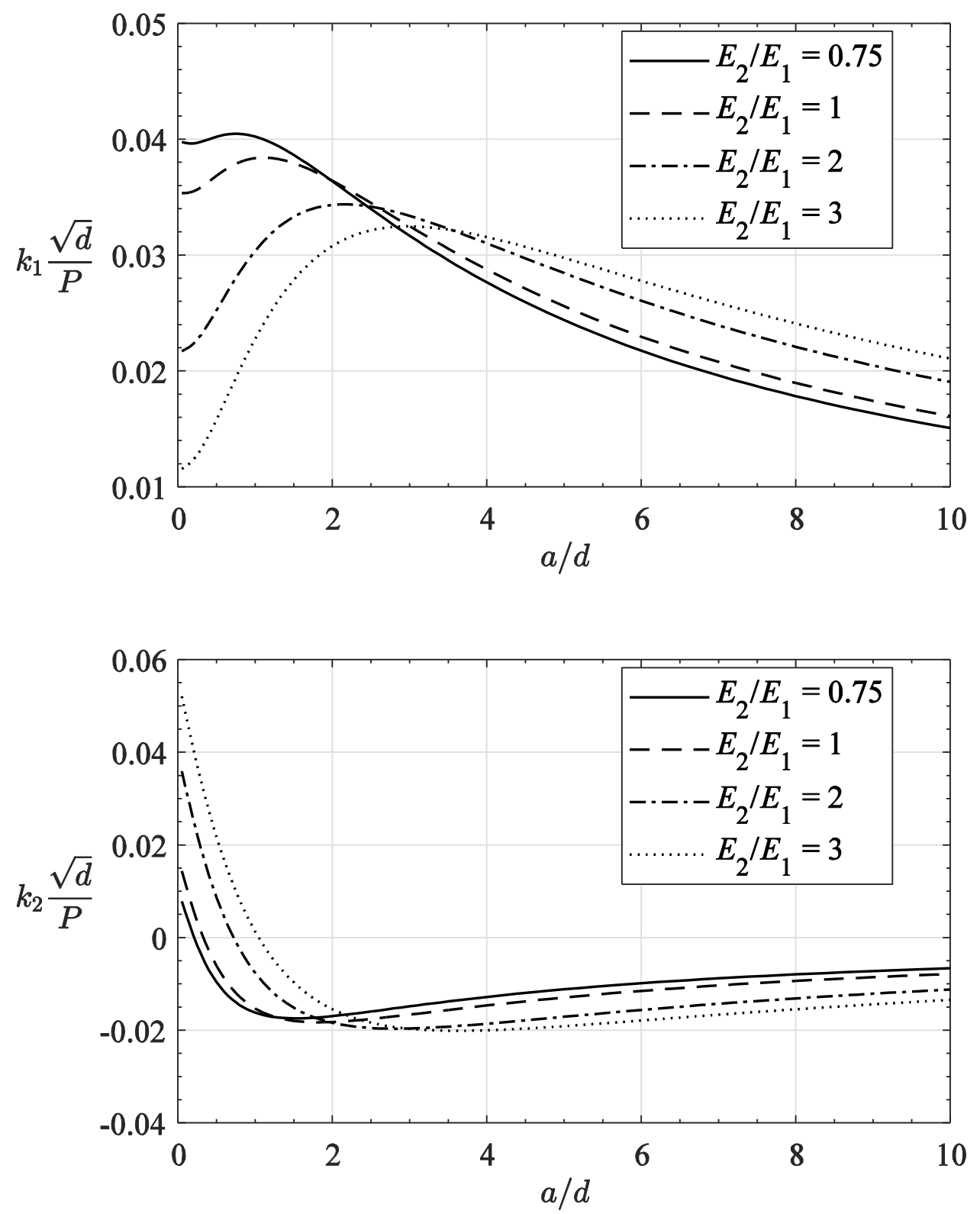

Figure 3.16 Effect of Elastic Modulus Ratio $E_{2} / E_{1}$ on Mixed Mode Stress Intensity Factors for Inclined Edge Crack in Orthotropic Half-Plane Loaded by Flat Punch for Plane Strain Case and Fully Open Crack Assumption, $(b-a) / d=$ 1 , Crack Angle $\theta=2 \pi / 9, \eta_{1}=0.4$. 

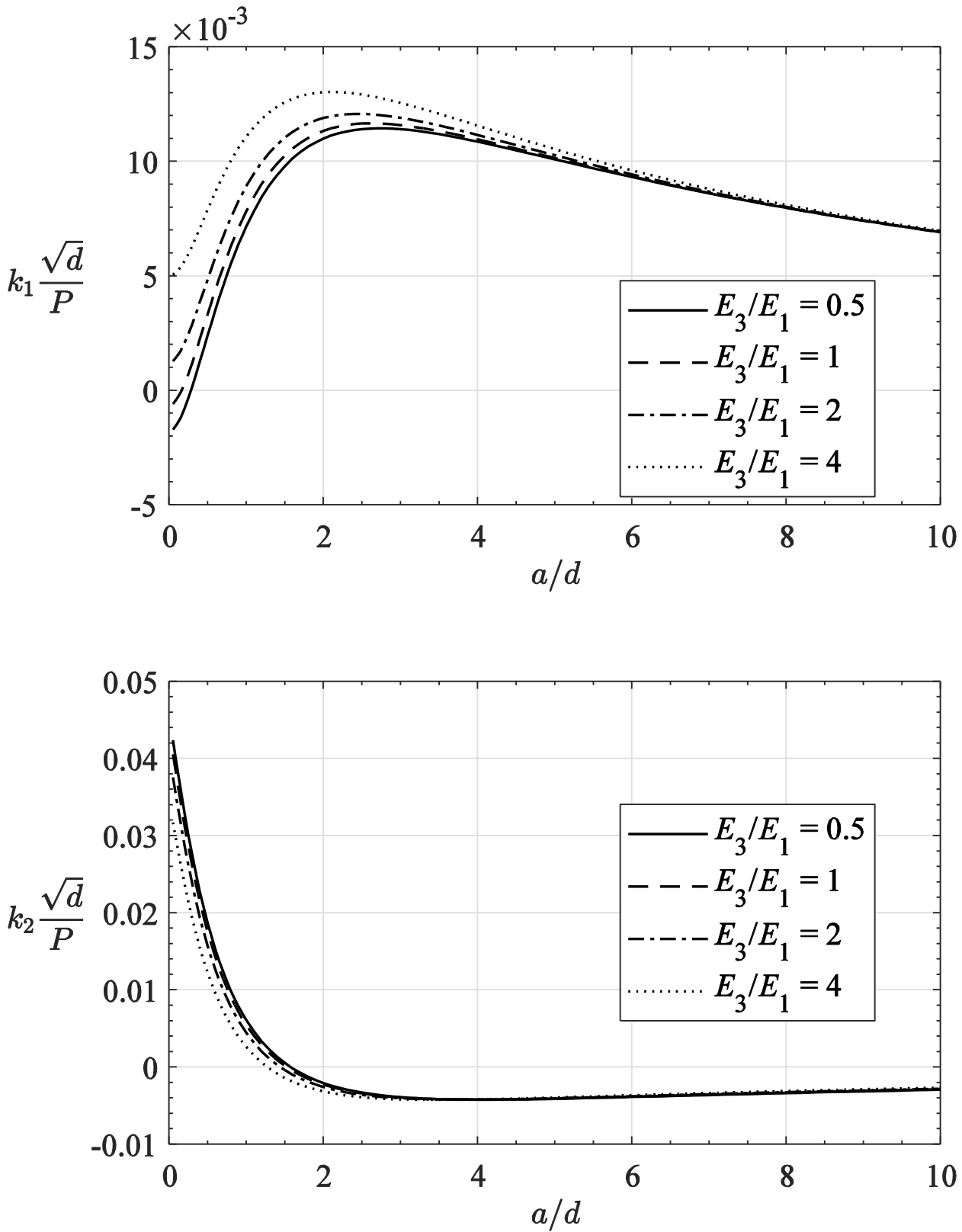

Figure 3.17 Effect of Elastic Modulus Ratio $E_{3} / E_{1}$ on Mixed Mode Stress Intensity Factors for Inclined Edge Crack in Orthotropic Half-Plane Loaded by Flat Punch for Plane Strain Case and Fully Open Crack Assumption, $(b-a) / d=$ 1 , Crack Angle $\theta=2 \pi / 9, \eta_{1}=0.2$. 

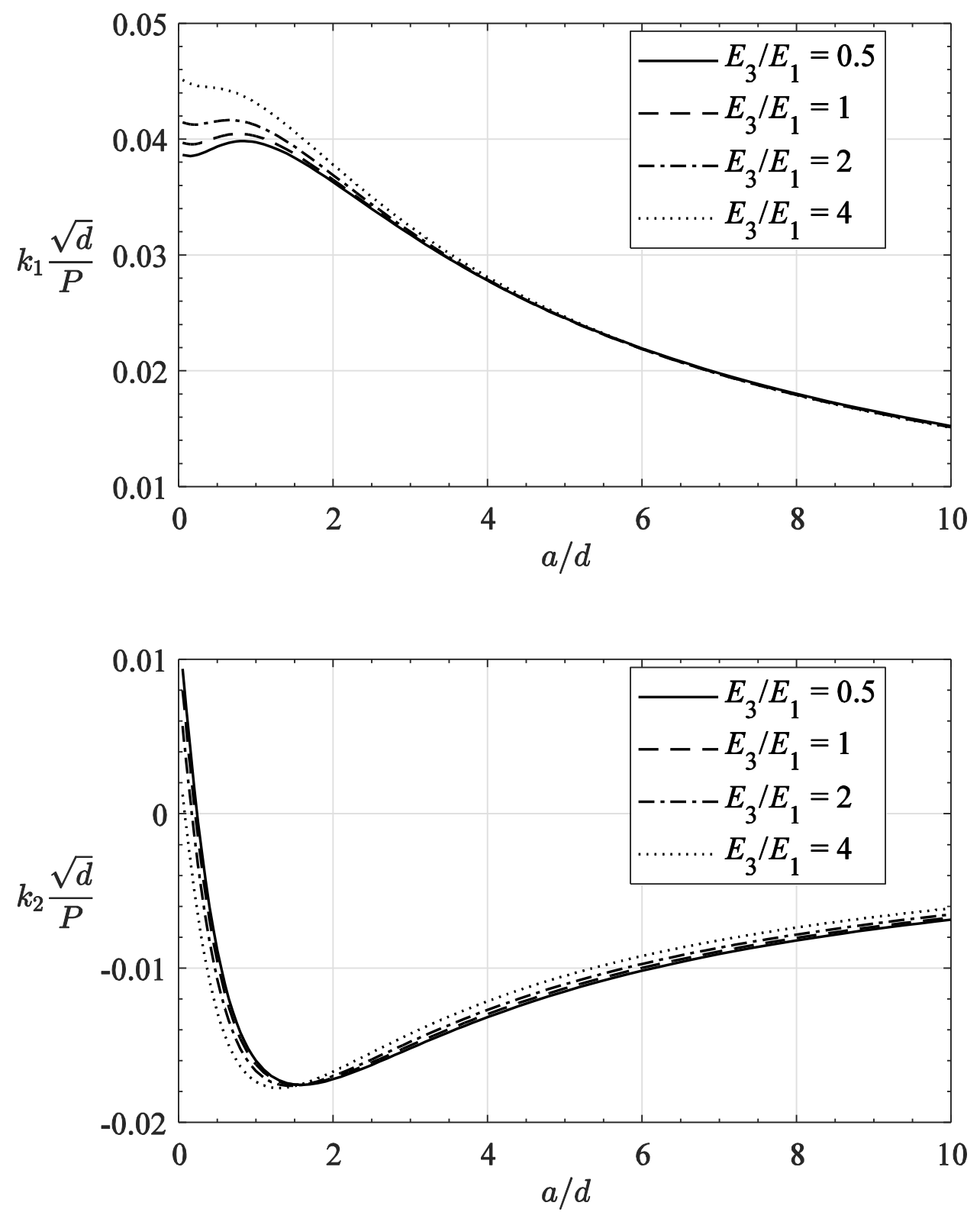

Figure 3.18 Effect of Elastic Modulus Ratio $E_{3} / E_{1}$ on Mixed Mode Stress Intensity Factors for Inclined Edge Crack in Orthotropic Half-Plane Loaded by Flat Punch for Plane Strain Case and Fully Open Crack Assumption, $(b-a) / d=$ 1, Crack Angle $\theta=2 \pi / 9, \eta_{1}=0.4$. 

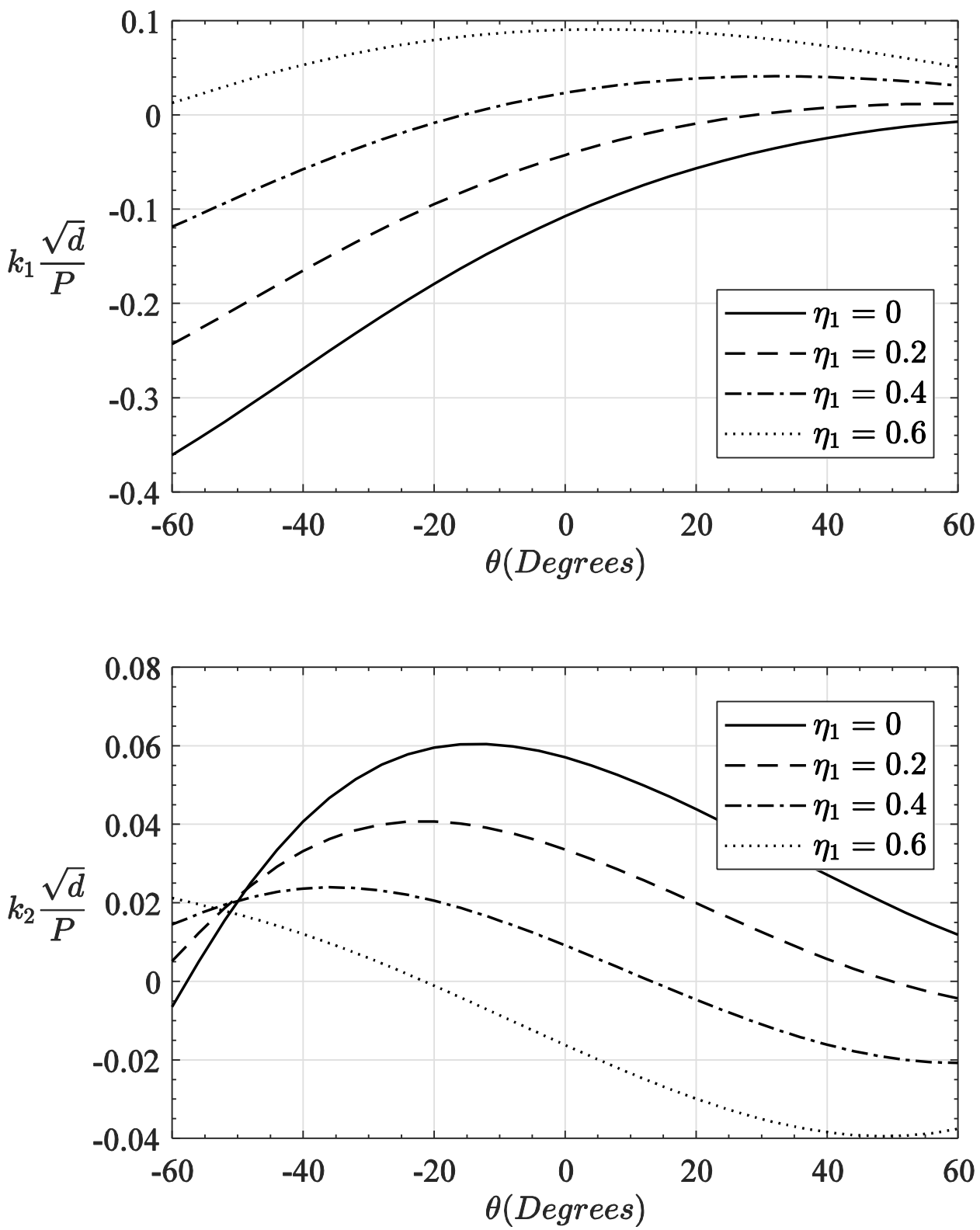

Figure 3.19 Effect of Crack Angle Variation on Mixed Mode Stress Intensity Factors for Inclined Edge Crack in Orthotropic Half-Plane of Plasma Sprayed Alumina Loaded by Flat Punch for Plane Strain Case and Fully Open Crack Assumption, $(b-a) / d=1, a / d=1$. 

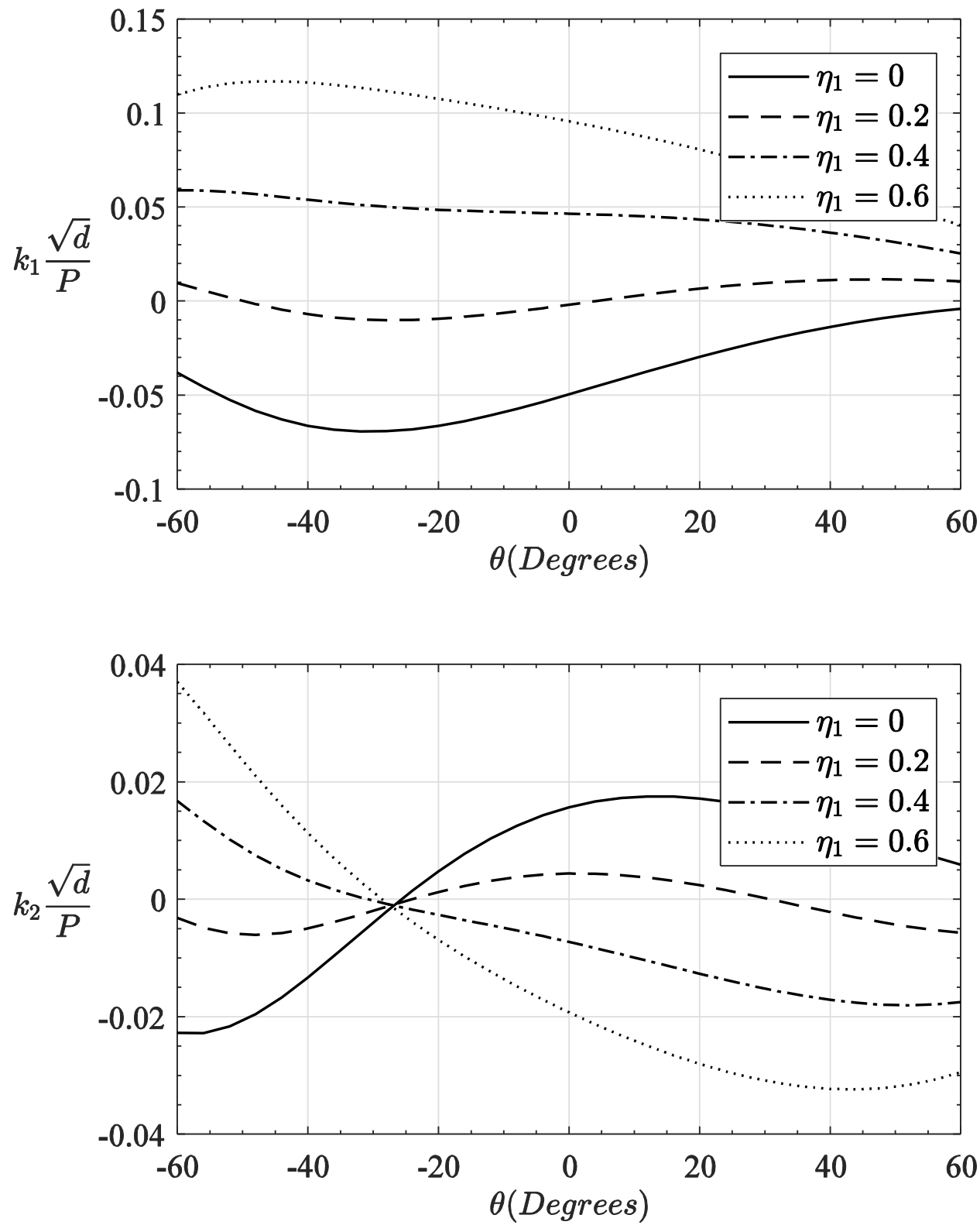

Figure 3.20 Effect of Crack Angle Variation on Mixed Mode Stress Intensity Factors for Inclined Edge Crack in Orthotropic Half-Plane of Plasma Sprayed Alumina Loaded by Flat Punch for Plane Strain Case and Fully Open Crack Assumption, $(b-a) / d=1, a / d=2$. 
3.2 Figures for Orthotropic Half-Plane Indented by Triangular Stamp (Fully Open Crack Assumption)
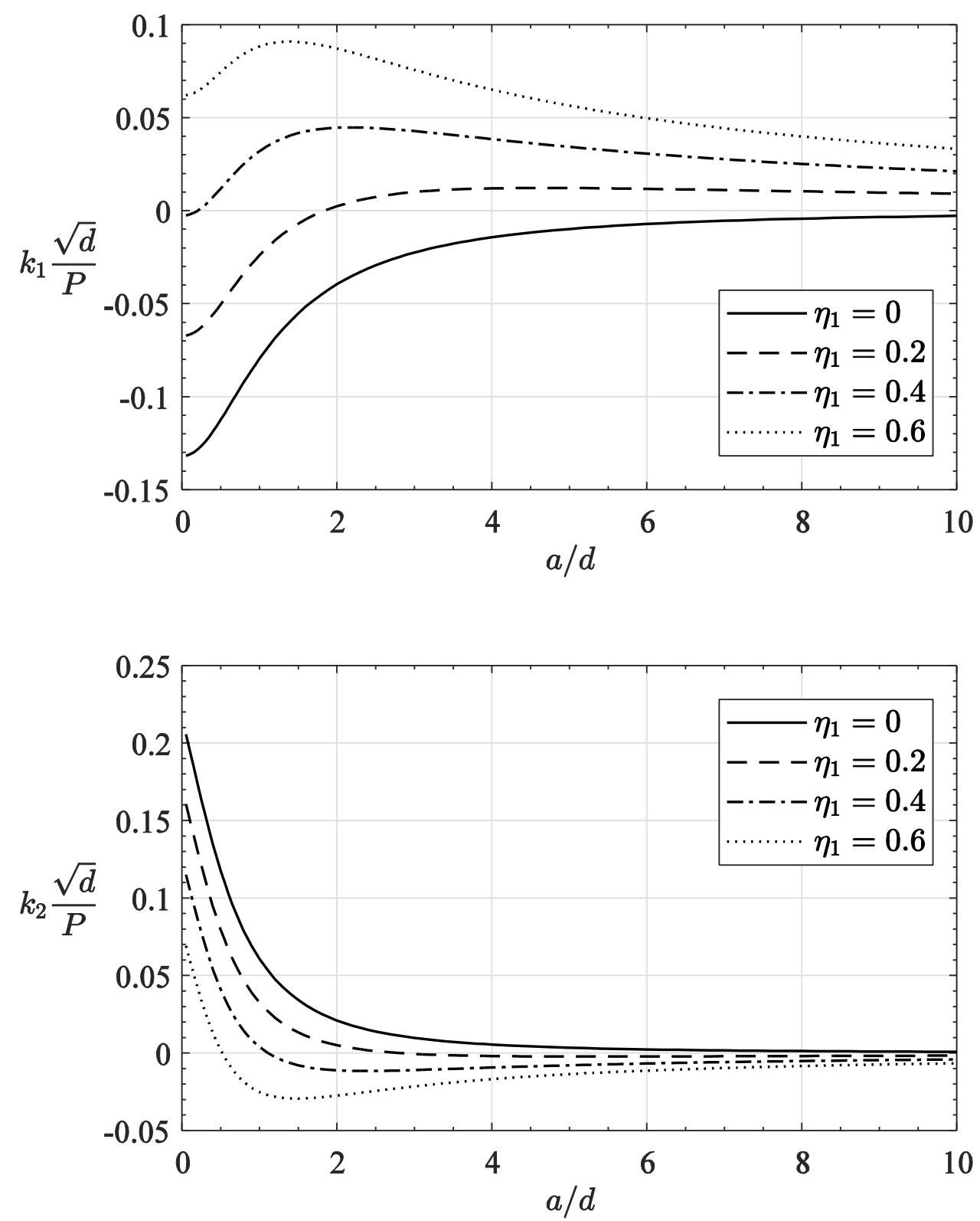

Figure 3.21 Mixed Mode Stress Intensity Factors for Inclined Edge Crack in Orthotropic Half-Plane of Plasma Sprayed Alumina Loaded by Triangular Punch for Plane Strain Case and Fully Open Crack Assumption, $(b-a) / d=1$, Crack Angle $\theta=\pi / 12$. 

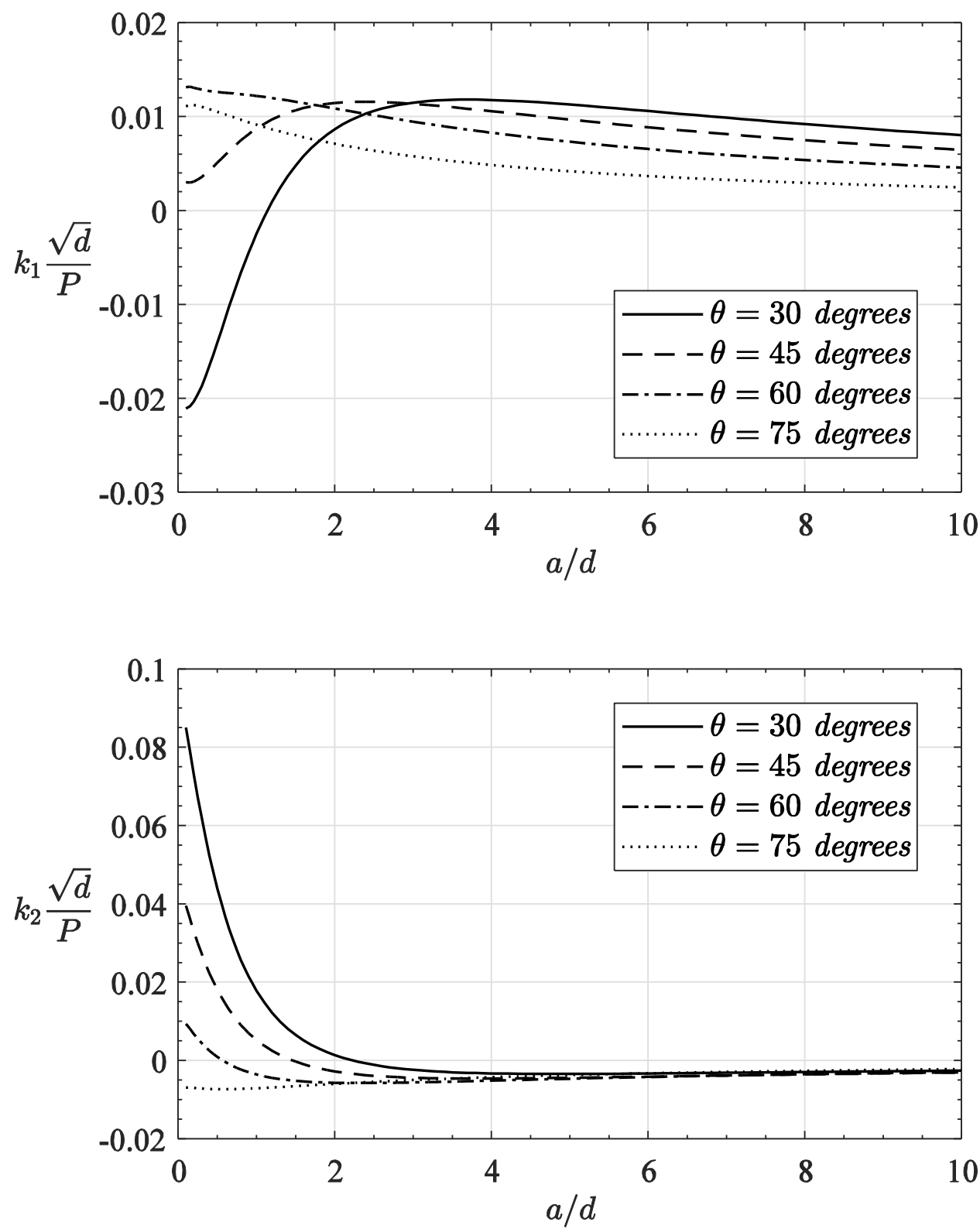

Figure 3.22 Mixed Mode Stress Intensity Factors for Inclined Edge Crack in Orthotropic Half-Plane of Plasma Sprayed Alumina Loaded by Triangular Punch for Plane Strain Case and Fully Open Crack Assumption, $(b-a) / d=1, \eta_{1}$ $=0.2$. 

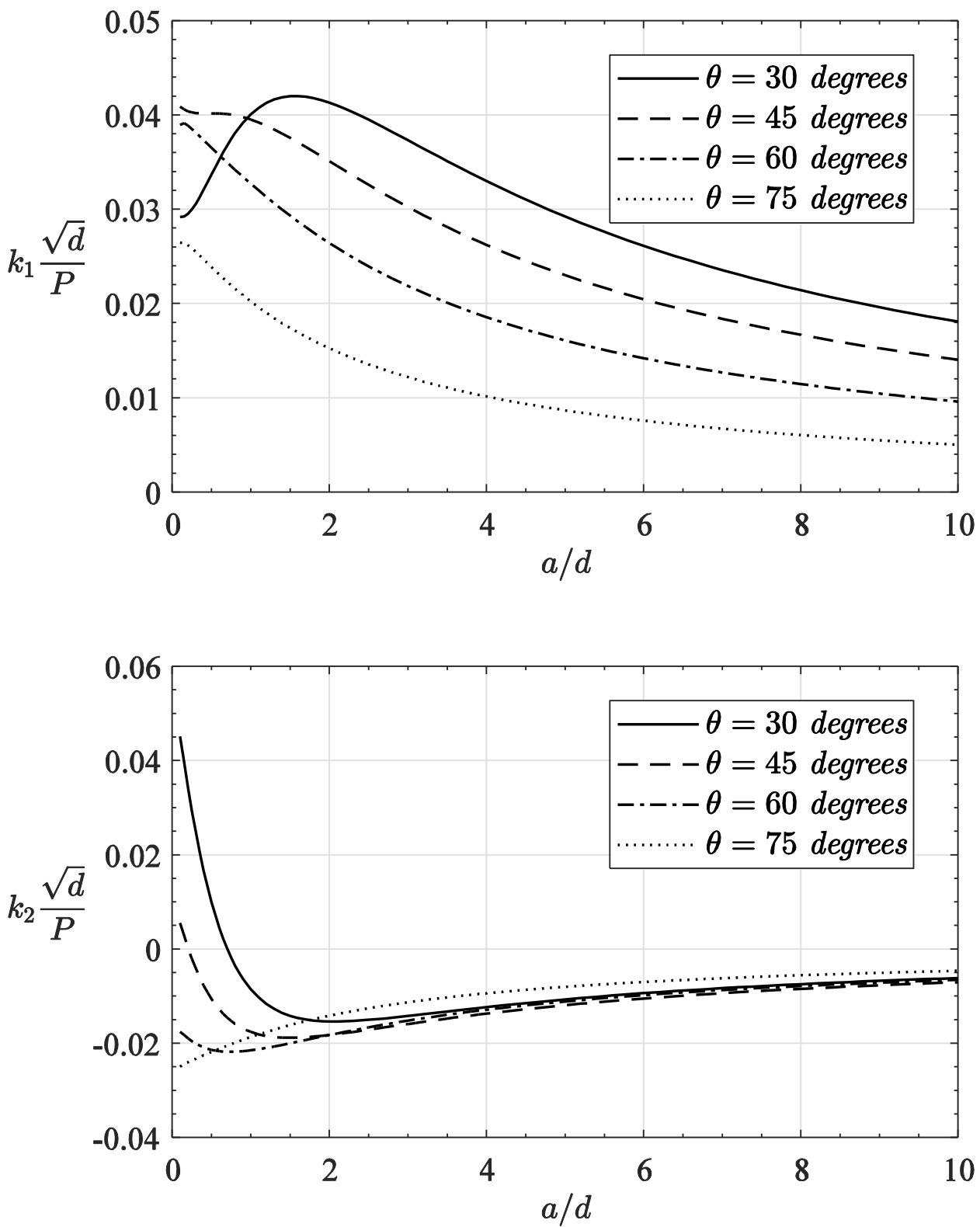

Figure 3.23 Mixed Mode Stress Intensity Factors for Inclined Edge Crack in Orthotropic Half-Plane of Plasma Sprayed Alumina Loaded by Triangular Punch for Plane Strain Case and Fully Open Crack Assumption, $(b-a) / d=1, \eta_{1}$ $=0.4$. 

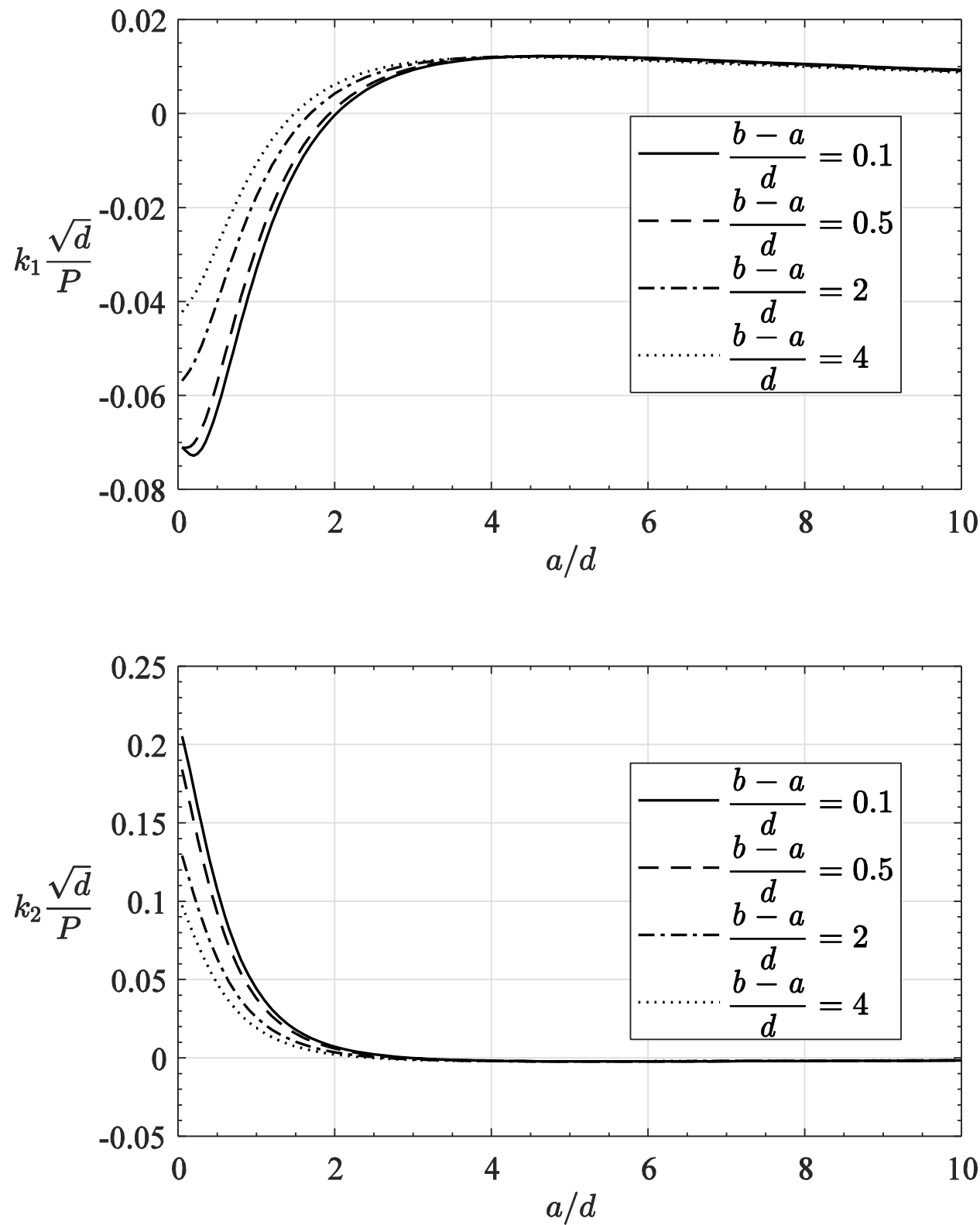

Figure 3.24 Effect of $(b-a) / d$ Variation on Mixed Mode Stress Intensity Factors for Inclined Edge Crack in Orthotropic Half-Plane of Plasma Sprayed Alumina Loaded by Triangular Punch for Plane Strain Case and Fully Open Crack Assumption, Crack Angle $\theta=\pi / 12, \eta_{1}=0.2$. 

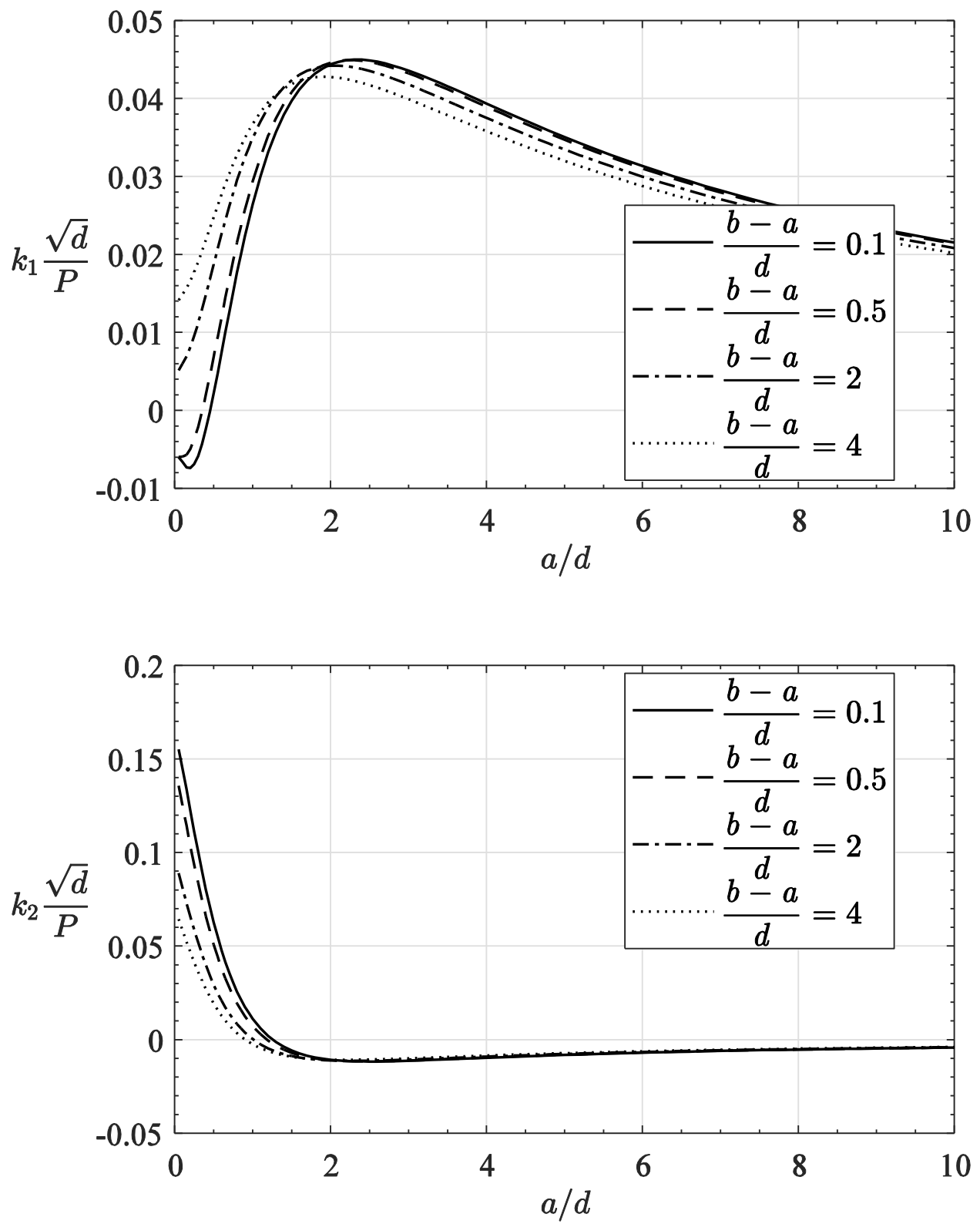

Figure 3.25 Effect of $(b-a) / d$ Variation on Mixed Mode Stress Intensity Factors for Inclined Edge Crack in Orthotropic Half-Plane of Plasma Sprayed Alumina Loaded by Triangular Punch for Plane Strain Case and Fully Open Crack Assumption, Crack Angle $\theta=\pi / 12, \eta_{1}=0.4$. 

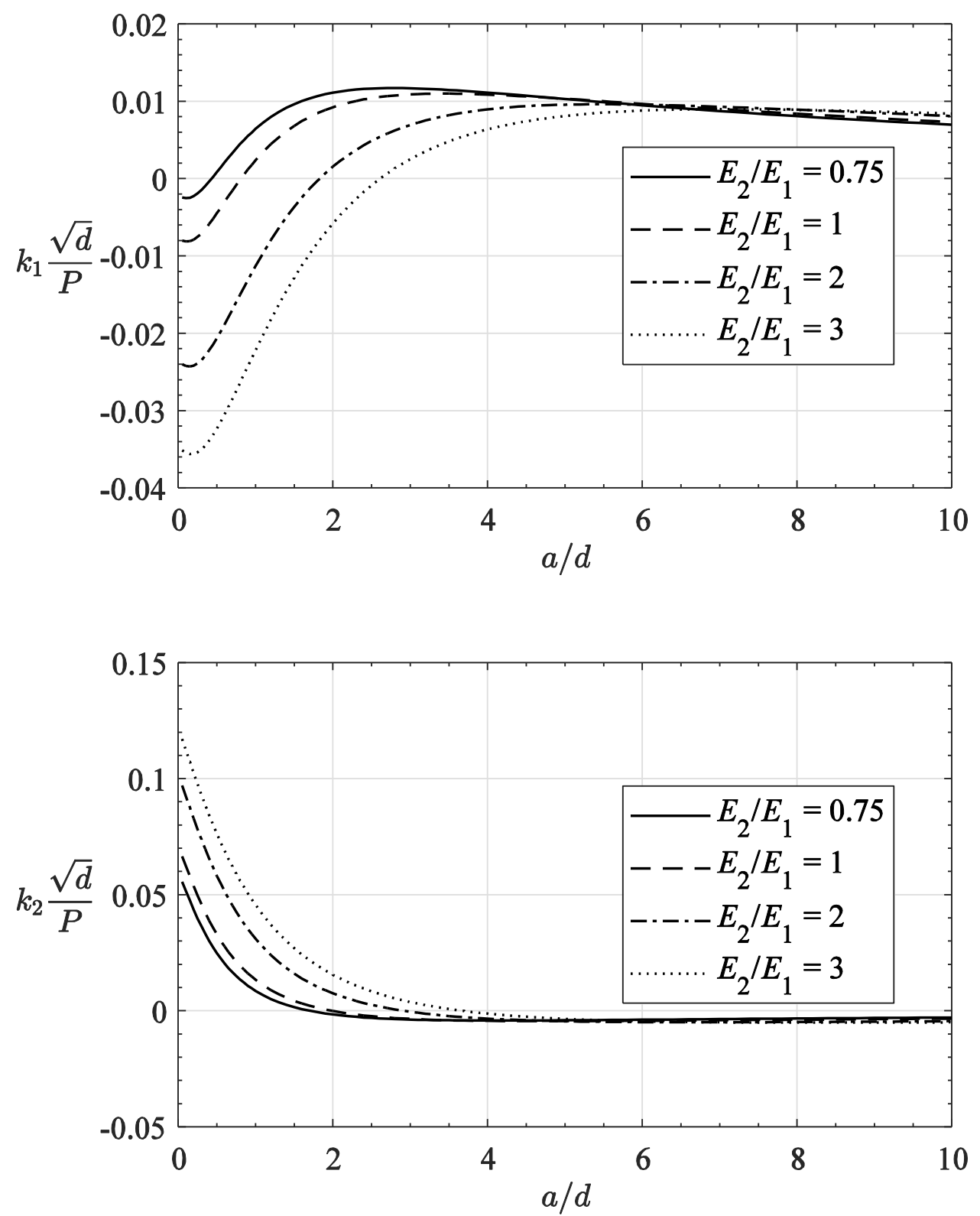

Figure 3.26 Effect of Elastic Modulus Ratio $E_{2} / E_{1}$ on Mixed Mode Stress Intensity Factors for Inclined Edge Crack in Orthotropic Half-Plane Loaded by Triangular Punch for Plane Strain Case and Fully Open Crack Assumption, $(b-a) / d=1$, Crack Angle $\theta=2 \pi / 9, \eta_{1}=0.2$. 

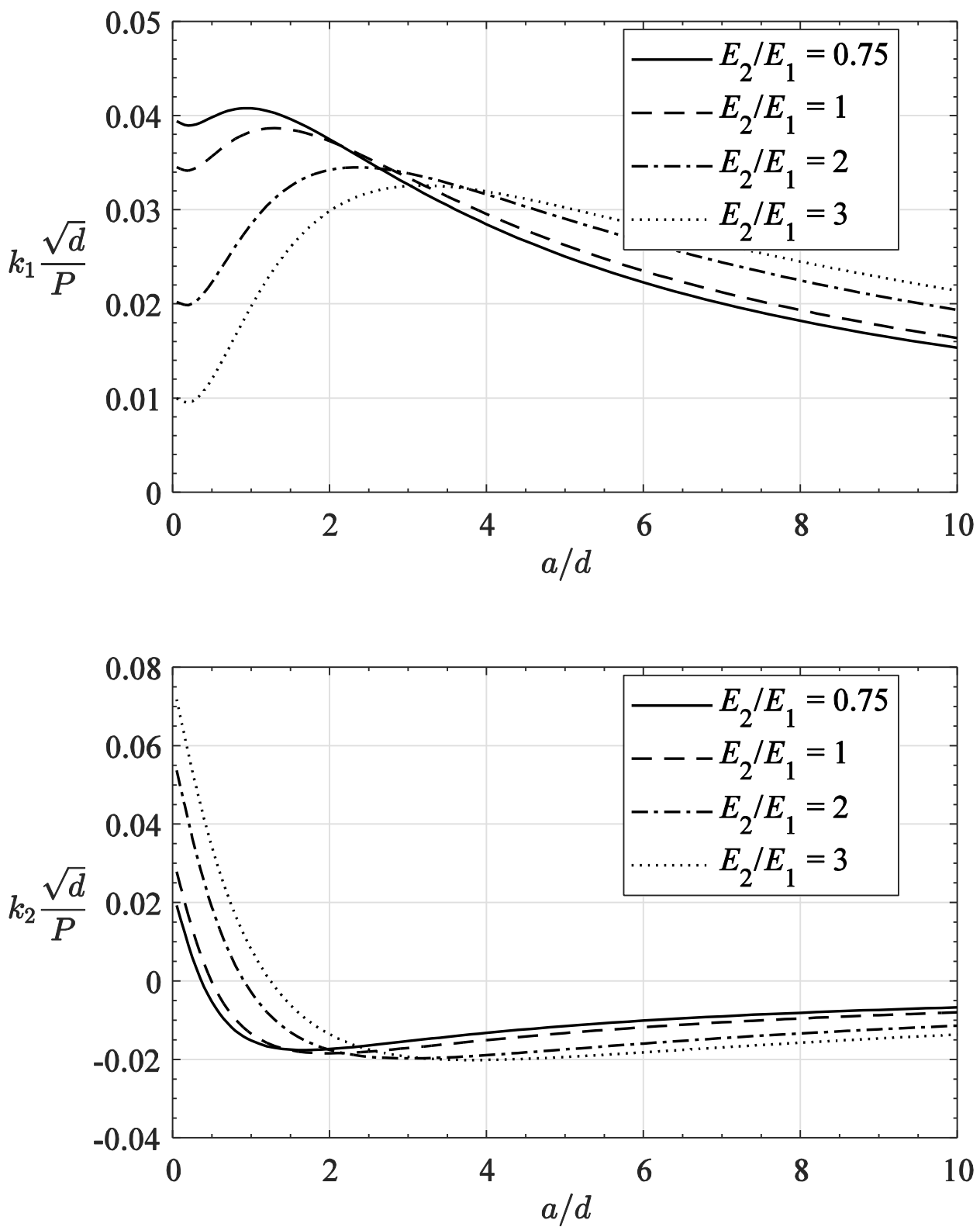

Figure 3.27 Effect of Elastic Modulus Ratio $E_{2} / E_{1}$ on Mixed Mode Stress Intensity Factors for Inclined Edge Crack in Orthotropic Half-Plane Loaded by Triangular Punch for Plane Strain Case and Fully Open Crack Assumption, $(b-a) / d=1$, Crack Angle $\theta=2 \pi / 9, \eta_{1}=0.4$. 

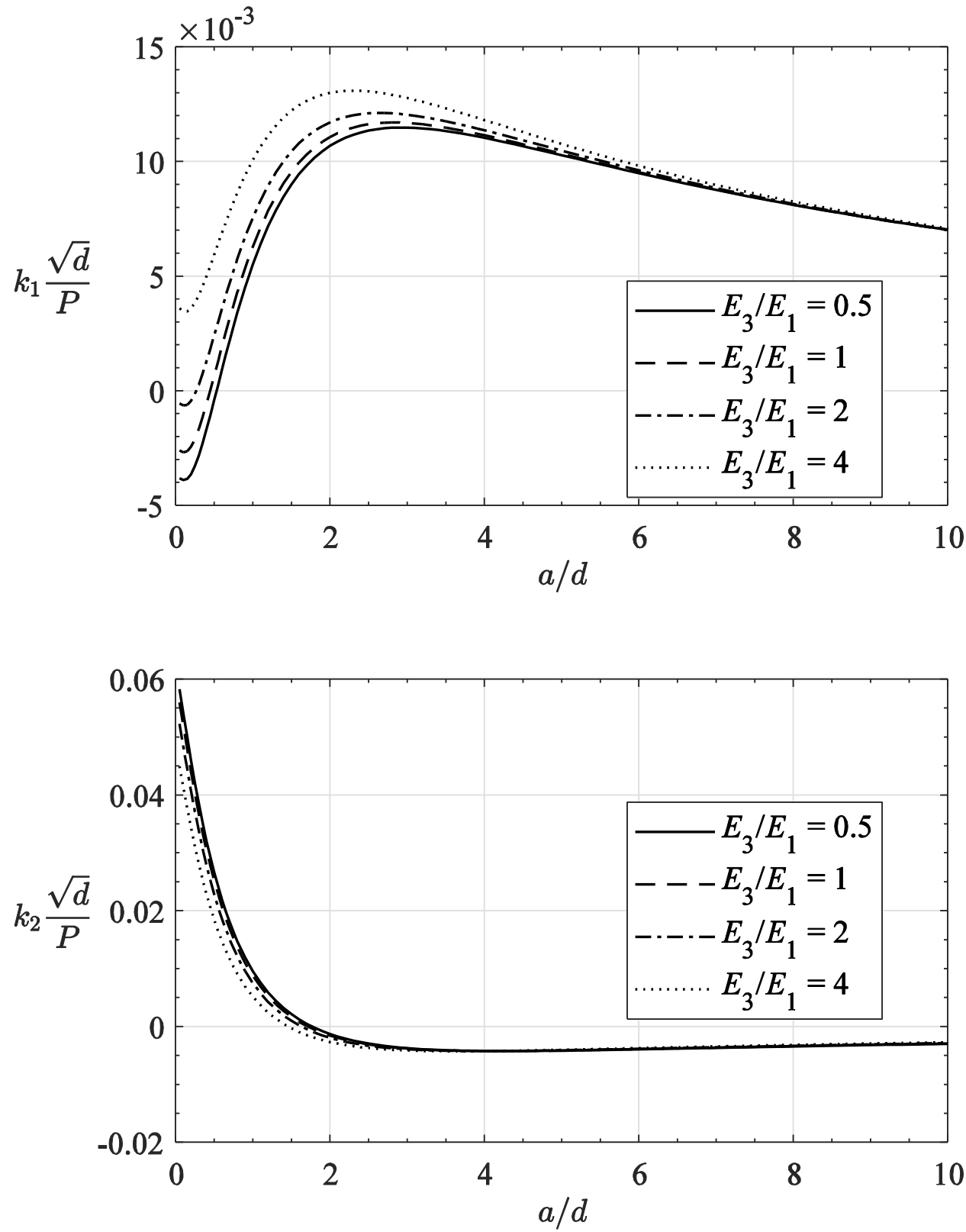

Figure 3.28 Effect of Elastic Modulus Ratio $E_{3} / E_{1}$ on Mixed Mode Stress Intensity Factors for Inclined Edge Crack in Orthotropic Half-Plane Loaded by Triangular Punch for Plane Strain Case and Fully Open Crack Assumption, $(b-a) / d=1$, Crack Angle $\theta=2 \pi / 9, \eta_{1}=0.2$. 

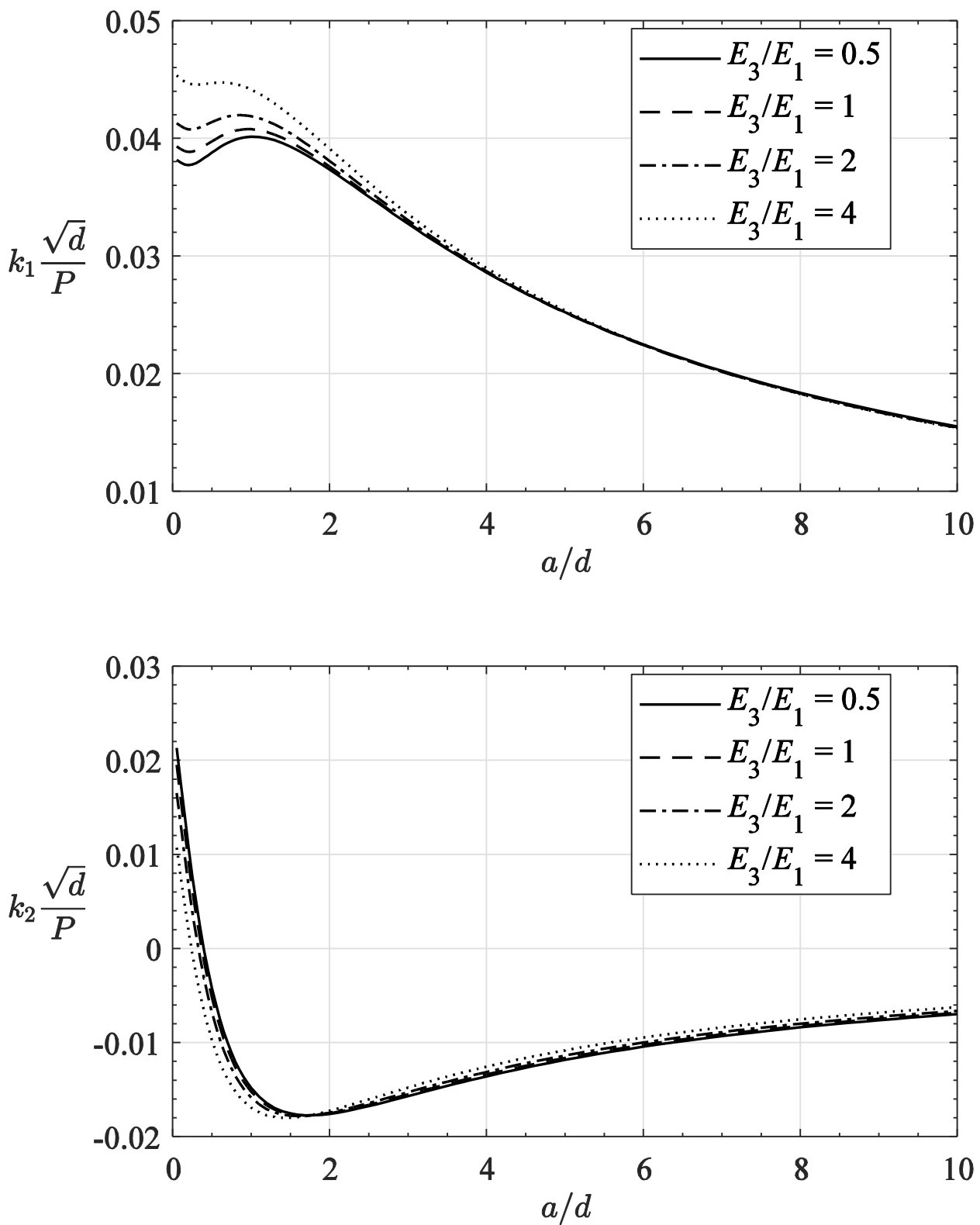

Figure 3.29 Effect of Elastic Modulus Ratio $E_{3} / E_{1}$ on Mixed Mode Stress Intensity Factors for Inclined Edge Crack in Orthotropic Half-Plane Loaded by Triangular Punch for Plane Strain Case and Fully Open Crack Assumption, $(b-a) / d=1$, Crack Angle $\theta=2 \pi / 9, \eta_{1}=0.4$. 

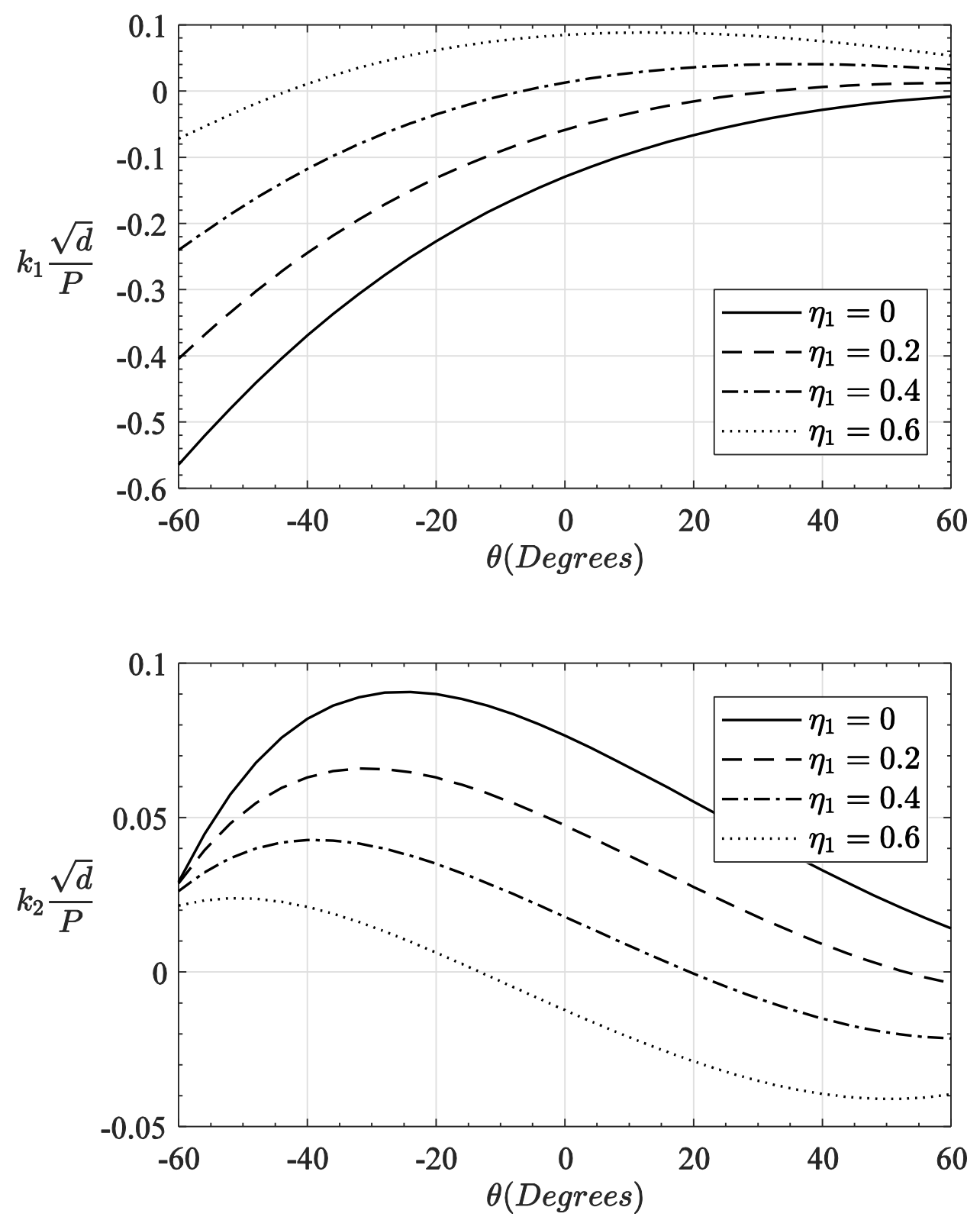

Figure 3.30 Effect of Crack Angle Variation on Mixed Mode Stress Intensity Factors for Inclined Edge Crack in Orthotropic Half-Plane of Plasma Sprayed Alumina Loaded by Triangular Punch for Plane Strain Case and Fully Open Crack Assumption, $(b-a) / d=1, a / d=1$. 

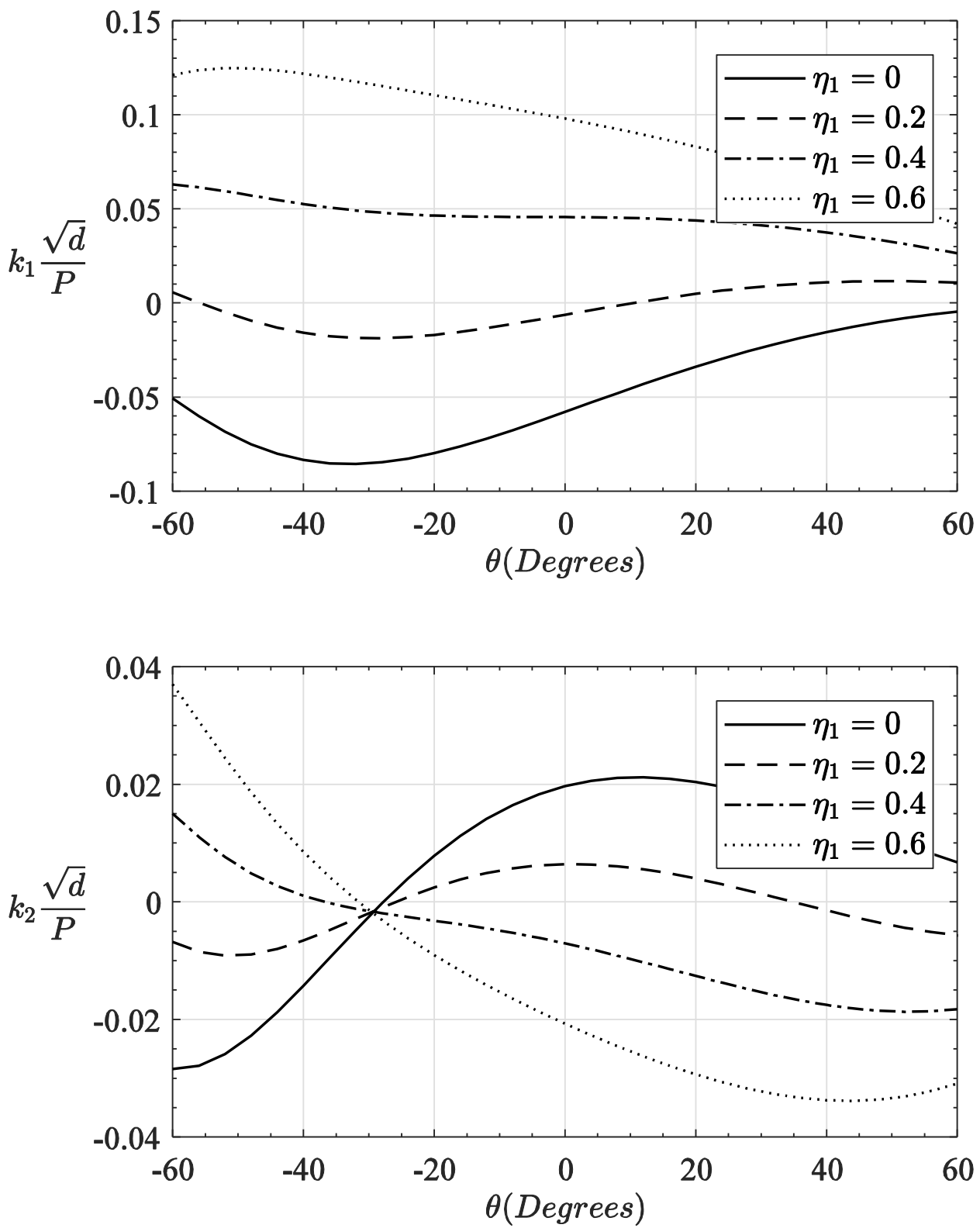

Figure 3.31 Effect of Crack Angle Variation on Mixed Mode Stress Intensity Factors for Inclined Edge Crack in Orthotropic Half-Plane of Plasma Sprayed Alumina Loaded by Triangular Punch for Plane Strain Case and Fully Open Crack Assumption, $(b-a) / d=1, a / d=2$. 
3.3 Figures for Orthotropic Half-Plane Indented by Circular Stamp (Fully Open Crack Assumption)
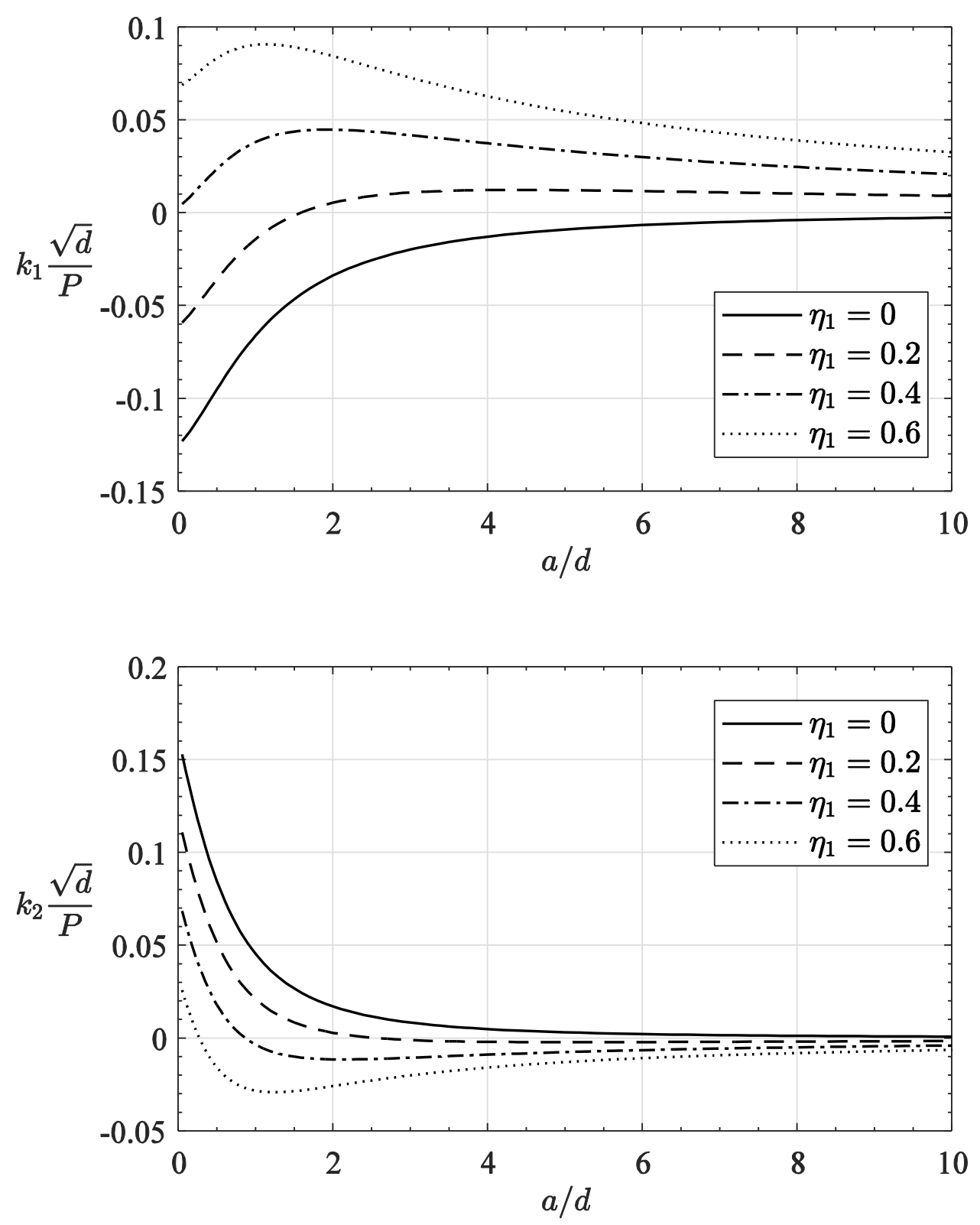

Figure 3.32 Mixed Mode Stress Intensity Factors for Inclined Edge Crack in Orthotropic Half-Plane of Plasma Sprayed Alumina Loaded by Circular Punch for Plane Strain Case and Fully Open Crack Assumption, $(b-a) / d=1$, Crack Angle $\theta=\pi / 12$. 

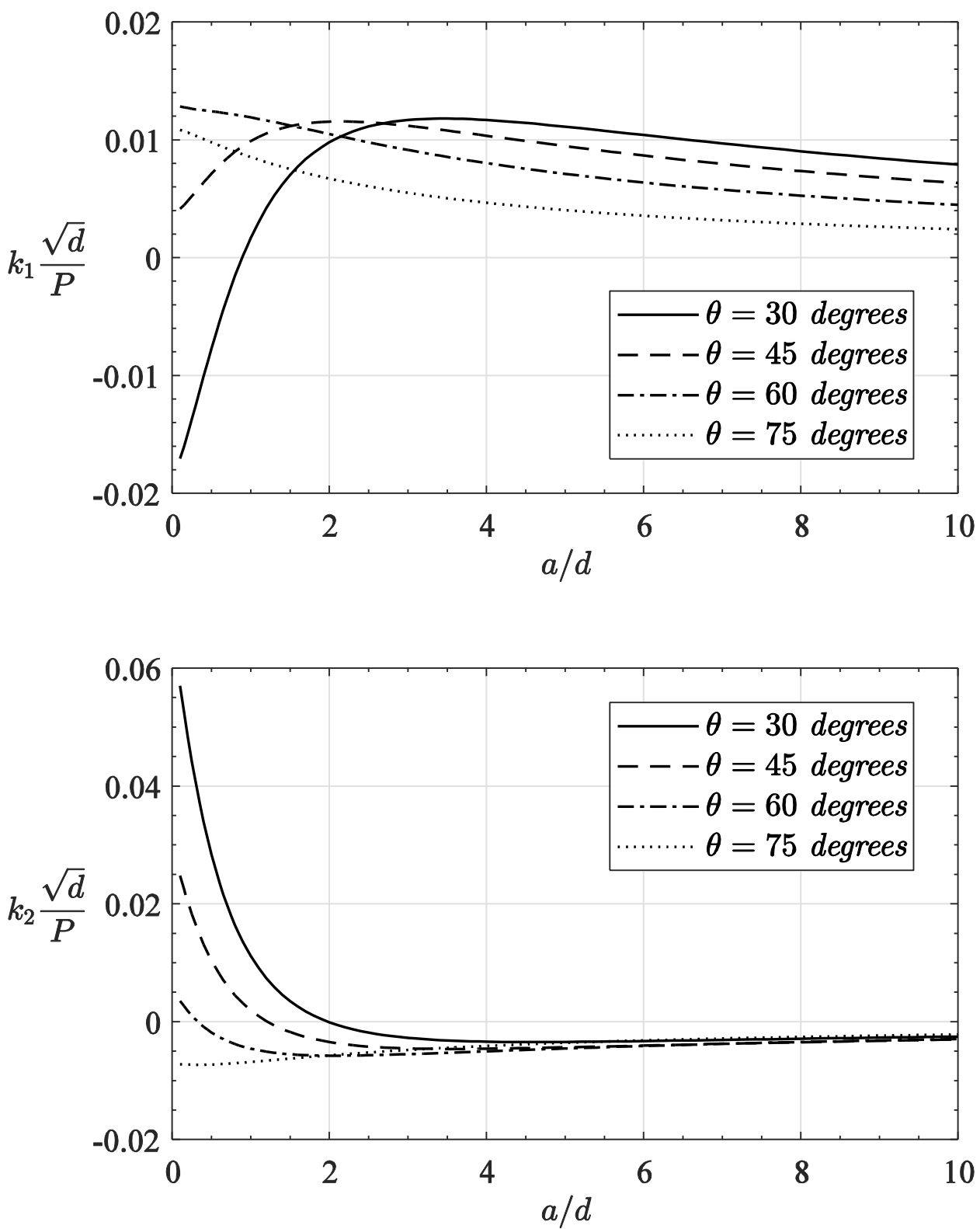

Figure 3.33 Mixed Mode Stress Intensity Factors for Inclined Edge Crack in Orthotropic Half-Plane of Plasma Sprayed Alumina Loaded by Circular Punch for Plane Strain Case and Fully Open Crack Assumption, $(b-a) / d=1, \eta_{1}$ $=0.2$. 

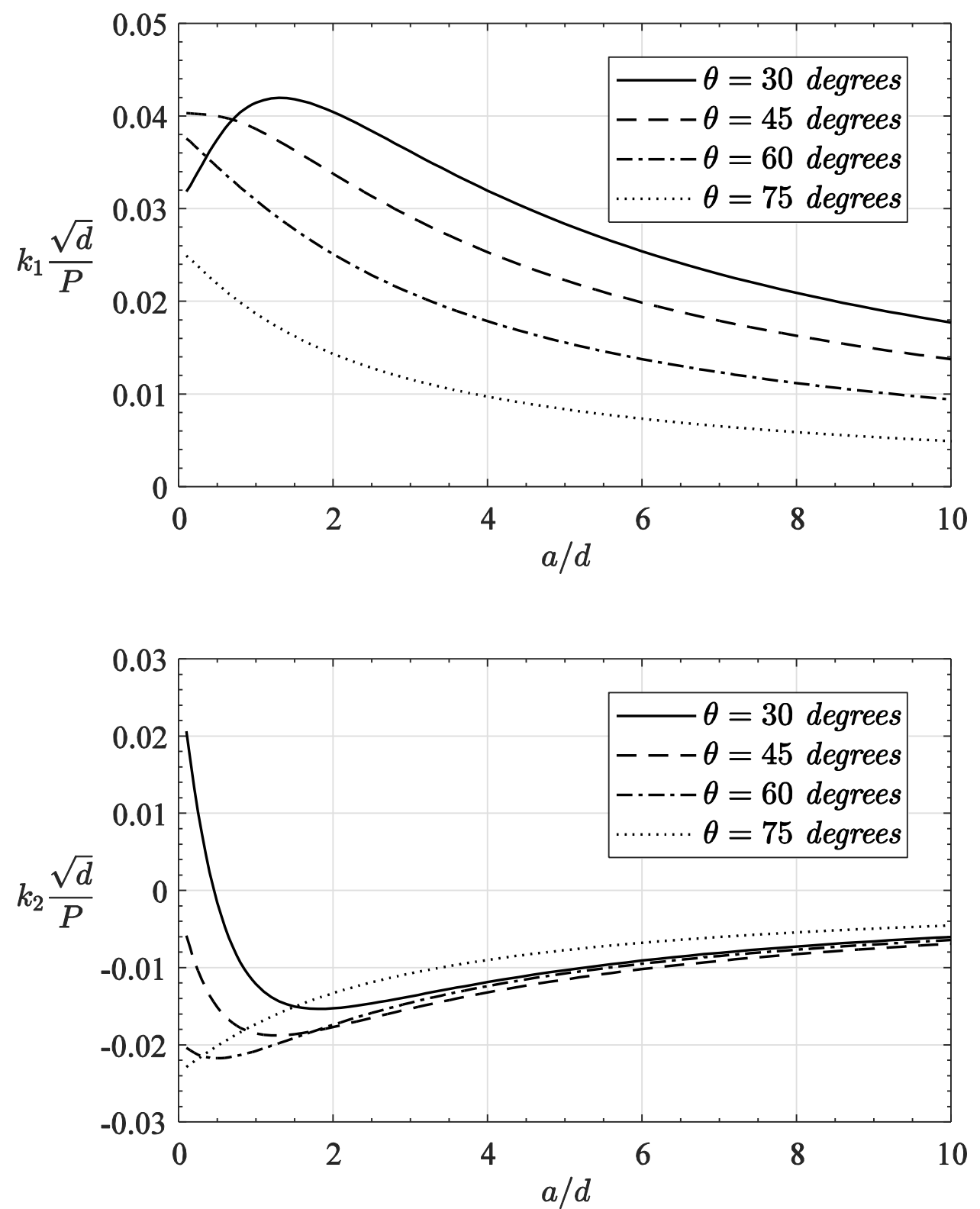

Figure 3.34 Mixed Mode Stress Intensity Factors for Inclined Edge Crack in Orthotropic Half-Plane of Plasma Sprayed Alumina Loaded by Circular Punch for Plane Strain Case and Fully Open Crack Assumption, $(b-a) / d=1, \eta_{1}$ $=0.4$. 

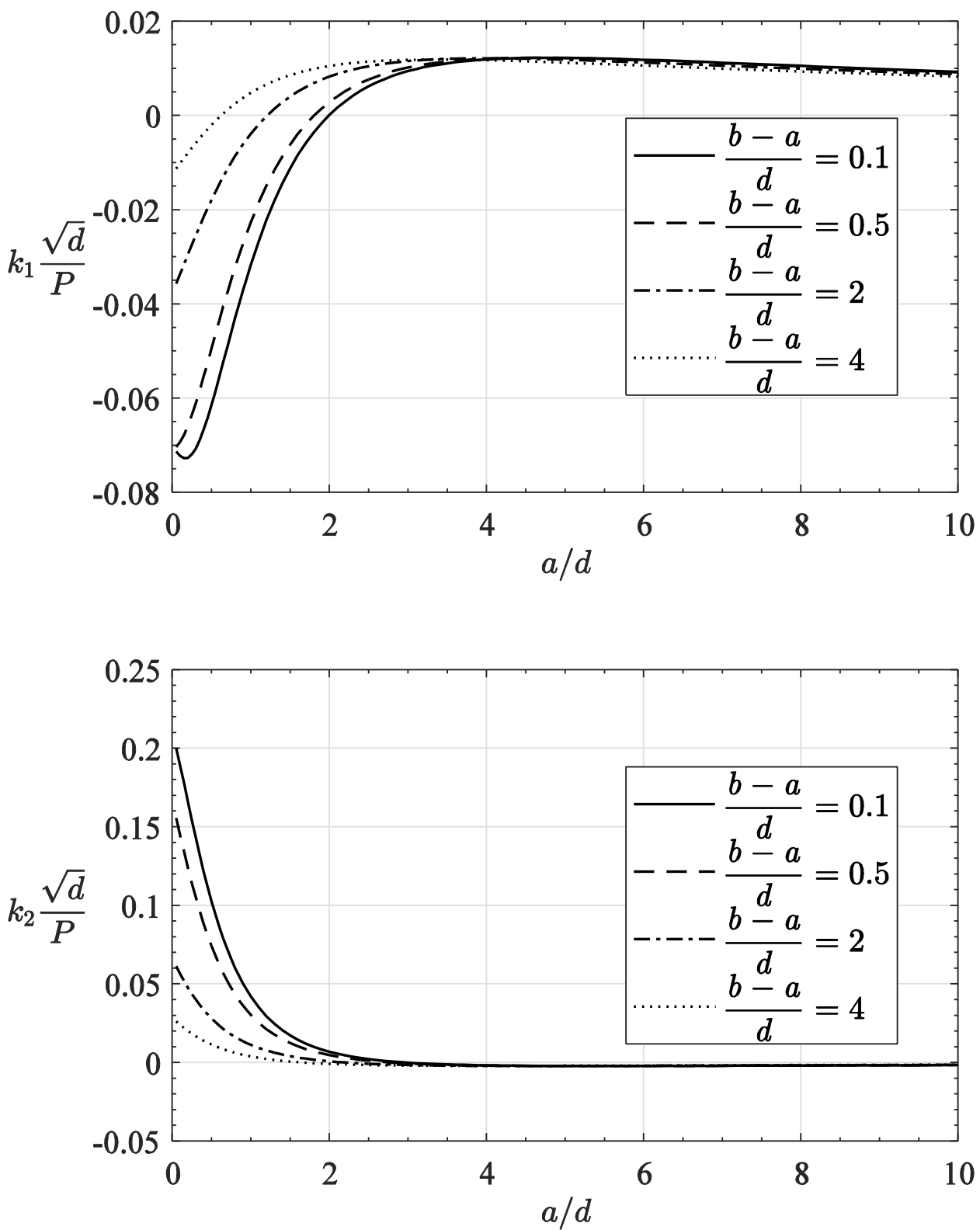

Figure 3.35 Effect of $(b-a) / d$ Variation on Mixed Mode Stress Intensity Factors for Inclined Edge Crack in Orthotropic Half-Plane of Plasma Sprayed Alumina Loaded by Circular Punch for Plane Strain Case and Fully Open Crack Assumption, Crack Angle $\theta=\pi / 12, \eta_{1}=0.2$. 

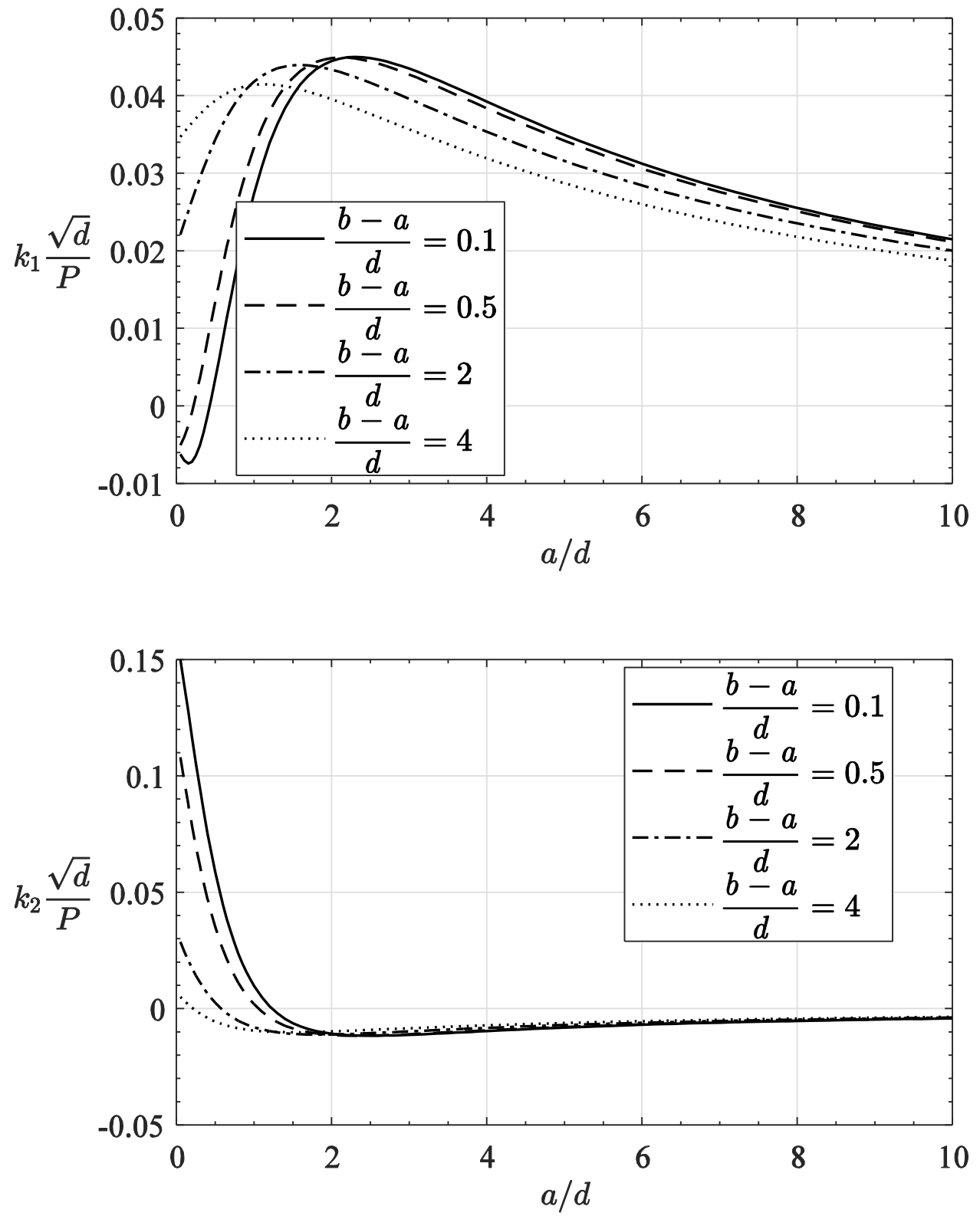

Figure 3.36 Effect of $(b-a) / d$ Variation on Mixed Mode Stress Intensity Factors for Inclined Edge Crack in Orthotropic Half-Plane of Plasma Sprayed Alumina Loaded by Circular Punch for Plane Strain Case and Fully Open Crack Assumption, Crack Angle $\theta=\pi / 12, \eta_{1}=0.4$. 

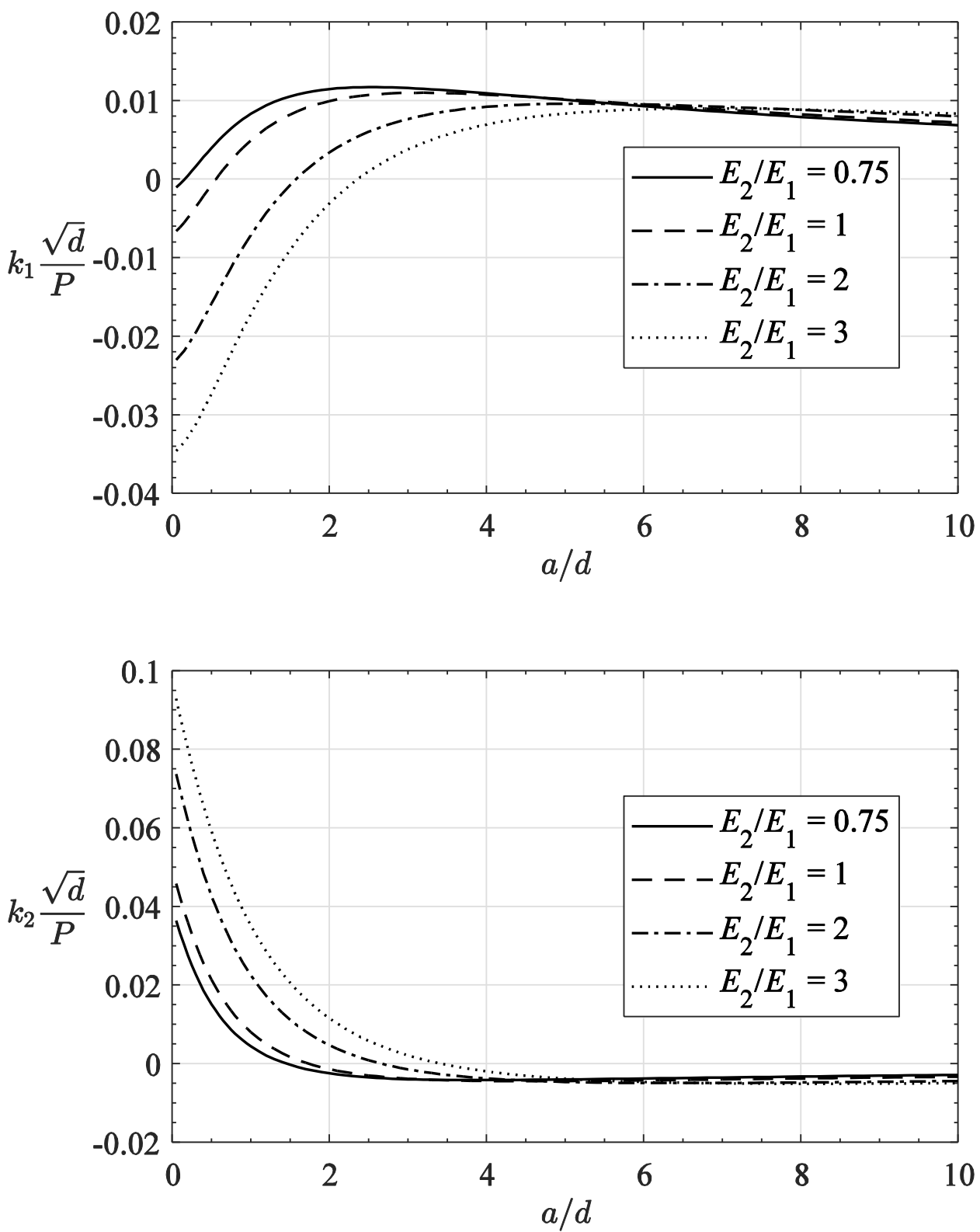

Figure 3.37 Effect of Elastic Modulus Ratio $E_{2} / E_{1}$ on Mixed Mode Stress Intensity Factors for Inclined Edge Crack in Orthotropic Half-Plane Loaded by Circular Punch for Plane Strain Case and Fully Open Crack Assumption, $(b-a) / d=1$, Crack Angle $\theta=2 \pi / 9, \eta_{1}=0.2$. 

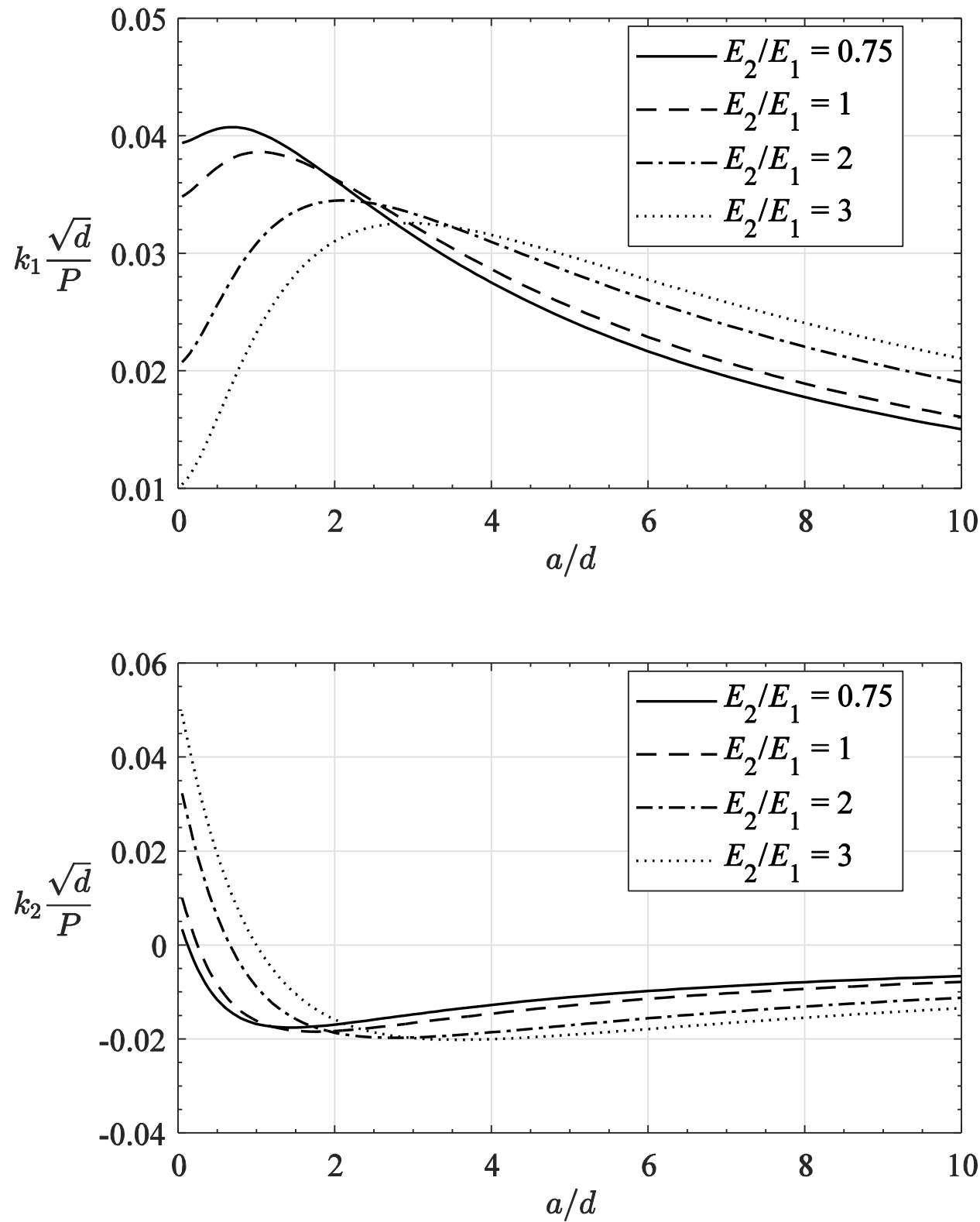

Figure 3.38 Effect of Elastic Modulus Ratio $E_{2} / E_{1}$ on Mixed Mode Stress Intensity Factors for Inclined Edge Crack in Orthotropic Half-Plane Loaded by Circular Punch for Plane Strain Case and Fully Open Crack Assumption, $(b-a) / d=1$, Crack Angle $\theta=2 \pi / 9, \eta_{1}=0.4$. 

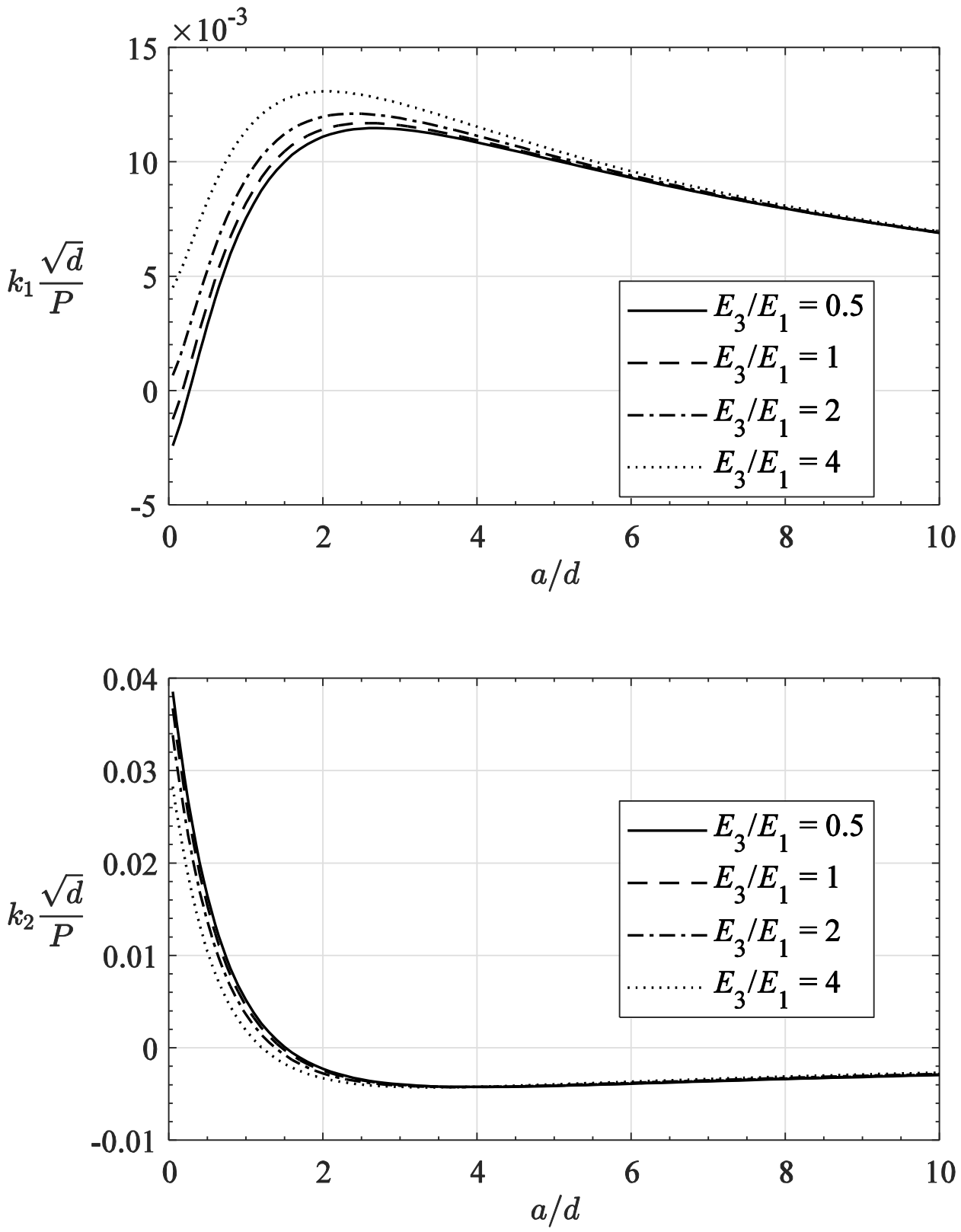

Figure 3.39 Effect of Elastic Modulus Ratio $E_{3} / E_{1}$ on Mixed Mode Stress Intensity Factors for Inclined Edge Crack in Orthotropic Half-Plane Loaded by Circular Punch for Plane Strain Case and Fully Open Crack Assumption, $(b-a) / d=1$, Crack Angle $\theta=2 \pi / 9, \eta_{1}=0.2$. 

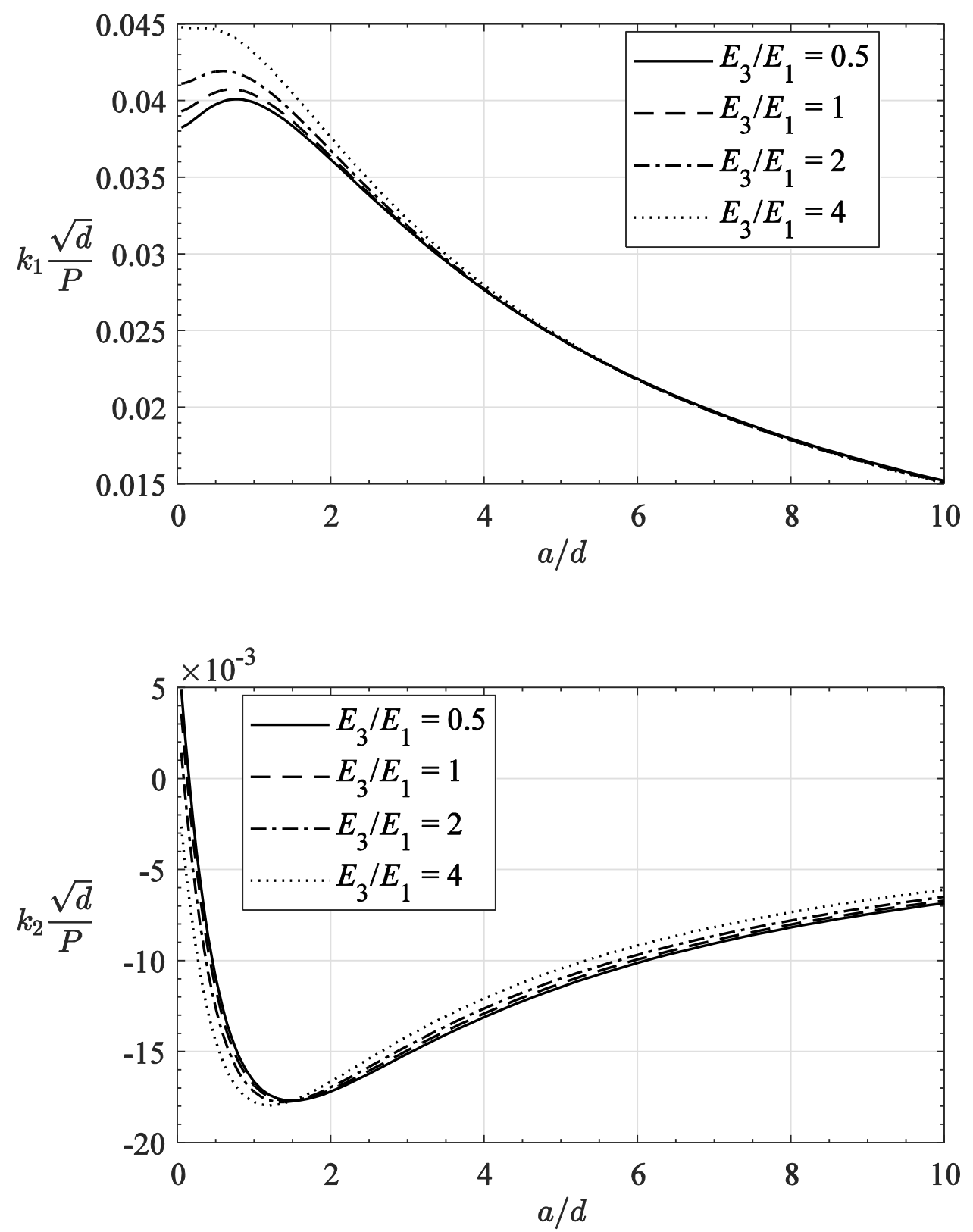

Figure 3.40 Effect of Elastic Modulus Ratio $E_{3} / E_{1}$ on Mixed Mode Stress Intensity Factors for Inclined Edge Crack in Orthotropic Half-Plane Loaded by Circular Punch for Plane Strain Case and Fully Open Crack Assumption, $(b-a) / d=1$, Crack Angle $\theta=2 \pi / 9, \eta_{1}=0.4$. 

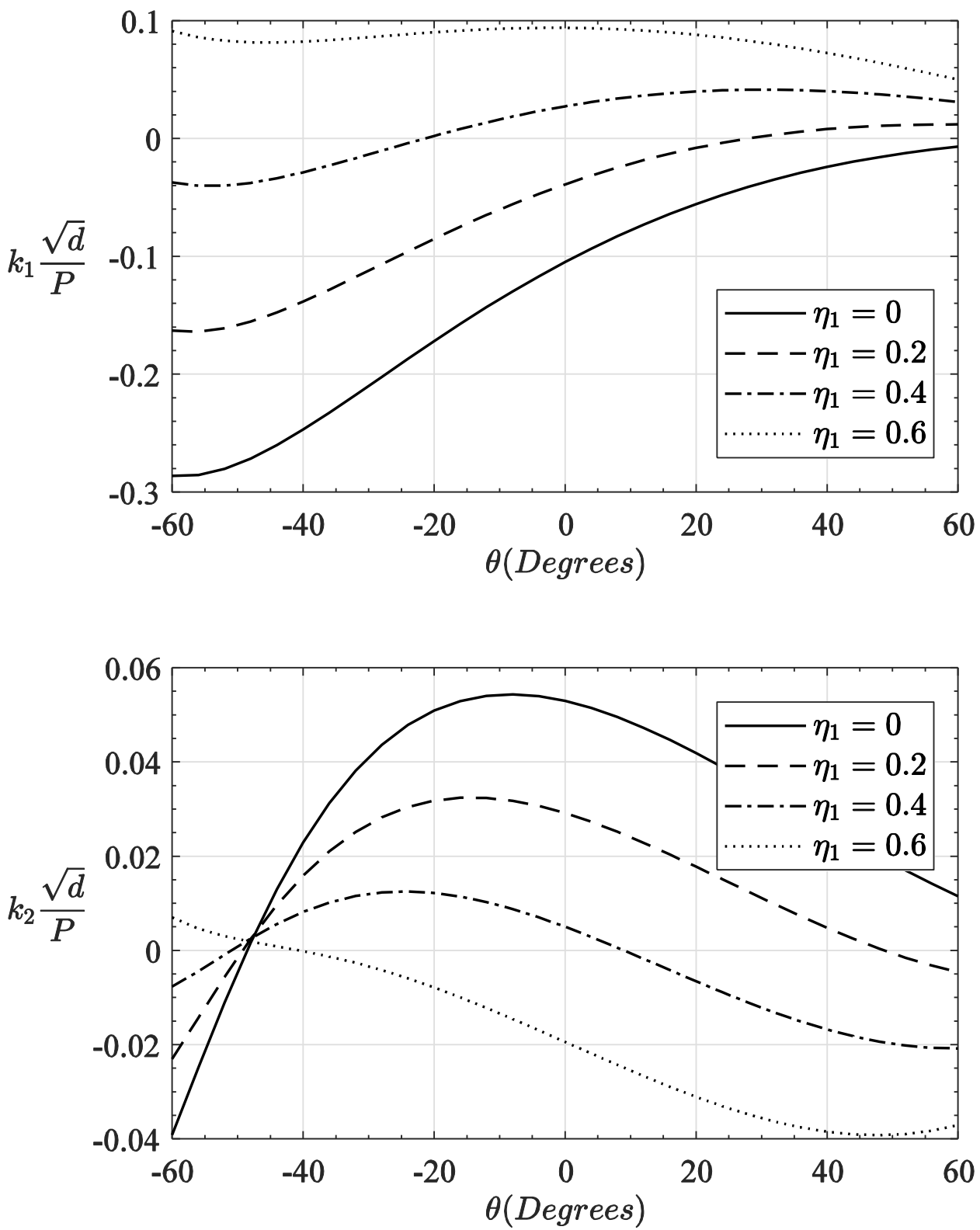

Figure 3.41 Effect of Crack Angle Variation on Mixed Mode Stress Intensity Factors for Inclined Edge Crack in Orthotropic Half-Plane of Plasma Sprayed Alumina Loaded by Circular Punch for Plane Strain Case and Fully Open Crack Assumption, $(b-a) / d=1, a / d=1$. 

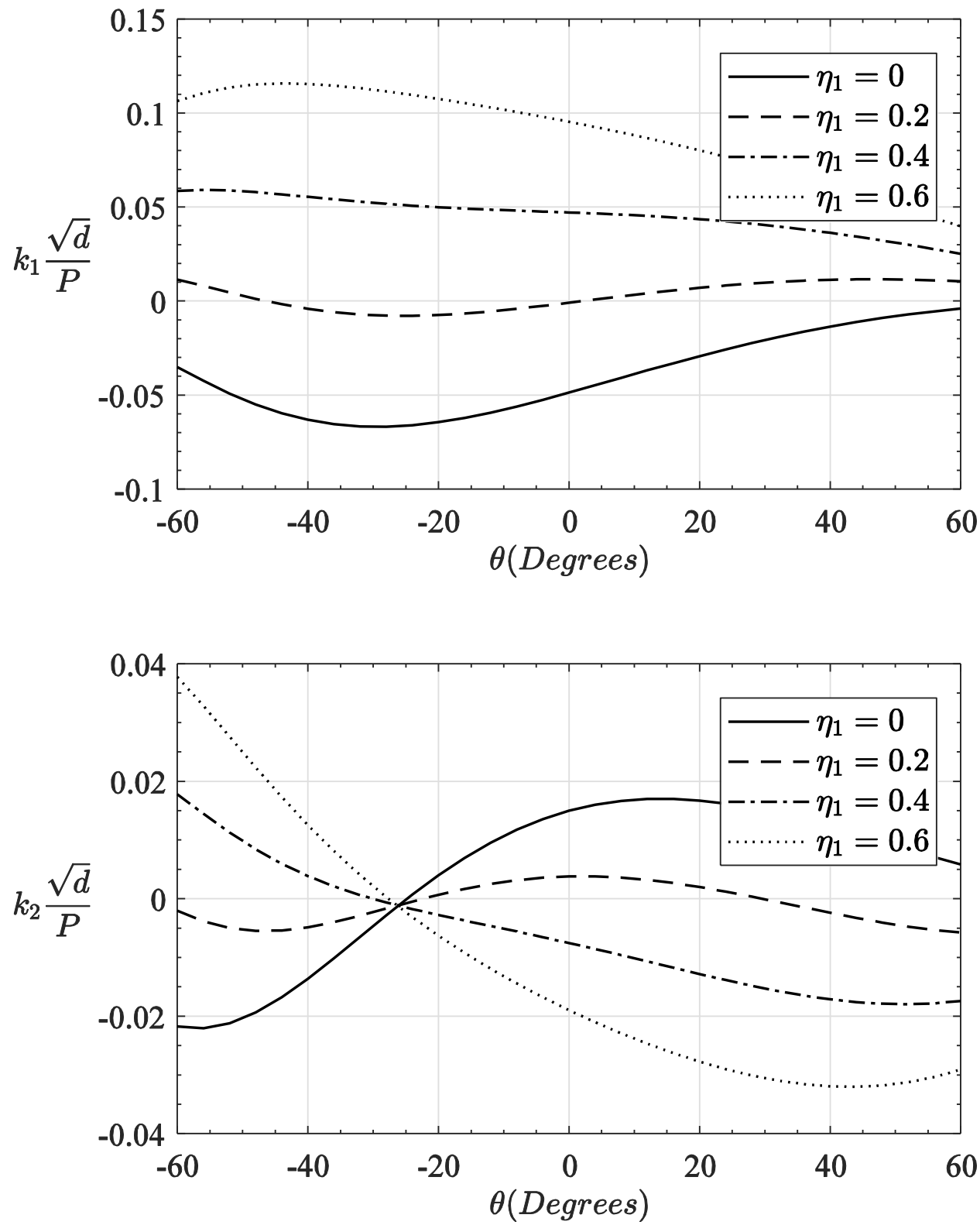

Figure 3.42 Effect of Crack Angle Variation on Mixed Mode Stress Intensity Factors for Inclined Edge Crack in Orthotropic Half-Plane of Plasma Sprayed Alumina Loaded by Circular Punch for Plane Strain Case and Fully Open Crack Assumption, $(b-a) / d=1, a / d=2$. 
3.4 Figures for Orthotropic Half-Plane Indented by Flat Stamp (Crack Tip and Full Closure Modes)
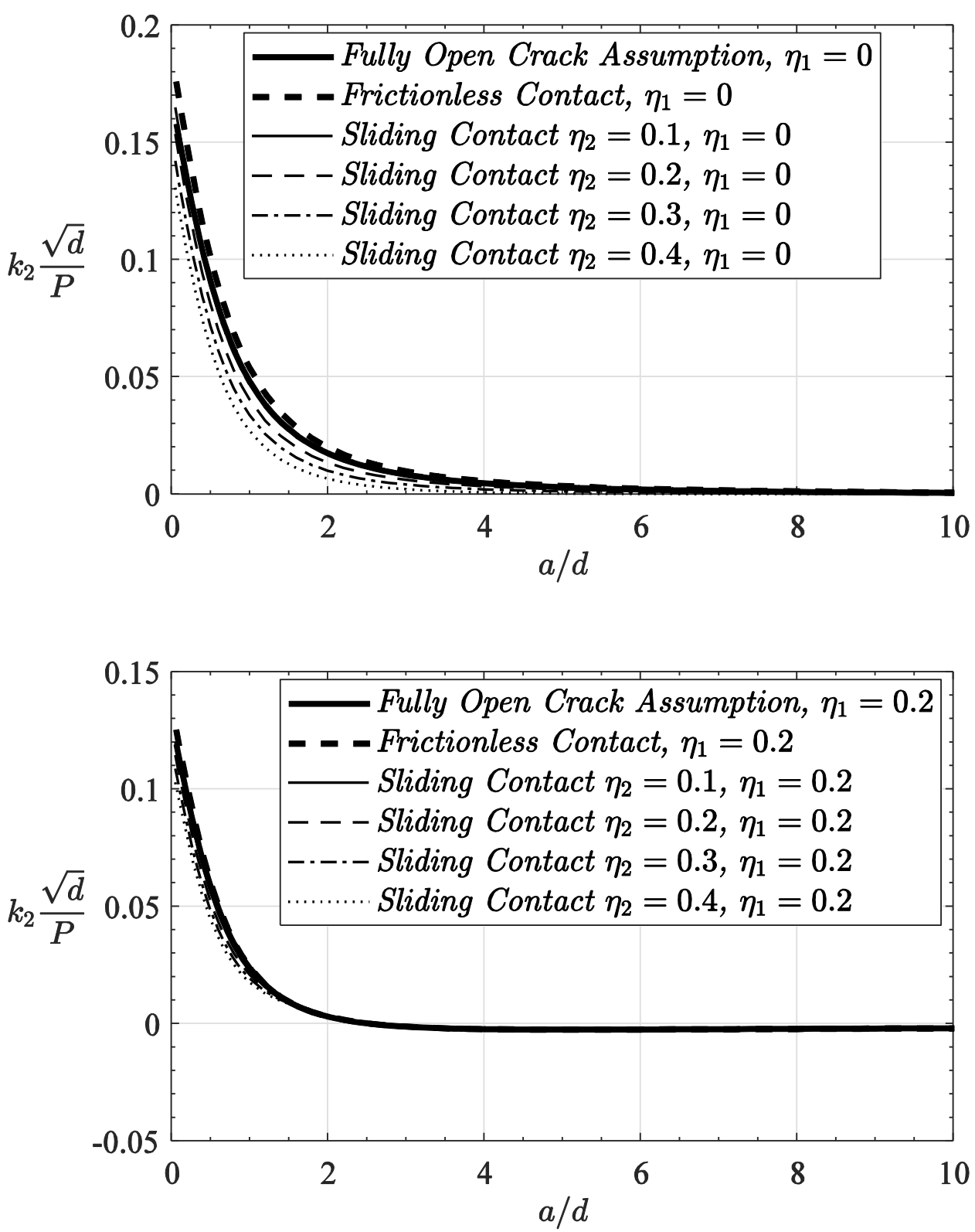

Figure 3.43 Effect of $\eta_{2}$ on Mode II SIF for Inclined Edge Crack in Orthotropic Half-Plane of Plasma Sprayed Alumina Loaded by Flat Punch for Plane Strain Case, $(b-a) / d=1$, Crack Angle $\theta=\pi / 12$. 

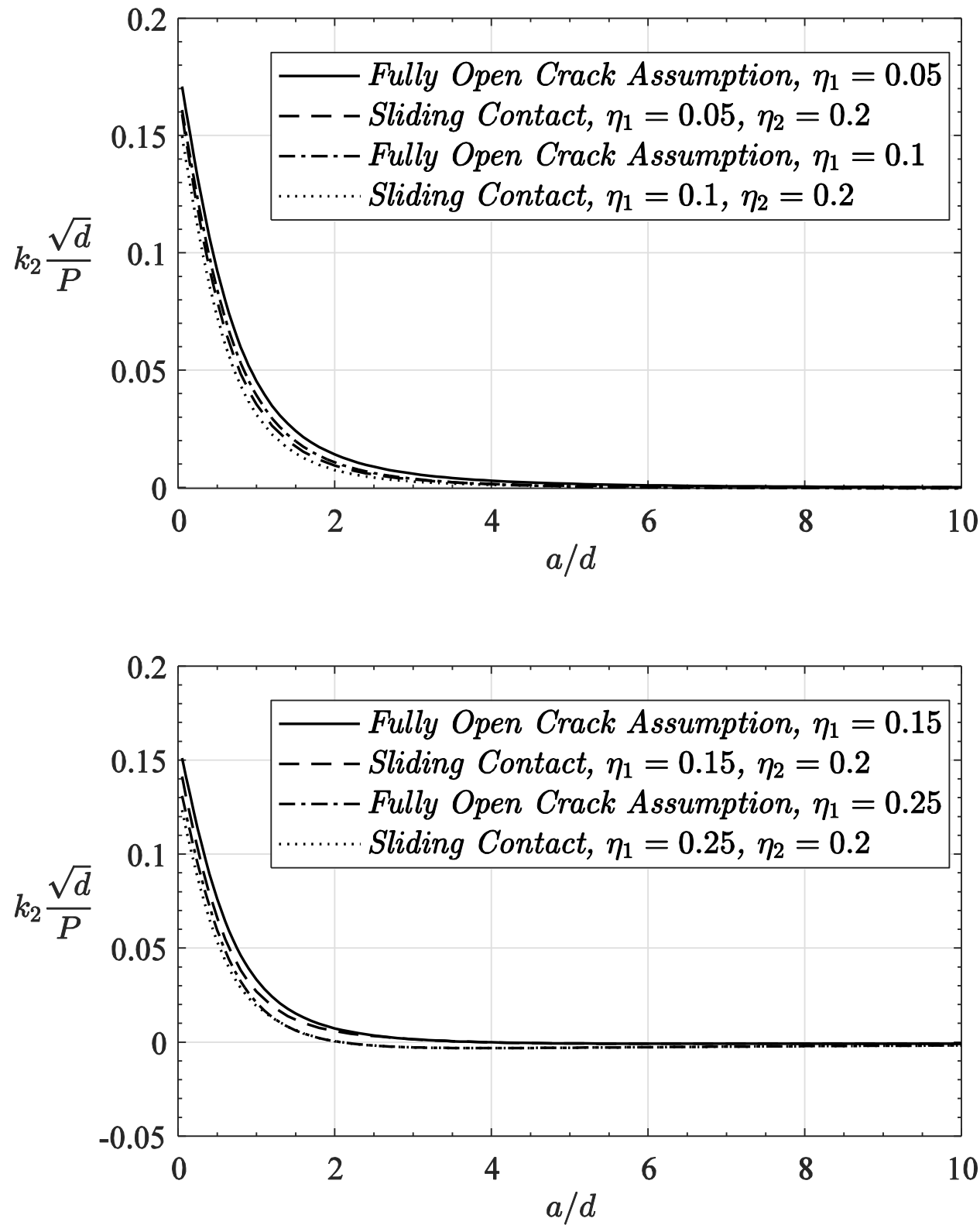

Figure 3.44 Effect of $\eta_{1}$ on Mode II SIF for Inclined Edge Crack in Orthotropic Half-Plane of Plasma Sprayed Alumina Loaded by Flat Punch for Plane Strain Case, $(b-a) / d=1$, Crack Angle $\theta=\pi / 18$. 

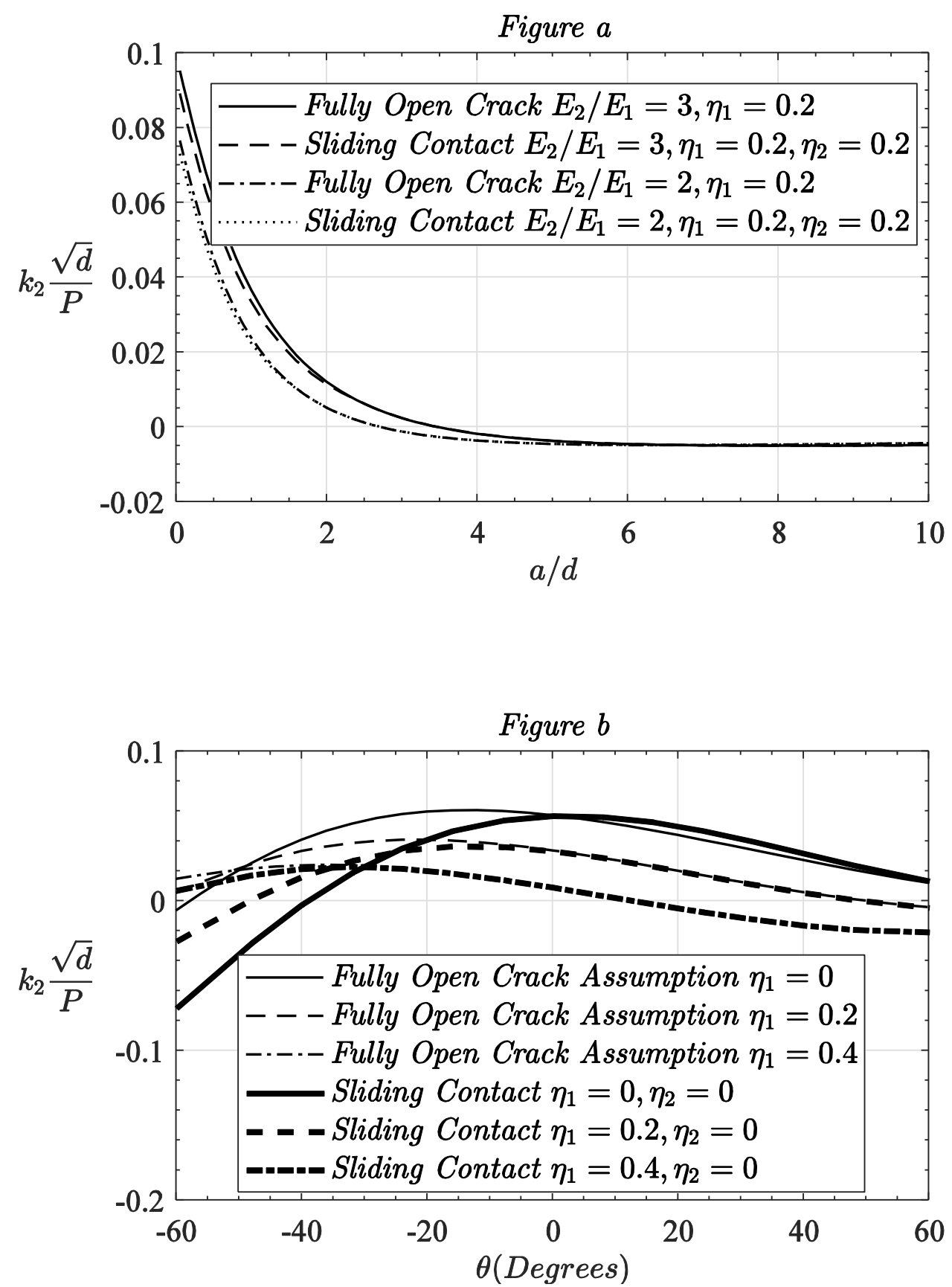

Figure 3.45 a) Effect of Elastic Modulus Ratio $E_{2} / E_{1}$ for $\theta=2 \pi / 9$ b) Effect of Crack Angle Variation on Mode II SIF's for Inclined Edge Crack in Orthotropic Half-Plane Loaded by Flat Punch for Plane Strain Case, $(b-a) / d=1$. 

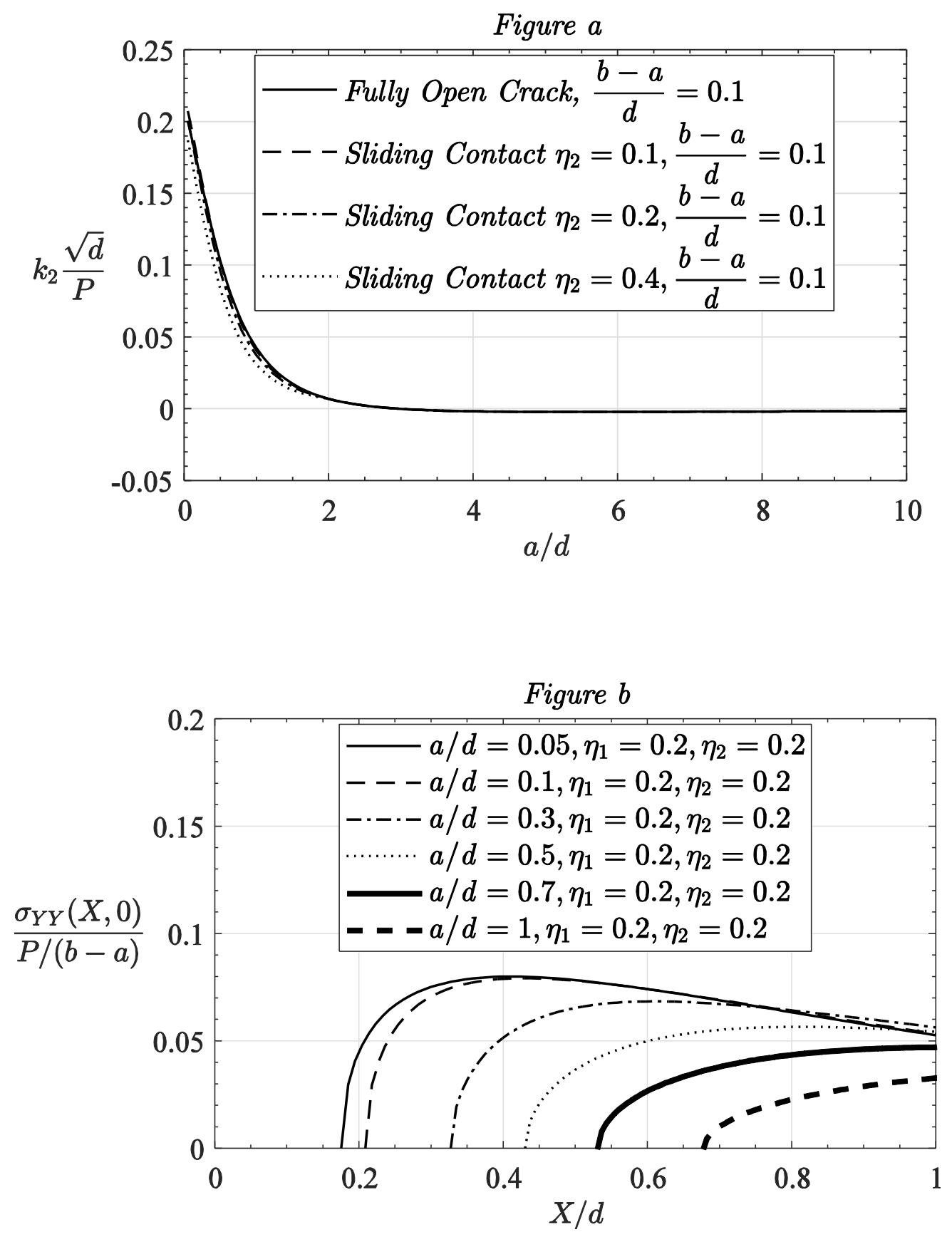

Figure 3.46 a) Effect of $\eta_{2}$ on Mode II SIF for $\left.(b-a) / d=0.1, b\right)$ Effect of $a / d$ Variation on Contact Pressure Distributions Between Crack Faces for Inclined Edge Crack in Orthotropic Half-Plane of Plasma Sprayed Alumina Loaded by Flat Punch for Plane Strain Case, Crack Angle $\theta=\pi / 12$ 

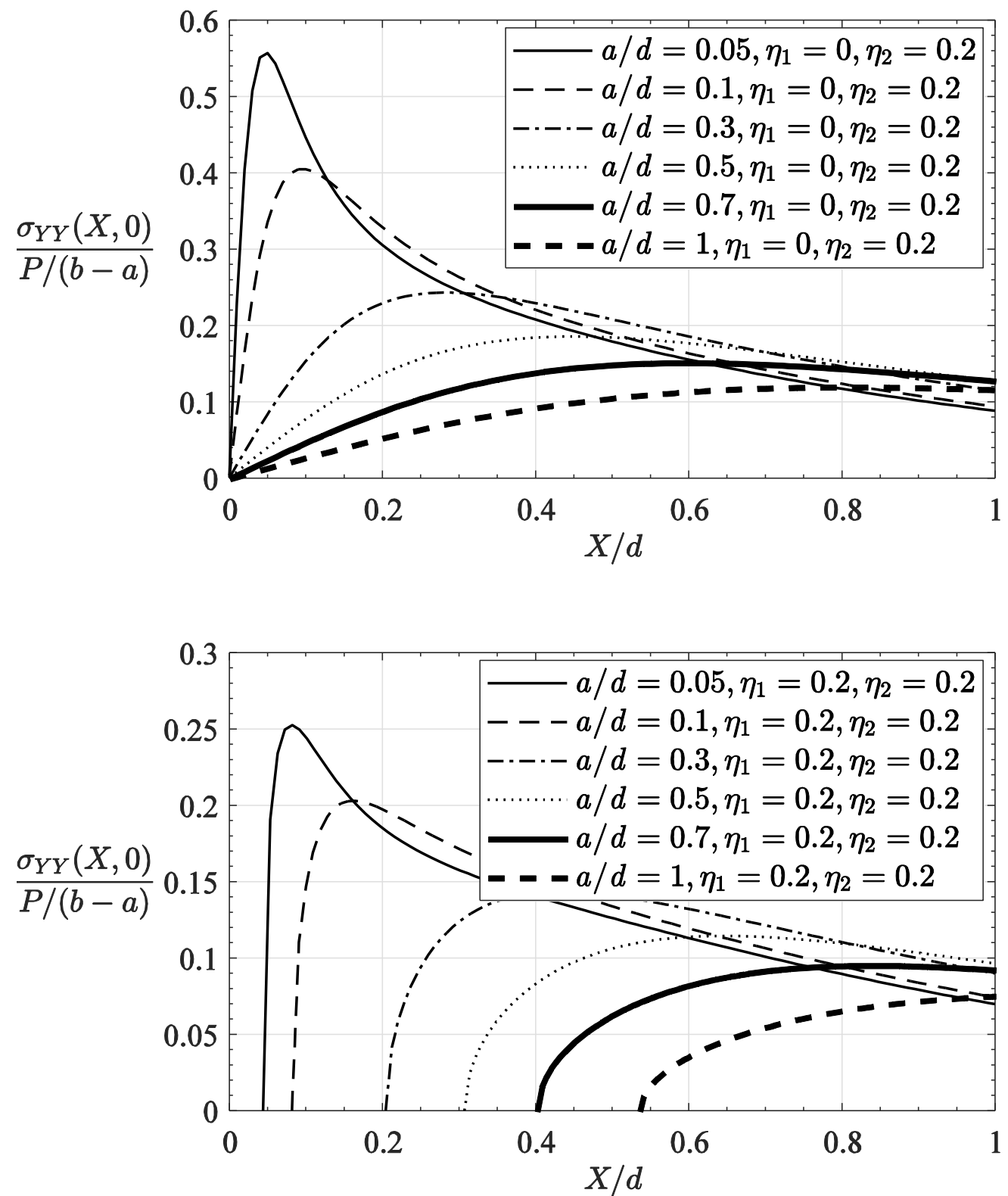

Figure 3.47 Effect of $a / d$ Variation on Contact Pressure Distributions Between Crack Faces for Straight Edge Crack in Orthotropic Half-Plane of Plasma Sprayed Alumina Loaded by Flat Punch for Plane Strain Case. 

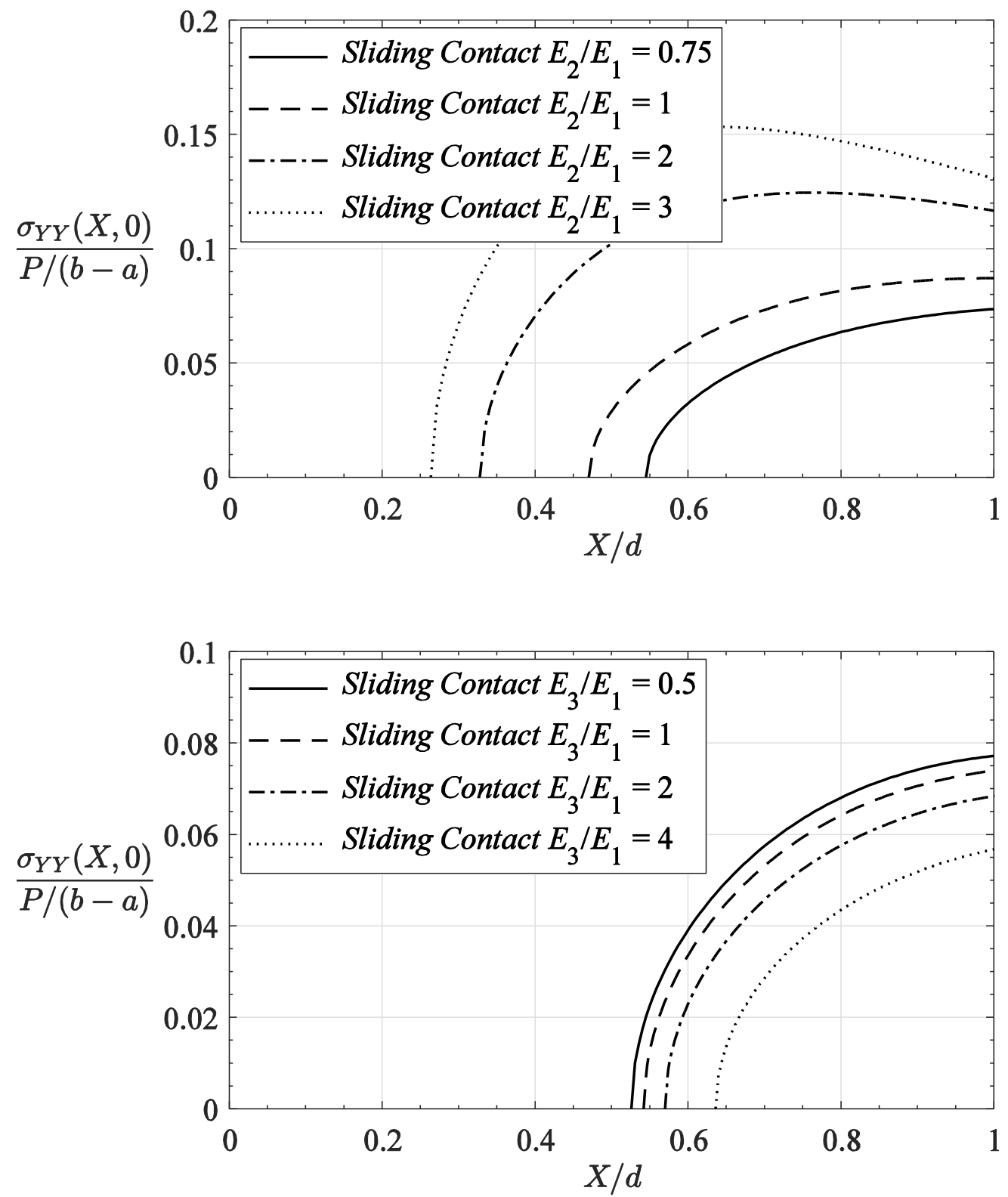

Figure 3.48 Effect of Elastic Modulus Ratios $E_{2} / E_{1}$ and $E_{3} / E_{1}$ on Contact Pressure Distributions Between Crack Faces for Straight Edge Crack in Orthotropic Half-Plane Loaded by Flat Punch for Plane Strain Case, $a / d=1, \eta_{1}=$ $0.2, \eta_{2}=0$. 

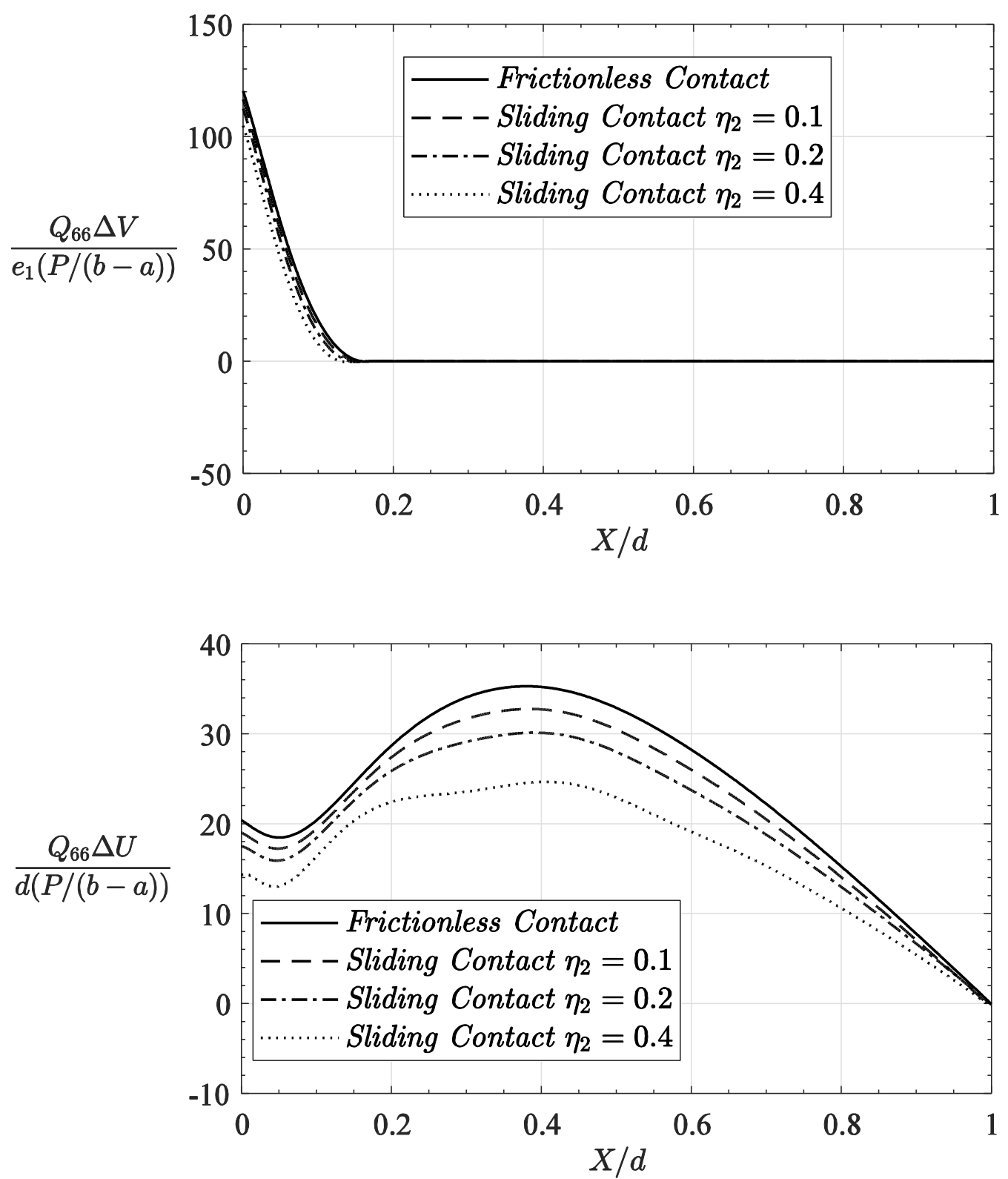

Figure 3.49 Effect of $\eta_{2}$ on Normalized Relative Crack Surface Displacements for Inclined Edge Crack in Orthotropic Half-Plane of Plasma Sprayed Alumina Loaded by Flat Punch for Plane Strain Case, Crack Angle $\theta=\pi / 12, a / d=0.25$, $(b-a) / d=1, \eta_{1}=0.1$. 


\section{CHAPTER 4}

\section{CONCLUSIONS AND FUTURE WORK}

\subsection{Conclusions}

In the present study, a solution method for the calculation of stress intensity factors is proposed for inclined edge cracks in orthotropic half-plane exposed to surface loads originating from the contact of rigid punches. It is found that $\eta_{1}$ is the dominant parameter in the determination of whether fully open crack assumption is valid or not. For large enough values of $\eta_{1}$, fully open crack assumption is confirmed by positive mode I stress intensity factors $\left(k_{1}>0\right)$. Based on the iterative solution of singular integral equations, the crack closure is also taken into consideration. Iterative solution for determination of closed parts is a very time consuming process compared to the solution by fully open crack assumption because of several repetitions of the expansion and collocation methods. For this reason, the results for crack closure are presented only for cracks in orthotropic half-plane loaded by flat punch.

Computer programs are designed by using MAPLE in order to carry out all numerical procedures described in Chapter 2 and Chapter 3. Various sets of results about crack angle variation, $\mathrm{CoF}$ at the material surface and between crack faces, the ratios of Young's moduli of the material, contact loading size - crack length ratio are given for cracks under the effect of indentation of three loading punch profiles in Chapter 3. Besides, cracks exposed to uniform tension and bending load in the study by Beghini et al [43] are also examined in Chapter 3 for 
verification. The results of the present study for both open and closed cracks are validated by comparing them with the ones in the literature.

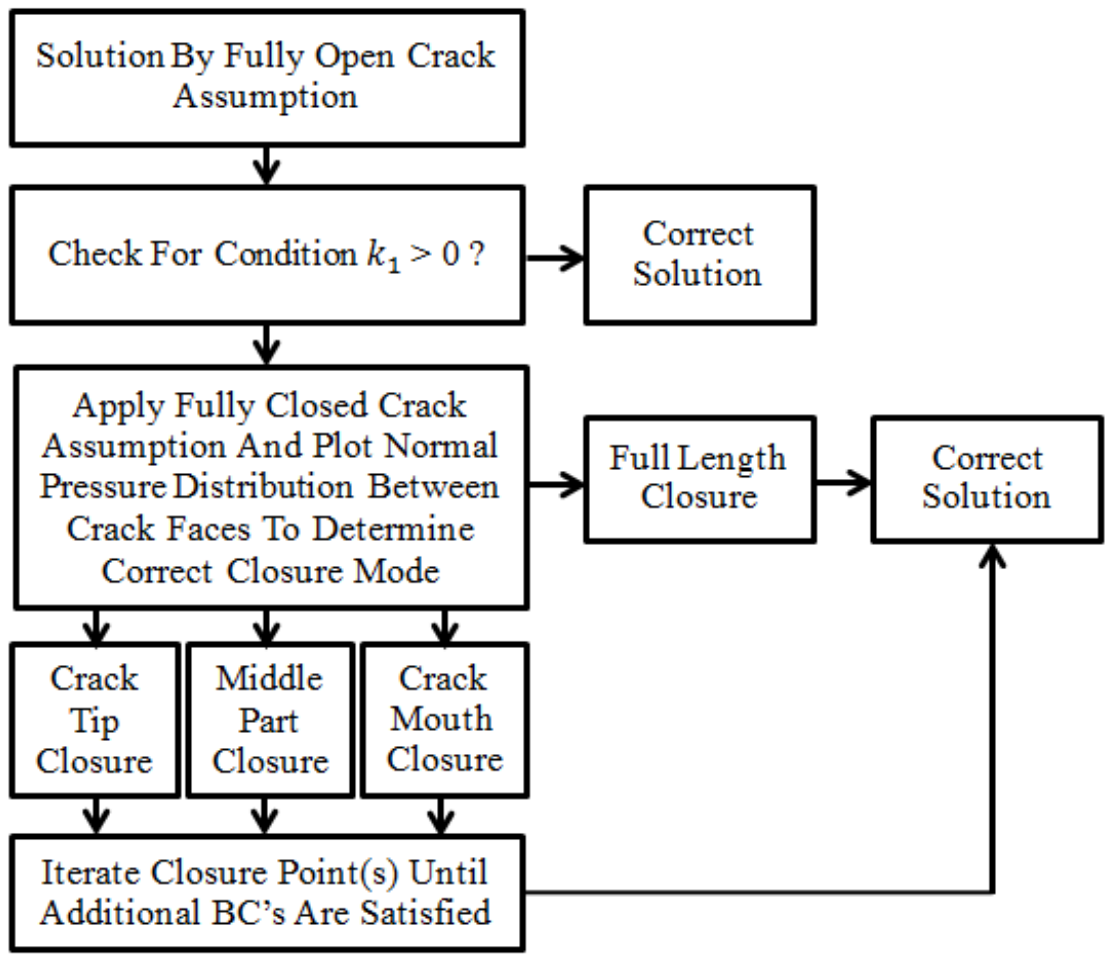

Figure 4.1 General Solution Algorithm

General solution algorithm used in the present study is given in Figure 4.1. First, the solution always starts with fully open crack assumption. Then, the results are judged by using mode I stress intensity factors obtained by fully open crack analysis. If mode I stress intensity factors are positive, correct solution is reached. Otherwise, further analyses are performed. Normal pressure distributions between crack faces are plotted by applying full length closure mode. Following the data on contact pressure distribution between crack faces, the suitable closure mode is 
determined. If contact pressure distribution between crack faces is found as compression for all points, then full length closure assumption is confirmed. On the other hand, if crack segments under the effect of tension are seen, it means that those segments are not in contact [41]. Then, closure point(s) are iterated until additional boundary condition(s) stemmed from crack closure are satisfied.

The outcomes of the study are summarized as follows:

- Effect of elastic modulus ratio $E_{2} / E_{1}$ on mixed mode stress intensity factors for a crack under uniform tension is illustrated in Figure 3.3. As $E_{2} / E_{1}$ ratio goes up, a notable decrease in mode I stress intensity factors and a notable increase in mode II stress intensity factors are observed.

- When the shear stress acting on the material surface equal to zero $\left(\eta_{1}=\right.$ $0)$, negative mode I stress intensity factors are obtained for all punch locations as shown in Figure 3.10, Figure 3.21 and Figure 3.32 for flat, triangular and circular punches, respectively. It means that crack closure occurs independent of punch location for $\eta_{1}=0$. As $\mathrm{CoF}$ at the material surface increases, mode I stress intensity factors become positive. In other words, tensile stresses exist at the crack tips and fully open crack assumption is valid in those cases.

- The effect of $a / d$ ratio on mixed mode stress intensity factors is given with respect to crack angle variation between $30^{\circ}$ and $75^{\circ}$ in Figure 3.11Figure 3.12, Figure 3.22-Figure 3.23 and Figure 3.33-Figure 3.34. Crack closure is not observed for the cases having crack angle higher than $45^{\circ}$.

- As $(b-a) / d$ ratio decreases, mixed mode stress intensity factor curves shift right. (Check Figure 3.13-Figure 3.14, Figure 3.24-Figure 3.25 and Figure 3.35-Figure 3.36). Due to this shift, $k_{1}$ decreases for low $a / d$ ratio, 
while $k_{2}$ increases. On the other hand, $(b-a) / d$ ratio causes minor changes for cases with high $a / d$ ratio.

- As $E_{2} / E_{1}$ ratio increases, perceptible decrements in mode I stress intensity factors and increments in mode II stress intensity factors can be observed for low $a / d$ ratios. (Check Figure 3.15-Figure 3.16, Figure 3.26-Figure 3.27 and Figure 3.37-Figure 3.38). While the variation in $E_{2} / E_{1}$ ratio causes inconsiderable changes for cases with high $a / d$ ratio and $\eta_{1}=0.2$, it results in more significant changes for cases with high $a / d$ ratio and $\eta_{1}=0.4$.

- The influence of $E_{3} / E_{1}$ ratio on mixed mode stress intensity factors is inconsiderable for almost all cases. A slight increase in mode I stress intensity factors and a slight decrease in mode II stress intensity factors can still be observed as $E_{3} / E_{1}$ ratio goes up. (Check Figure 3.17-Figure 3.18, Figure 3.28-Figure 3.29 and Figure 3.39-Figure 3.40)

- The effect of crack angle variation between $-60^{\circ}$ and $60^{\circ}$ on mixed mode stress intensity factors is given with respect to $\eta_{1}$ in Figure 3.19-Figure 3.20, Figure 3.30-Figure 3.31 and Figure 3.41-Figure 3.42. The highest and lowest values for mixed mode stress intensity factors due to crack angle variation can be checked in these figures.

- Crack tip and full closure modes are investigated in Section 3.4. For those cases, mode I stress intensity factor vanishes. A slight decrease in mode II stress intensity factors is observed for all cases when they are modified with the crack tip closure as shown in Figure 3.43, Figure 3.44 and Figure 3.46a. Because, the friction between crack faces reduces the shear stress at the crack tip. However, the change in mode II stress intensity factors 
becomes more critical for inclined cracks with negative crack angles as shown in Figure 3.45b.

- Contact pressure distributions between crack faces caused by the indentation of flat punch are given for several cases in Figure 3.46b, Figure 3.47 and Figure 3.48. For $\eta_{1}=0$ and $\eta_{2}=0.2$, complete closure mode is observed for all $a / d$ values. For $\eta_{1}=0.2$ and $\eta_{2}=0.2$, crack tip closure mode is detected. Closure length and total contact pressure decrease as $a / d$ ratio goes up.

- Effects of both $E_{2} / E_{1}$ and $E_{3} / E_{1}$ ratios on contact pressure distributions between crack faces for crack tip closure mode are illustrated in Figure 3.48. As $E_{2} / E_{1}$ ratio increases and $E_{3} / E_{1}$ ratio decreases, closure length and total contact pressure get larger.

- Finally, normalized crack surface displacements due to flat punch indentation are given for crack tip closure mode in Figure 3.49. Note that crack surface displacements in tangential direction for this case result in forward slipping of crack faces. Thus, the sign of the friction between crack faces should be chosen accordingly.

\subsection{Future Work}

Investigated crack closure modes in this study contain a single closed portion. However, more than a single closed portion may be encountered under more complex loading condition as shown in Figure 4.2. The formulation can be updated for other closure modes considering two or more closed portions as the future study.

In this study, it is assumed that crack faces slide over each other in the whole closed region of the crack for small values of coefficient of friction between the crack faces. However, sticking of crack faces are expected for larger values of $\eta_{2}$ 
as shown in Figure 4.3 and Figure 4.4. Thus, sticking crack face contact problem can be examined as the future study. If the sliding crack face condition is violated at $X=d$, there will not be any stress intensification at the crack tip. Hence, the crack is locked and it cannot propagate.

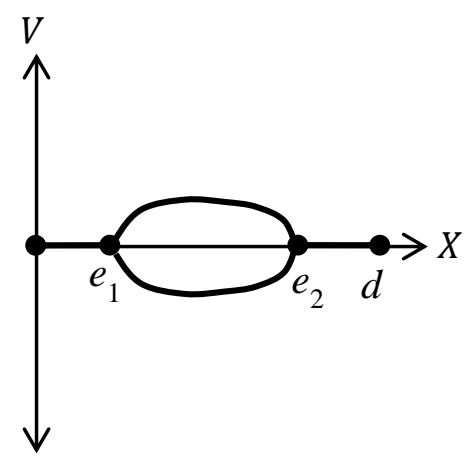

Crack Mouth and Tip are Closed

Middle Part Open

Figure 4.2 An Example of Other Closure Modes

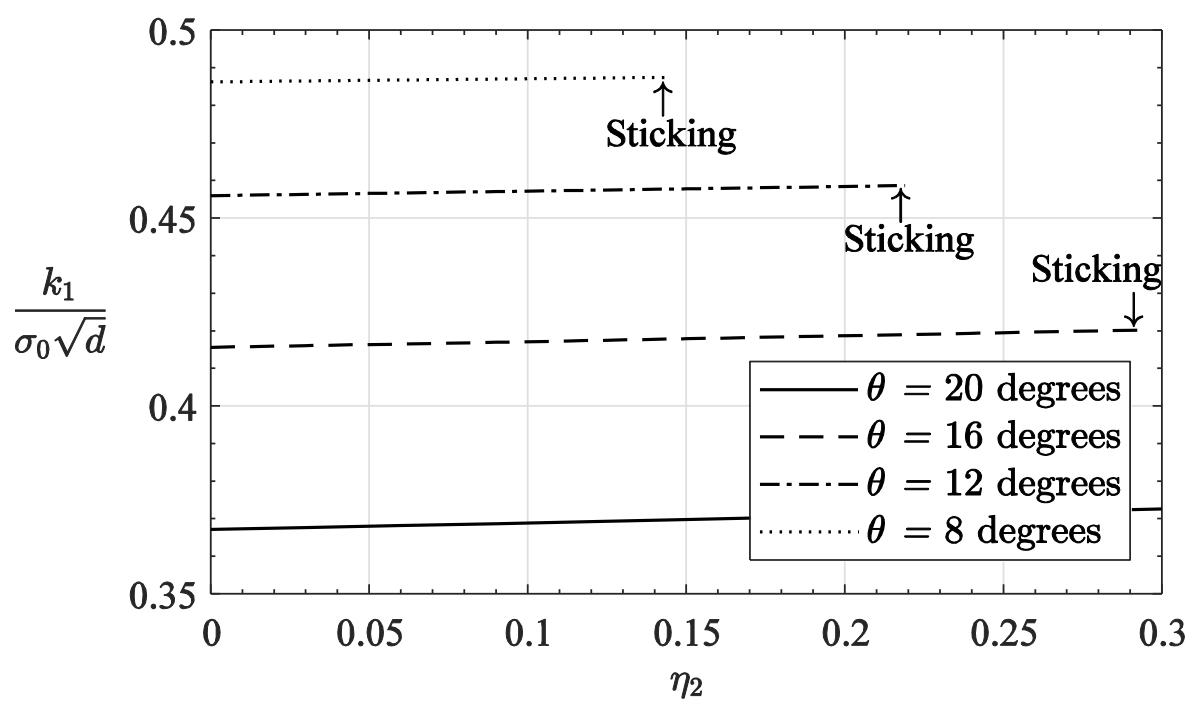

Figure 4.3 Effect of $\eta_{2}$ on Sticking of Crack Faces for Inclined Edge Cracks under Bending Load for Plane Strain Case. (Mode I SIF's) 


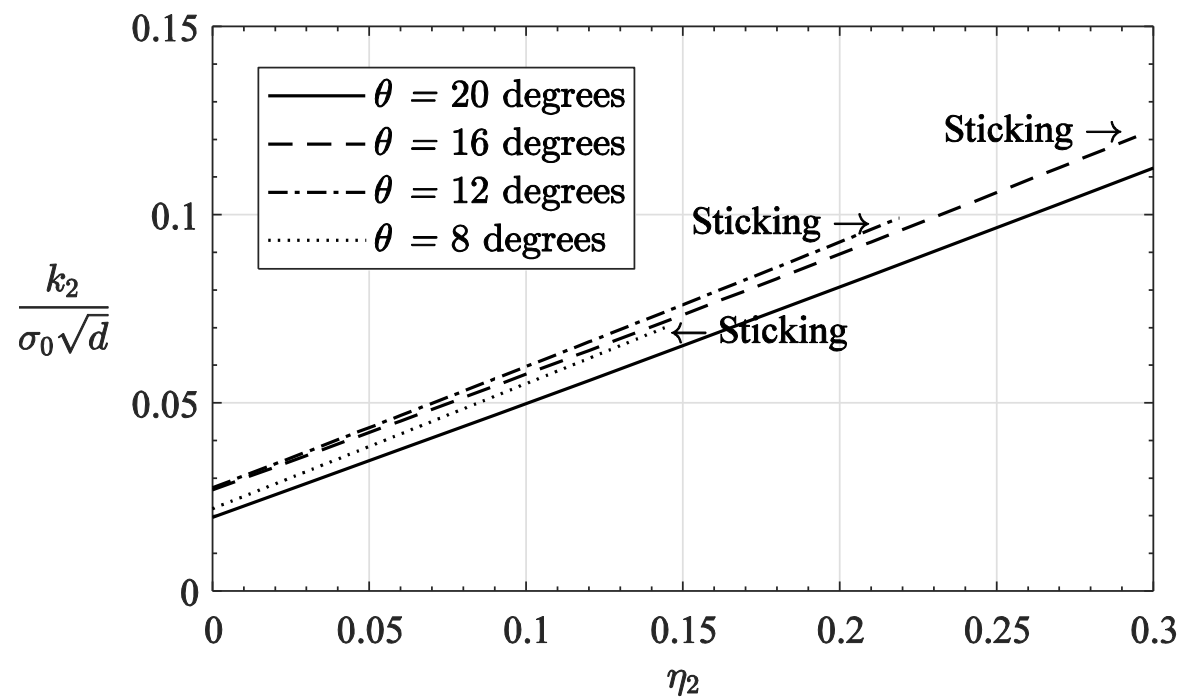

Figure 4.4 Effect of $\eta_{2}$ on Sticking of Crack Faces for Inclined Edge Cracks under Bending Load for Plane Strain Case. (Mode II SIF's)

For sticking of crack faces, influence of $\eta_{2}$ on SIF's are illustrated in Figure 4.3 and Figure 4.4. The maximum coefficients of friction are found for cracks under bending load as $0.145,0.219$ and 0.294 for $\theta=8^{\circ}, \theta=12^{\circ}$ and $\theta=16^{\circ}$, respectively before sticking starts. In other words, sliding crack face condition is violated if $\eta_{2}$ is beyond these values. 


\section{REFERENCES}

[1] Dag, S., 2001, "Crack and Contact Problems in Graded Materials", Ph.D. Dissertation, Department of Mechanical Engineering and Mechanics, Lehigh University, Bethlehem, Pennsylvania, USA.

[2] Sarikaya, D., 2014, “A Surface Crack in an Orthotropic Medium Subjected to Sliding Contact by a Rigid Stamp", Ph.D. Dissertation, Department of Mechanical Engineering, Middle East Technical University, Ankara, Turkey.

[3] Delale, F. and Erdogan F., 1977, “The Problem of Internal and Edge Cracks in an Orthotropic Strip", NASA CR-145157.

[4] Li, X.-F. and Lee, K.Y., 2009, "Closed-Form Solution for an Orthotropic Elastic Strip with a Crack Perpendicular to the Edges under Arbitrary Antiplane Shear", Zeitschrift für Angewandte Mathematik und Mechanik, Vol.89, pp.370-382.

[5] Ding, S.-H., Zhou, Y.-T. and Li, X., 2014, "Interface Crack Problem in Layered Orthotropic Materials under Thermo-mechanical Loading", International Journal of Solids and Structures, Vol.51, pp.4221-4229.

[6] Ding, S.-H. and Li, X. 2014, "The Collinear Crack Problem for an Orthotropic Functionally Graded Coating-Substrate Structure", Archive of Applied Mechanics, Vol.84, pp.291-307.

[7] Dag, S. and Ilhan, K.A., 2008, "Mixed-Mode Fracture Analysis of Orthotropic Functionally Graded Material Coatings Using Analytical and 
Computational Methods", Journal of Applied Mechanics-Transactions of the ASME, Vol.75, paper no: 051104.

[8] Dag, S., Yildirim, B. and Sarikaya, D., 2007, "Mixed-Mode Fracture Analysis of Orthotropic Functionally Graded Materials under Mechanical and Thermal Loads", International Journal of Solids and Structures, Vol.44 pp.7816-7840.

[9] Zhou, Y.T., Lee, K.Y. and Jang, Y.H., 2014, "Indentation Theory on Orthotropic Materials Subjected to a Frictional Moving Punch”, Archives of Mechanics, Vol.66, pp.71-94.

[10] Guler, M.A., 2014, "Closed-Form Solution of the Two-Dimensional Sliding Frictional Contact Problem for an Orthotropic Medium", International Journal of Mechanical Sciences, Vol.87, pp.72-88.

[11] Dag, S., Guler, M.A., Yildirim, B. and Ozatag, C., 2008, "Sliding Frictional Contact between a Rigid Punch and a Laterally Graded Elastic Medium”, International Journal of Solids and Structures, Vol.46, pp.4038-4053.

[12] Dag, S., El-Borgi, S. and Erdogan F., 2005, "Partial Closure Analysis of a Surface Crack in a Graded Medium Loaded by a Frictional Flat Stamp", CD Proceedings of the 11th International Conference on Fracture.

[13] Beghini, M., Bertini, L. and Fontanari, V., 2003, "Analysis of a Partially Closed Oblique Edge Crack under Surface Travelling Load", Computational Fluid and Solid Mechanics, Elsevier Science Ltd, pp.87-90.

[14] Birinci, A. and Cakiroglu, F.L., 2003, "Partial Closure of a Crack Located in an Infinite Elastic Layer", European Journal of Mechanics A/Solids Vol.22, pp.583-590. 
[15] Kahya, V., Birinci, A., Ozsahin, T.S. and Erdol, R., 2006, "Partial closure of a crack located in an anisotropic infinite elastic layer", European Journal of Mechanics A/Solids, Vol.25 pp.819-833.

[16] Hills, D.A. and Nowell, D., 1989, "Stress Intensity Calibrations for Closed Cracks", Journal of Strain Analysis, Vol.24, No:1, pp.37-43.

[17] Hills D.A., Kelly, P.A., Dai, D.N. and Korsunsky, A.M., 1996, Solution of Crack Problems: The Distributed Dislocation Technique, Springer Science+Business Media Dordrecht.

[18] Porter, M.I. and Hills, D.A., 2002, "Inclined Edge Cracks in Compressive Stress Fields: Determination of Mode II Stress Intensity Factors", Journal of Strain Analysis, Vol.37, No: 2, pp.163-167.

[19] Choi, H.J., 2001, “The Problem for Bonded Half-Planes Containing a Crack at an Arbitrary Angle to the Graded Interfacial Zone", International Journal of Solids and Structure, Vol.38, pp.6559-6588.

[20] Guo L.C, Wang, Z.H. and Zhang, L., 2012, "A Fracture Mechanics Problem of a Functionally Graded Layered Structure with an Arbitrarily Oriented Crack Crossing the Interface”, Mechanics of Materials, Vol.46, pp.69-82.

[21] Delale, F., Bakirtas, I. and Erdogan, F., 1978, "The Problem of an Inclined Crack in an Orthotropic Strip", NASA-CR-157142, The National Aeronautics and Space Administration Grant No. NGR39-007-011. 
[22] Long, X. and Delale, F., 2004, "The General Problem for an Arbitrarily Oriented Crack in a FGM Layer", International Journal of Fracture, Vol.129, pp.221-238.

[23] Long, X. and Delale, F., 2005, "The Mixed Mode Crack Problem in an FGM Layer Bonded to Homogeneous Half-Plane”, International Journal of Solids and Structures, Vol.42, pp.3897-3917.

[24] Monfared, M.M. and Ayatollahi, M., 2016, "Multiple Crack Problems in Nonhomogeneous Orthotropic Planes under Mixed Mode Loading Conditions", Engineering Fracture Mechanics, Vol.155, pp.1-17.

[25] Jin, X. and Keer, L.M., 2006, "Solution of Multiple Edge Cracks in an Elastic Half Plane”, International Journal of Fracture, Vol.137, pp.121137.

[26] Yildirim, B., Kutlu, O. and Kadioglu, S., 2011, "Periodic Crack Problem for a Functionally Graded Half-Plane an Analytic Solution”, International Journal of Solids and Structures, Vol.48, pp.3020-3031.

[27] Dag, S., 1997, "Crack Problems in a Functionally Graded Layer under Thermal Stresses", M.S. Thesis, Department of Mechanical Engineering, Middle East Technical University, Ankara, Turkey.

[28] El-Borgi, S., Abdelmoula, R., Dag, S., and Lajnef, N., 2007, “A Surface Crack in a Graded Coating Bonded to a Homogeneous Substrate under General Loading Conditions", Journal of Mechanics of Materials and Structures, Vol.2, No:7, pp.1331-1353. 
[29] Dag, S., Apatay, T., Guler, M.A and Gulgec, M., 2012, “A Surface Crack in a Graded Coating Subjected to Sliding Frictional Contact", Engineering Fracture Mechanics, Vol.80 pp.72-91.

[30] Guo, B.K., Yan, H.H. and Zhang, L., 2016, "Cracking Angle of an Arbitrary Oriented Crack Embedded in a Strip under I-II Mixed Mode Loading”, International Journal of Material Science, Vol.6, and No: 1, pp.52-57.

[31] Yingzhi, L. and Hills, D.A., 1990, "Stress Intensity Factor Solutions for Kinked Surface Cracks”, Journal of Strain Analysis, Vol.25, No: 1, pp.2127.

[32] Lawn, B., 1993, Fracture of Brittle Solids, Cambridge University Press, Cambridge, UK.

[33] Hills, D.A. and Nowell, D., 1994, Mechanics Of Fretting Fatigue, Springer Science + Business Media Dorclrecht.

[34] Kimura, T. and Sato, K., 2003, "Simplified Method to Determine Contact Stress Distribution and Stress Intensity Factors in Fretting Fatigue", International Journal of Fatigue, Vol.23, pp.633-640.

[35] Elber, W., 1970, "Fatigue Crack Closure under Cyclic Tension", Eng. Fracture Mech., Vol.2, pp.37-45.

[36] Erdogan, F., Gupta, G.D. and Cook, T.S., 1973, "Numerical Solution of Singular Integral Equations", Mechanics of Fracture: Methods of Analysis and Solutions of Crack Problems, Sih, G.C., Springer Science+Business Media Dordrecht, pp.368-425. 
[37] Ozatag, A.C., 2003, "Contact Mechanics of a Graded Surface with Elastic Gradation in Lateral Direction", M.S. Thesis, Department of Mechanical Engineering, Middle East Technical University, Ankara, Turkey.

[38] Greenberg, M.D., 1998, Advanced Engineering Mathematics, Upper Saddle River, New Jersey, Prentice-Hall.

[39] Kreyszig, E., 2006, Advanced Engineering Mathematics, 9th Edition, John Wiley \& Sons, Inc.

[40] Hildebrand, F.B., 1962, Advanced Calculus For Applications, Englewood Cliffs, N.J., Prentice-Hall.

[41] Liu, R., Zhang, T., Wu, X. J., and Wang, C. H., 2004, "Effect of crack closure in a specially orthotropic cylindrical shell containing an axial or a circumferential crack", Engineering Fracture Mechanics, Vol.71(16-17), pp. 2493-2512.

[42] Beghini, M., Bertini, L. and Fontanari, V., 1999, "Stress Intensity Factors for an Inclined Edge Crack in a Semiplane", Engineering Fracture Mechanics, Vol.62, pp.607-613.

[43] Beghini, M., Bertini, L. and Fontanari, V., 2001, “A Weight Function Technique for Partially Closed Inclined Edge Cracks Analysis", International Journal of Fracture, Vol.112, pp. 57-68.

[44] Tricomi, F.G., 1951, "On the Finite Hilbert Transformation”, The Quarterly Journal of Mathematics Oxford Series, Vol.2, pp. 199-211.

[45] Abramowitz, M. and Stegun, I.A., 1972, Handbook of Mathematical Functions with Formulas, Graphs and Mathematical Tables, 10th Printing, U.S. Government Printing Office, Washington D.C. 


\section{APPENDIX A}

\section{EXPLICIT RELATIONS OF THE KERNELS OF CONTACT PROBLEM}

The expression of kernels $h_{13}(X, t)$ and $h_{23}(X, t)$ are provided as below.

$$
\begin{aligned}
& h_{13}(X, t)=\frac{C_{66}}{2} \sin ^{2}(\theta)\left[\left(\frac{R_{1}}{R_{2}}\right)+\left(\frac{-R_{1}}{R_{3}}\right)\right] \\
& +\frac{C_{66}}{2} \cos ^{2}(\theta)\left[\left(\frac{q_{2} R_{4} q_{1} \cos (\theta) X-\eta_{1} R_{4}(\sin (\theta) X+t)}{\left(d_{11} q_{1}^{2}+d_{12}\right) R_{2}}\right)\right. \\
& \left.+\left(\frac{-q_{1} R_{5} q_{2} \cos (\theta) X+\eta_{1} R_{5}(\sin (\theta) X+t)}{\left(d_{11} q_{2}^{2}+d_{12}\right) R_{3}}\right)\right] \\
& +\frac{C_{66}}{2} \sin (2 \theta)\left[\left(\frac{q_{1}^{2} \eta_{1} \cos (\theta) X+q_{1} q_{2}(\sin (\theta) X+t)}{R_{2}}\right.\right. \\
& \left.\left.+\frac{-q_{2}^{2} \eta_{1} \cos (\theta) X-q_{1} q_{2}(\sin (\theta) X+t)}{R_{3}}\right)\right] \\
& h_{23}(X, t)=\frac{C_{66}}{2} \sin (\theta) \cos (\theta)\left[\left(\frac{R_{1}}{R_{2}}\right)+\left(\frac{-R_{1}}{R_{3}}\right)\right] \\
& -\frac{C_{66}}{2} \sin (\theta) \cos (\theta)\left[\left(\frac{q_{2} R_{4} q_{1} \cos (\theta) X-\eta_{1} R_{4}(\sin (\theta) X+t)}{\left(d_{11} q_{1}^{2}+d_{12}\right) R_{2}}\right)\right. \\
& \left.+\left(\frac{-q_{1} R_{5} q_{2} \cos (\theta) X+\eta_{1} R_{5}(\sin (\theta) X+t)}{\left(d_{11} q_{2}^{2}+d_{12}\right) R_{3}}\right)\right] \\
& +\frac{C_{66}}{2} \cos (2 \theta)\left[\left(\frac{q_{1}^{2} \eta_{1} \cos (\theta) X+q_{1} q_{2}(\sin (\theta) X+t)}{R_{2}}\right.\right. \\
& \left.\left.+\frac{-q_{2}^{2} \eta_{1} \cos (\theta) X-q_{1} q_{2}(\sin (\theta) X+t)}{R_{3}}\right)\right] \\
& R_{1}=-q_{2} q_{1} \cos (\theta) X+\eta_{1}(\sin (\theta) X+t) \\
& R_{2}=\pi\left(-q_{2}+q_{1}\right)\left(q_{1}^{2} \cos (\theta)^{2} X^{2}+\sin (\theta)^{2} X^{2}+2 \sin (\theta) X t+t^{2}\right) \\
& R_{3}=\pi\left(-q_{2}+q_{1}\right)\left(q_{2}^{2} \cos (\theta)^{2} X^{2}+\sin (\theta)^{2} X^{2}+2 \sin (\theta) X t+t^{2}\right) \\
& R_{4}=\left(-d_{12} q_{1}^{2}-d_{12}^{2} q_{1}^{2}-d_{22}+d_{22} d_{11} q_{1}^{2}\right) \\
& R_{5}=\left(-d_{12} q_{2}^{2}-d_{12}^{2} q_{2}^{2}-d_{22}+d_{22} d_{11} q_{2}^{2}\right)
\end{aligned}
$$




\section{APPENDIX B \\ CLOSED FORM EXPRESSIONS FOR CAUCHY PRINCIPAL VALUE INTEGRALS}

Integrals related to Cauchy kernels are evaluated using the relation provided by Tricomi [44].

$$
\begin{aligned}
& \frac{1}{\pi} \int_{-1}^{1}(1-r)^{\psi_{2}}(1+r)^{\psi_{1}} P_{n}^{\left(\psi_{2}, \psi_{1}\right)}(r) \frac{d r}{s-r}= \\
& \quad-\cot \left(\pi \psi_{2}\right)(1-s)^{\psi_{2}}(1+s)^{\psi_{1}} P_{n}^{\left(\psi_{2}, \psi_{1}\right)}(s) \\
& +\frac{2^{\left(\psi_{2}+\psi_{1}\right)} \Gamma\left(\psi_{2}\right) \Gamma\left(n+\psi_{1}+1\right)}{\pi \Gamma\left(n+\psi_{1}+\psi_{2}+1\right)} F\left(n+1,-n-\psi_{2}-\psi_{1} ; 1-\psi_{2} ; \frac{1-s}{2}\right)
\end{aligned}
$$

In this relation, $F(\quad)$ designates Hypergeometric Function. For detailed information about the Hypergeometric Function, Abramowitz and Stegun [45] can be viewed. 